\title{
Binary asteroid population. 3. Secondary rotations and elongations
}

P. Pravec ${ }^{\text {a }}$, P. Scheirich ${ }^{\text {a }}$, P. Kušnirák ${ }^{\mathrm{a}}$, K. Hornoch ${ }^{\mathrm{a}}$, A. Galád ${ }^{\mathrm{a}, \mathrm{b}}$, S. P. Naidu ${ }^{\mathrm{c}}$, D. P. Pray ${ }^{\mathrm{d}}$, J. Világi ${ }^{\mathrm{b}}, \check{S}_{\text {S. Gajdoš }}^{\mathrm{b}}$, L. Kornoš ${ }^{b}$, Yu. N. Krugly ${ }^{e}$, W. R. Cooney ${ }^{f}, J_{\text {. Gross }}{ }^{\mathrm{f}}$, D. Terrell f,g, N. Gaftonyuk ${ }^{h}$, J. Pollock ${ }^{i}$, M. Husárik ${ }^{j}$, V. Chiorny ${ }^{\mathrm{e}}, \mathrm{R}$. D. Stephens ${ }^{\mathrm{k}}$, R. Durkee ${ }^{\ell}, \mathrm{V}$. Reddy ${ }^{\mathrm{m}}$, R. Dyvig ${ }^{n}$, J. Vraštil ${ }^{\mathrm{a}, \mathrm{o}}$, J. Žižka $^{\circ}$, S. Mottola ${ }^{\mathrm{p}}$, S. Hellmich ${ }^{\mathrm{p}}$, J. Oey ${ }^{\mathrm{q}}, \mathrm{V}$. Benishek ${ }^{\mathrm{r}}$, A. Kryszczyńska ${ }^{\mathrm{s}}$, D. Higgins ${ }^{\mathrm{t}}$, J. Ries ${ }^{\mathrm{u}}$, F. Marchis ${ }^{\mathrm{v}}$, M. Baek ${ }^{\mathrm{v}}$, B. Macomber ${ }^{\mathrm{w}}$, R. Inasaridze ${ }^{\mathrm{x}}, \mathrm{O}$. Kvaratskhelia ${ }^{\mathrm{x}}, \mathrm{V}$. Ayvazian ${ }^{\mathrm{x}}$, V. Rumyantsev ${ }^{\mathrm{y}}$, G. Masi ${ }^{\mathrm{z}, \mathrm{aa}}$, F. Colas ${ }^{\mathrm{ab}}$, J. Lecacheux ${ }^{\mathrm{ab}}$, R. Montaigut ${ }^{\text {ac }}$, A. Leroy ${ }^{\text {ac }}$, P. Brown ${ }^{\text {ad }}$, Z. Krzeminski ${ }^{\text {ad }}$, I. Molotov ${ }^{\text {ae }}$, D. Reichart ${ }^{\text {af }}$, J. Haislip ${ }^{\text {af }}$, A. LaCluyze ${ }^{\text {af }}$

a Astronomical Institute, Academy of Sciences of the Czech Republic, Fričova 1, CZ-25165 Ondřejov, Czech Republic

${ }^{\mathrm{b}}$ Modra Observatory, Department of Astronomy, Physics of the Earth, and Meteorology, FMPI UK, Bratislava SK-84248, Slovakia

${ }^{\mathrm{c}}$ Jet Propulsion Laboratory, California Institute of Technology, Pasadena, U.S.A.

d Sugarloaf Mountain Observatory, South Deerfield, MA, U.S.A.

${ }^{\mathrm{e}}$ Institute of Astronomy of Kharkiv National University, Sumska Str. 35, Kharkiv 61022, Ukraine

${ }^{\mathrm{f}}$ Sonoita Research Observatory, 77 Paint Trail, Sonoita, AZ 85637, U.S.A.

${ }^{\mathrm{g}}$ Deptartment of Space Studies, Southwest Research Institute, Boulder, CO 80302, U.S.A.

${ }^{\mathrm{h}}$ Crimean Astrophysical Observatory, Simeiz Department, Simeiz 98680, Crimea

${ }^{i}$ Physics and Astronomy Department, Appalachian State University, Boone, NC 28608, U.S.A.

${ }^{\mathrm{j}}$ Astronomical Institute of the Slovak Academy of Sciences, SK-05960 Tatranská Lomnica, Slovakia

${ }^{\mathrm{k}}$ Center for Solar System Studies, 9302 Pittsburgh Avenue, Suite 200, Rancho Cucamonga, CA 91730, U.S.A.

${ }^{\ell}$ Shed of Science Observatory, 5213 Washburn Ave. S, Minneapolis, MN 55410, U.S.A. 
${ }^{m}$ Planetary Science Institute, Tucson, Arizona 85719, U.S.A.

${ }^{\mathrm{n}}$ Badlands Observatory, 12 Ash Street, P.O. Box 37, Quinn, SD 57775, U.S.A.

${ }^{\circ}$ Institute of Astronomy, Faculty of Mathematics and Physics, Charles University,

Prague, V Holešovičkách 2, CZ-18000 Prague 8, Czech Republic

${ }^{\mathrm{p}}$ German Aerospace Center (DLR), Institute of Planetary Research, Rutherfordstr. 2, 12489 Berlin, Germany

${ }^{q}$ Blue Mountains Observatory, Leura, N.S.W., Australia

${ }^{\mathrm{r}}$ Belgrade Astronomical Observatory, Volgina 7, 11060 Belgrade 38, Serbia

${ }^{\mathrm{s}}$ Astronomical Observatory Institute, Faculty of Physics, Adam Mickiewicz University, Sloneczna 36, 60-286 Poznań, Poland

${ }^{\mathrm{t}}$ Hunters Hill Observatory, Ngunnawal, Canberra, Australia

${ }^{\mathrm{u}}$ The University of Texas at Austin, Astronomy Department/McDonald

Observatory, 1 University Station C1400, Austin, TX 78712-0259, U.S.A.

${ }^{\vee}$ Carl Sagan Center at the SETI Institute, 189 Bernado Av., Mountain View, CA 94043, U.S.A.

${ }^{\mathrm{w}}$ Department of Astronomy, University of California at Berkeley, Berkeley, CA 94720, U.S.A.

${ }^{\mathrm{x}}$ Kharadze Abastumani Astrophysical Observatory, Ilia State University, G. Tsereteli str. 3, Tbilisi 0162, Georgia

${ }^{y}$ Crimean Astrophysical Observatory, 98409 Nauchny, Crimea

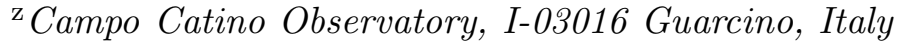

aa The Virtual Telescope Project, I-03023 Ceccano, Italy

${ }^{\mathrm{ab}}$ IMCCE-CNRS-Observatoire de Paris, 77 avenue Denfert Rochereau, 75014 Paris, France

${ }^{a c}$ OPERA Observatory, 33820 Saint Palais, France

ad Elginfield Observatory, Department of Physics $\&$ Astronomy, University of Western Ontario, London, Ontario N6A 3K\%, Canada


Miusskaya sq. 4, Moscow 125047, Russia

af Physics and Astronomy Department, University of North Carolina, Chapel Hill, NC 27514, U.S.A.

2015 December 8, revised version, submitted to Icarus 
Proposed running head: Secondaries of binary asteroids

Editorial correspondence to:

Dr. Petr Pravec

Astronomical Institute AS CR

Fričova 1

Ondřejov

CZ-25165

Czech Republic

Phone: 00420-323-620352

Fax: 00420-323-620263

E-mail address: ppravec@asu.cas.cz 


\begin{abstract}
We collected data on rotations and elongations of 46 secondaries of binary and triple systems among near-Earth, Mars-crossing and small main belt asteroids. 24 were found or are strongly suspected to be synchronous (in 1:1 spin-orbit resonance), and the other 22, generally on more distant and/or eccentric orbits, were found or are suggested to have asynchronous rotations. For 18 of the synchronous secondaries, we constrained their librational angles, finding that their long axes pointed to within $20^{\circ}$ of the primary on most epochs. The observed anti-correlation of secondary synchroneity with orbital eccentricity and the limited librational angles agree with the theories by (Ćuk, M., Nesvorný, D. [2010]. Icarus 207, 732-743) and (Naidu, S. P., Margot, J.-L. [2015]. Astron. J. 149, 80). A reason for the asynchronous secondaries being on wider orbits than synchronous ones may be longer tidal circularization time scales at larger semi-major axes. The asynchronous secondaries show relatively fast spins; their rotation periods are typically $<10 \mathrm{~h}$. An intriguing observation is a paucity chaotic secondary rotations; with an exception of (35107) $1991 \mathrm{VH}$, the secondary rotations are single-periodic with no signs of chaotic rotation and their periods are constant on timescales from weeks to years. The secondary equatorial elongations show an upper limit of $a_{2} / b_{2} \sim 1.5$. The lack of synchronous secondaries with greater elongations appears consistent, considering uncertainties of the axis ratio estimates, with the theory by Ćuk and Nesvorný that predicts large regions of chaotic rotation in the phase space for $a_{2} / b_{2} \gtrsim \sqrt{2}$. Alternatively, secondaries may not form or stay very elongated in gravitational (tidal) field of the primary. It could be due to the secondary fission mechanism suggested by (Jacobson, S. A., Scheeres, D. J. [2011]. Icarus 214, 161-178), as its efficiency is correlated with the secondary elongation. (Sharma, I. [2014]. Icarus 229, 278-294) found that rubble-pile satellites with $a_{2} / b_{2} \lesssim 1.5$ are more stable to finite structural perturbations than more elongated ones. It appears that more elongated secondaries, if they originally formed in spin fission of parent asteroid, are less likely to survive intact and they more frequently fail or fission.
\end{abstract}

Key words: Asteroids, satellites; Asteroids, rotation; Asteroids, dynamics; Near-Earth Objects; Photometry 


\section{Introduction}

Binary and multiple systems are frequent among asteroids smaller than about $15 \mathrm{~km}$ in (primary) diameter. The binary fraction in the population of nearEarth asteroids larger than $0.3 \mathrm{~km}$ was derived to be $15 \pm 4 \%$ (Pravec et al., 2006; a similar number was obtained by Margot et al., 2002, from a smaller sample), and our photometric survey for main belt asteroid binaries suggests a similar binary fraction among $D<15 \mathrm{~km}$ asteroids in the inner main belt. Our current knowledge of properties of asteroid binaries and theories of their formation and evolution are summarized in the review by Margot et al. (2015).

One of the key mechanisms determining evolution of binary asteroid systems is spin-orbit dynamics. It has been theoretically studied by several researchers, most recently by Naidu and Margot (2015). However, this and previous studies were limited by the scarcity of observational data on secondaries of asteroid binaries. In this paper, we have collected observational data on 46 secondaries of near-Earth, Mars-crossing and small main-belt asteroid systems. We have derived or constrained their spin rates and states and estimated their elongations. We have found certain trends in the secondary properties that provide constraints on the theories of evolution of the asteroid systems.

\section{Predictions from theories of satellite rotation}

A satellite formed in a general rotational state can be captured into spin-orbit resonance by the process of tidal despinning. Murray and Dermott (1999, Section 5) gave an overview of analytical theories of satellite rotation. They showed that an irregular satellite with the permanent quadrupole moment, i.e., permanent bulges or departures from sphericity, can be in a spin-orbit resonance with $p$ equal to an integer multiple of $+1 / 2$, with the rational $p$ defined by

$$
\gamma=\theta-p M
$$

where $\theta$ is an angle between the long axis of the satellite and a reference axis that lies in the orbit of the satellite around the primary and that is fixed in inertial frame (and which is chosen to be the line of apsides for a Keplerian orbit), and $M$ is the mean anomaly of the satellite orbiting the primary. The physical meaning of $\gamma$ is that it describes the orientation of the long axis of the satellite on passage of the satellite through pericenter, i.e., it is a stroboscopic angle that is evaluated when $M=0$. (The geometry is shown in Fig. 1.) They obtained the strength criterion

$$
\frac{\left|\left\langle N_{S}\right\rangle\right|}{\mathcal{C}}<\frac{1}{2} \omega_{0}^{2}
$$


where $\left|\left\langle N_{S}\right\rangle\right|$ is the mean tidal torque acting to change the spin of the satellite averaged over one orbital period, $\mathcal{C}$ is the satellite's moment of inertia around the spin axis, and $\omega_{0}$ is the libration frequency. It is

$$
\omega_{0}=n\left[3\left(\frac{\mathcal{B}-\mathcal{A}}{\mathcal{C}}\right)|H(p, e)|\right]^{\frac{1}{2}},
$$

where $n$ is the mean motion, $\mathcal{A}$ and $\mathcal{B}$ are the satellite's moments of inertia around the long and the intermediate principal axes, and $H(p, e)$ are factors dependent on $p$ and the satellite's orbital eccentricity $e$ (see Murray and Dermott, 1999, Eqs. 5.74 to 5.82) ${ }^{1}$. If the strength criterion (Eq. 2) is satisfied, then the mean torque due to the resonant interaction between the planet and the quadrupole moment of the satellite compensates for the mean tidal torque acting to change the spin of the satellite, $\langle\ddot{\gamma}\rangle=0$, and $\gamma$ librates about an equilibrium value $\gamma_{0}$. If the left term in Eq. 2 is much less than the right term, i.e., if the mean tidal torque is weak in comparison with the resonant torque, Murray and Dermott obtained that for $p=+1$ (i.e., 1:1 spin-orbit resonance) and $e<0.687, \gamma_{0} \approx 0$ or $\pi$ and the long axis of the satellite points towards the primary on passage of the satellite through pericenter.

For a satellite trapped in 1:1 spin-orbit resonance, the rotational motion of the satellite has short-period librations about the equilibrium configuration. This is because the full equation of motion contains short-period terms. Murray and Dermott (1999) derived that the amplitude of forced librations is

$$
\gamma_{\mathrm{A}}=\frac{2 \omega_{0}^{2} e}{\omega_{0}^{2}-n^{2}}
$$

If the forcing frequency $n$ is less than the natural frequency $\omega_{0}$, then the librations are in phase with the force. If $n>\omega_{0}$, then the librations and the force are $180^{\circ}$ out of phase. The resonance with $\omega_{0}=n$ occurs for $(\mathcal{B}-$ $\mathcal{A}) / \mathcal{C} \approx 1 / 3$, i.e., for the secondary equatorial axes ratio $a_{2} / b_{2} \approx \sqrt{2}$. Near the resonance, the secondary libration amplitude is high even for low-eccentricity orbits.

Murray and Dermott (1999) also touched the problem of asynchronous satellite rotation. Analysing surfaces of section of the satellite's rotational motion, they showed that chaotic motion occurs for elongated satellites on eccentric orbits. However, the purely analytical theory reaches its limits with this problem.

Ćuk and Nesvorný (2010) constructed a semi-analytical model of secondary rotation that is applicable for asteroid satellites on close orbits with the semimajor axis $a \lesssim 10 D_{1}$ (primary diameters). They found that satellites with the secondary-to-primary diameter ratio $D_{2} / D_{1} \sim 0.3$ (about a typical value for near-Earth and small main-belt asteroid binaries) on a very close orbit with $a=2 D_{1}$ and a moderate eccentricity $e=0.05$ can be trapped in the $1: 1$ synchronous rotation if its $(\mathcal{B}-\mathcal{A}) / \mathcal{C} \lesssim 0.35$ (cf. the value for the $\omega_{0}=n$

$\overline{1}$ The $H(p, e)$ are factors in the averaged equation of motion of the satellite's libration, see Murray and Dermott, 1999, Eq. 5.73. 
resonance above); more elongated secondaries are in chaotic rotation. The region of chaotic rotation extends to lower satellite elongations for eccentricities about 0.1 and higher. They concluded that asteroid secondaries should be vulnerable to chaotic rotation even for relatively low-eccentricity mutual orbits.

Naidu and Margot (2015) numerically simulated the coupled spin and orbital motions of two rigid bodies under the influence of their mutual gravitational potential. They broadly classified trajectories of asteroid satellites in rotationorbital phase space as resonant, non-resonant quasi-periodic, and chaotic. For synchronous satellites, they simulated librations in the coupled problem. They found that at high values of the secondary-to-primary mass ratio, the libration amplitudes are considerably smaller than the analytical estimate with Eq. 4, suggesting that spin-orbit coupling tends to damp libration amplitudes of large satellites. For smaller satellites, they found that libration amplitudes can reach observable values even for moderately elongated shapes. They further found that, unless the satellite's dynamically equivalent ellipsoid is very close to an oblate spheroid, there are large regions of the phase space where the spin state of an asynchronous satellite is chaotic. For secondary spin rates that are further away from a separatrix (the boundary of a resonance region of the phase space), the secondary is on a quasi-periodic trajectory. They made some interesting predictions and estimates on asteroid satellites potentially spending long times in chaotic rotation, delaying BYORP evolution that requires synchronous satellite rotation.

\section{Secondary rotation detection method}

A rotation of a spatially unresolved solid body can be observed as a timevarying flux of scattered light from an external source (e.g., the sun) illuminating the body. A time series of such photometric measurements, called "light curve", is analysed and, with an appropriate model, we derive or constrain a rotational period of the body and estimate its overall shape.

For a system consisting of two or more solid bodies, the integral light flux from the system comprises of components from the individual bodies. To the first order, the components are additive; second order effects of light scattered from one body illuminating another body can be neglected. A separation of the individual lightcurve components is straightforward when (1) eclipse/occultation events between the components are observed and (2) their rotational periods are not the same or commensurate. The observational and lightcurve decomposition techniques were described in Pravec et al. (2006).

A unique attribution of an individual rotational lightcurve component to a specific body in the asteroid system is based on a behavior of the lightcurve component during total eclipse/occultation events. If a given rotational lightcurve component disappears during the events, then we attribute it to the eclipsed/occulted body. However, if the rotational lightcurve component is present unchanged in the events, then it belongs to another body in the system. 
The photometric method provides a value for the mean rotational period, averaged over a few or several rotations of the body. Potential short-period variations in the spin rate are averaged out. It is in principle possible to reveal their presence from systematic residuals of the photometric data points from the mean rotational lightcurve, but this would require extensive and highquality photometric measurements covering the rotational cycle several times. We do not have such data available for most of our studied asteroid binaries; we derive the mean secondary rotational periods in this work.

For most of our studied systems where there is not a shape model of the secondary available, we estimate an equatorial elongation $\left(a_{2} / b_{2}\right)$ of the secondary from the observed amplitude of the secondary rotational lightcurve component $\left(A_{2}\right)$. We use the method described in Pravec and Harris (2007, Section 2.1). It assumes that the secondary's spin axis $\overrightarrow{c_{2}}$ is perpendicular to the orbital plane and so we observed the secondary close to the equator-on aspect at epochs where there occured mutual events between the system's components. If this assumption was not held, the estimated value would be a lower limit on $a_{2} / b_{2}$.

In our analyses of the secondary rotational lightcurves presented in Section 4.1, we use the assumption of bimodal lightcurve shape (i.e., we assume there are two pairs of maxima/minima per period). This assumption probably holds for all or a vast majority of the systems so that our interpretation of them being in 1:1 synchronous states is correct in most or all cases, for the following reasons. For secondaries with low-moderate elongations, there could possibly be an ambiguity in whether a secondary rotation period is the period derived from the rotational lightcurve analysis using the assumption of bimodal lightcurve shape (with two pairs of maxima/minima per period), or twice that. As shown in Harris et al. (2014), there may occur quadrumodal lightcurves with amplitudes (for single bodies) up to 0.38 mag. So, unless the derived $a_{2} / b_{2}$ is greater than 1.4 , it might happen that the secondary rotational period is actually twice the derived secondary lightcurve period. Thus, it could possibly be that some of the cases where we found 1:1 synchronous states with estimated low-moderate elongations could be actually 1:2 $(p=+1 / 2)$ resonance states with secondary shapes with approximately four-fold symmetry. ${ }^{2}$ However, such secondary shapes may be uncommon - at least, they are infrequent among single asteroids - and our assumption of bimodal secondary shape is probably held in most cases. Further, as shown by Murray and Dermott (1999, section 5.4), the rotation of a secondary trapped in a $p=+1 / 2$ resonance is anomalous in that on passage of the satellite through pericenter its long axis points in a direction perpendicular to the primary-secondary line. And, finally, for $\dot{\theta}=n / 2$ in the 1:2 resonance, it is not very likely to capture the secondary in an orientation with a lightcurve minimum coinciding with mutual events to within $10^{\circ}-20^{\circ}$, as we see for the synchronous secondaries at almost all epochs.

An orientation of the long axis of the secondary with respect to the line con-

$\overline{2}$ A figure with four-fold symmetry shows the same cross section when rotated by $90^{\circ}$. An example is a cube rotating around axis parallel to its edge. 
necting the centers of the primary and secondary can be estimated from a difference between the times of minima of the secondary rotational lightcurve and the times of mutual events. The minimum of a rotational lightcurve occurs approximately, but not exactly at a time when the body's longest principal axis points to the observer; a systematic offset can occur there due to irregular shape and phase effects. To estimate the magnitude of possible systematic offsets in the times of the secondary lightcurve minima due to these effects, we run following simulations. We generated synthetic rotational lightcurves for 1000 gaussian random spheres ${ }^{3}$ (Muinonen and Lagerros, 1998) rotating around the principal axis with the maximum moment of inertia and illuminated by a light source lying in the equatorial plane at phase angles $0^{\circ}$ and $20^{\circ}$. (These angles cover a typical range of phase angles in our observations of main belt asteroids.) For each simulated lightcurve, we computed a difference between the time of the lightcurve minimum and a time when the longest principal axis with the minimum moment of inertia coincides with the phase-angle bisector (PAB). ${ }^{4}$ In Fig. 2, we plot the time offsets for the 1000 simulated shapes, expressed as offsets in secondary's mean anomaly, against a ratio ${ }^{5}$ between the body's longest and intermediate axes, $a_{2} / b_{2}$. For secondary shapes with $a_{2} / b_{2}>1.2$, the systematic errors in the estimated mean anomaly offsets due to the irregular shape and phase effects are mostly $<10^{\circ}$ and almost always $<20^{\circ}$. We obtain that with the photometric method, we can resolve librational angles greater than $\sim 20^{\circ}$; smaller offsets between the secondary rotational lightcurve and the mutual events may be due to irregular secondary shapes and not secondary librations.

${ }^{3}$ Gaussian random spheres are described by spherical harmonics with Gaussian random coefficients, with standard deviations set so that their shape distribution mimics that of small solar system bodies.

4 The PAB is an approximation for an effective viewing direction as the minimum of a rotational lightcurve occurs close to a time when the body's long axis is closest to the PAB (Harris et al., 1984; see also Pravec et al., 2005a) and the mid-time of a mutual event (eclipse+occultation) between the primary and secondary of a binary asteroid is close to a time when the radius vector of the secondary is closest to the PAB.

5 We computed the axial ratios from cross-sections of the simulated bodies when viewed along the $a_{2}$ and $b_{2}$ axes, to mimic their derivation from rotational lightcurve amplitudes. 


\section{Observed secondary rotations}

The observational data for asteroid systems presented in this section are given in papers mentioned in the subsections on individual objects, or they are presented in Electronic Supplementary Information.

\subsection{Synchronous secondaries}

We have obtained 24 binary or triple asteroids with asynchronous primaries where our photometric or radar observations revealed or strongly suggested a synchronous secondary rotation. ${ }^{6}$ In Table 1 , we present the best estimated parameters for the binary systems. Uncertainties of and references for the individual values are available in the binary asteroid parameters tables at http://www.asu.cas.cz/ asteroid/binastdata.htm (update of the original tables from Pravec and Harris, 2007). The first three data columns in Table 1 are the primary diameter $D_{1}$, the secondary-to-primary diameter ratio $D_{2} / D_{1}$ and the ratio between the semimajor axis of the secondary orbit to the primary diameter $a / D_{1}$. The component diameter $D_{i}$ is the cross-section equivalent diameter (designated $D_{i, \mathrm{C}}$ in Scheirich et al., 2015a), i.e., the diameter of a sphere with the same cross section, of the $i$ th component of a given asteroid system at the equator-on aspect where the eclipse/occultation events were observed. A 3- $\sigma$ upper limit on the secondary orbit eccentricity is given in the fourth data column. In the next six columns, there are given the primary rotational period $P_{1}$, the orbital period $P_{\text {orb }}$, the secondary rotational period $P_{2}$, the amplitudes of the primary $\left(A_{1}\right)$ and secondary $\left(A_{2}\right)$ rotational lightcurves obtained after subtraction of the non-constant flux part of the other rotational lightcurve component, and the mean solar phase at which the amplitudes were measured. (The amplitude and solar phase values are omitted in a few cases where the equatorial axis ratios were derived from radar observations.) In the last two columns, we give values for the primary and secondary equatorial axis ratios $\left(a_{1} / b_{1}, a_{2} / b_{2}\right)$, estimated from the measured amplitudes of the lightcurve components using the method described in Pravec and Harris (2007; Section 2.1) or obtained from published shape models.

In Table 2, we report quantities describing observed rotational lightcurves of the synchronous secondaries at individual epochs when they could be precisely measured. In the second column, the epoch (close to the mid-time of given observational data series) of the measured secondary rotational lightcurve is given. In the next column, we give a value of the secondary rotational lightcurve period that we used for the fit of the Fourier series to the secondary lightcurve component (outside events). In some cases, this value differs slightly from the $P_{2}$ value given in Table 1 because of a presence of small synodic effect modifying the apparent period. In a few cases, the formal best fit value of the secondary period reported in Table 1 had a small but noticeable error and

6 In these semi-synchronous systems, the primary rotates with a period different
from the orbital period of the secondary, while the secondary rotates synchronously. 
we used a value closer to the secondary's orbital period for the Fourier series fit representing the secondary rotational lightcurve at the given epoch. In the fourth column, we report which harmonics appeared significant and were fitted; other Fourier series orders were omitted in the fit. In the next column, the mean solar phase of the observations is given. An amplitude of the fitted Fourier series representing the secondary rotational lightcurve obtained after subtracting the non-constant flux part of the primary rotational lightcurve is given in the column $A_{2}$. In the next three columns, there are given a phase of the event (for the mid-time between the first and the last contact), a phase of the minimum of the 2 nd harmonic of the Fourier series fitted to the secondary rotational lightcurve, and a difference between these two phases. In the one before the last column, the phase difference is multiplied by $360^{\circ}$ to give an offset in mean anomaly between the secondary rotational lightcurve minimum and the event; this is an estimate for the librational angle. In the last column, the figure with a plot of the secondary rotational lightcurve component data and the fitted Fourier series is indicated.

In Fig. 3 for (2121) Sevastopol and analogous figures for the other binaries with synchronous secondaries, the data of the secondary rotational lightcurve component and superimposed eclipse/occultation events are plotted. The data were reduced to the unit geo- and heliocentric distances and to a reference solar phase, and the variable flux part of the primary rotational lightcurve was subtracted, using the decomposition method described in Pravec et al. (2006). The red curve is the fitted Fourier series representing the secondary rotational lightcurve for the given epoch. In Fig. 4 for (2121) Sevastopol and analogous figures for the other synchronous binaries, the logarithm of reduced $\chi^{2}$ (i.e., $\chi^{2}$ per degree of freedom of fit) vs $P_{2}$ for the Fourier series fit to the data for one of the epochs for a given binary is plotted. In the three cases (66063) 1998 RO1, (85938) 1999 DJ4 and (175706) 1996 FG3, the observers did not estimate errors for their individual photometric measurements and thus we could not compute reduced $\chi^{2}$; we plot the logarithm of a sum of square residuals of the fit on an arbitrary flux scale on the y-axis in Figs. 21, 25 and 28.

\subsection{1 (2121) Sevastopol}

The binary nature of this main belt asteroid was discovered by Higgins et al. (2010). We have observed it in three apparitions 2010, 2012 and 2013. A model of the binary will be presented in Scheirich et al. (in preparation). A secondary rotational lightcurve was resolved in all the three apparitions, see Fig. 3. It is in 1:1 synchronous state, see Fig. 4. The minima of the secondary lightcurves were aligned with the eclipse/occultation events to within $10^{\circ}$. Other resonance states (such as a 3:2 spin-orbit resonance) are entirely inconsistent with the data and they are ruled out.

\subsection{2 (2131) Mayall}

The binary character of this Mars-crossing asteroid was discovered by Warner et al. (2009). We have observed it in three apparitions 2009, 2011 and 2013. 
A model of the binary will be presented in Scheirich et al. (in preparation). A secondary rotational lightcurve was well resolved in the discovery apparition 2009 where the most abundant data were obtained (Fig. 5); in the following two apparitions, we saw signs of the secondary rotation as well, consistent with the 2009 data. It is in 1:1 synchronous state, see Fig. 6. The minima of the secondary lightcurve were aligned with the events to within a few degrees.

\section{1 .3 (5477) Holmes}

The binary nature of this Hungaria asteroid was discovered by Warner et al. (2005a). Observations from the first two apparitions 2005 and 2007 were published in Pravec et al. (2012), and we have obtained additional data in two more apparitions 2012 and 2013. A model of the binary will be presented in Scheirich et al. (in preparation). A secondary rotational lightcurve was well resolved in the three post-discovery apparitions, see Fig. 7; in the discovery apparition, we saw signs of the secondary rotation as well. It is in 1:1 synchronous state (Fig. 8). The minima of the secondary lightcurves were aligned with the events to $\leq 13^{\circ}$. A 3:2 spin-orbit resonance (period $16.27 \mathrm{~h}$ ) appears unlikely, as it gives a poorer fit and the two best observational runs show systematic residuals for that period.

\section{1 .4 (5481) Kiuchi}

The binary nature of this main belt asteroid was discovered by Kušnirák et al. (2008). We have observed it in three apparitions 2008, 2013 and 2015. A model of the binary will be presented in Scheirich et al. (in preparation). A secondary rotational lightcurve was resolved in all the three apparitions, see Figs. 9 and 10. The data show it to be in 1:1 synchronous state, see Fig. 11. The minima of the secondary lightcurves were aligned with the eclipse/occultation events to within $10^{\circ}$ on four of the five epochs, but there was a significant mis-alignment by $\sim 50^{\circ}$ shown by the observations on four nights 2015-0214 to 18. They are observations from a single station (PROMPT); a possible significant librational amplitude will need to be confirmed with more thorough observations in the future.

\section{1 .5 (5905) Johnson}

The binary nature of this Hungaria asteroid was discovered by Warner et al. (2005b). Observations from the first two apparitions 2005 and 2008 were published in Pravec et al. (2012), and we have obtained additional data in two more apparitions 2011 and 2013. A model of the binary will be presented in Scheirich et al. (in preparation). A secondary rotational lightcurve was well resolved in all but the 2008 apparition where only low quality data were obtained; Figs. 12 to 14. It is in 1:1 synchronous state (Fig. 15). The minima of the secondary lightcurves were aligned with the events to within $20^{\circ}$. 


\subsection{6 (7088) Ishtar}

The binary nature of this Amor asteroid was discovered by Reddy et al. (2006); it was not observed since the discovery apparition. The observational data are presented in Electronic Supplementary Information. A secondary rotational lightcurve was clearly resolved (Fig. 16), indicating that it is in 1:1 synchronous state (Fig. 17). The minima of the secondary lightcurve were aligned with the events to about $10^{\circ}$.

\subsection{7 (44620) $1999 R S 43$}

We have discovered the binary nature of this main belt asteroid with our observations from 2014-03-26 to 04-05. The observational data are presented in Electronic Supplementary Information. These observations were run as a part of our photometric project "NEOSource" within its task to study binary and multiple systems among asteroid pairs (i.e., pairs of asteroids that are in highly similar heliocentric orbits, see Vokrouhlický and Nesvorný 2008, Pravec et al. 2010). Asteroid (44620) was selected as a target, as we found it to be paired with asteroid (295745) 2008 UH98 based on a similarity of their orbits. Our backward integrations of their orbits indicate that the pair components separated approximately $800 \mathrm{kyr}$ ago. A secondary rotational lightcurve was resolved (Fig. 18), indicating that it is in 1:1 synchronous state (Fig. 19). The minima of the secondary lightcurve were aligned with the events to a few degrees. Other resonance states (such as a 3:2 spin-orbit resonance) are inconsistent with the data.

\section{1 .8 (66063) 1998 RO1}

The binary nature of this near-Earth asteroid was discovered by Pravec et al. (2003). A model of the binary was published in Scheirich and Pravec (2009). A secondary rotational lightcurve was resolved in both 2003 and 2004 apparitions, see Fig. 20. It is in 1:1 synchronous state, see Fig. 21. The minima of the secondary lightcurves were aligned with the eclipse/occultation events to within $10^{\circ}$.

\section{1 .9 (66391) $1999 \mathrm{KW}_{4}$}

From their modeling of the radar observations of this near-Earth binary asteroid taken in 2001, Ostro et al. (2006) constrained the secondary rotational period between 17.3 and $17.5 \mathrm{~h}$, but they "could not discriminate between specific values in that interval." They further noted that in their modeling of the secondary, their "synthesized images ... could not fit image fine structure as well as with [the primary]." Their further experiments were "suggestive of [the secondary's] rotation not being exactly synchronous" and that "[the secondary] may exhibit sizable librations in longitude." 
The binary nature of this Hungaria asteroid was discovered by Warner et al. (2005c). Observations from the first two apparitions 2005 and 2008-2009 were published in Pravec et al. (2012), and we have obtained additional data in the apparition 2013. A model of the binary will be presented in Scheirich et al. (in preparation). A secondary rotational lightcurve was well resolved in the first and the third apparitions, see Fig. 22; in the second apparition, we saw signs of the secondary rotation as well. It is in 1:1 synchronous state (Fig. 23). The minima of the secondary lightcurves were aligned with the events to about $10^{\circ}$.

\section{1 .11 (85938) 1999 DJ4}

The binary nature of this Apollo asteroid was discovered by Pravec et al. (2004); it was not observed since the discovery apparition. The observational data were presented in Pravec et al. (2006). A secondary rotational lightcurve was clearly resolved (Fig. 24), indicating that it is in 1:1 synchronous state (Fig. 25). The minima of the secondary lightcurve were aligned with the events to about $11^{\circ}$.

\subsubsection{2 (175706) 1996 FG3}

The binary nature of this Apollo asteroid was discovered by Pravec et al. (1998a). It was observed in as many as six apparitions between 1996 and 2013. A model of the binary was published in Scheirich et al. (2015a). A secondary rotational lightcurve was resolved in four of the six apparitions, see Figs. 26 and 27; in the other two apparitions, there were obtained limited/low quality data that did not allow a detection of the secondary rotation. It is in 1:1 synchronous state, see Fig. 28. (The two additional minima of the noise spectrum around 12 and 24 hours are aliases with Earth's rotation.) The minima of the secondary lightcurves were aligned with the eclipse/occultation events to within $20^{\circ}$.

\subsubsection{3 (185851) 2000 DP107}

This Apollo asteroid was discovered to be binary from radar observations (Ostro et al., 2000; Margot et al., 2000, 2002). A rotation of the secondary was found to be synchronous by Naidu et al. (2015).

\section{1 .14 (399774) $2005 \mathrm{NB}$}

The binary nature of this Apollo asteroid was discovered by Shepard et al. (2008). We took follow-up observations of the binary shortly after the radar discovery. The observational data are presented in Electronic Supplementary Information. We resolved a secondary rotational lightcurve, see Fig. 29. It is in 1:1 synchronous state, see Fig. 30. The minima of the secondary lightcurve were aligned with the events to within $10^{\circ}$. 


\subsubsection{5 (3309) Brorfelde}

The binary nature of this Hungaria asteroid was discovered by Warner et al. (2005d). Observations from the first three apparitions 2005, 2009 and 2010 were published in Pravec et al. (2012), and we have obtained additional data in two more apparitions 2012 and 2014. A model of the binary will be presented in Scheirich et al. (in preparation). A secondary rotational lightcurve was resolved in the highest quality data taken on two epochs in 2010 as well as in additional data in 2012, see Fig. 31. It is likely in 1:1 synchronous state, see Fig. 32. Though the amplitude is low, the data from the three epochs agree well and other resonance states do not appear likely. The minima of the secondary lightcurves were aligned with the eclipse/occultation events to within $10^{\circ}$.

\subsubsection{6 (9260) Edwardolson}

The binary nature of this main belt asteroid was discovered by Jakubík et al. (2005). We have obtained observations in the apparitions 2005 and 2012. The observational data are presented in Electronic Supplementary Information. A secondary rotational lightcurve was resolved in both apparitions, see Fig. 33. Though the amplitude is low, it is clearly seen in several long and good quality runs. It is likely in 1:1 synchronous state, see Fig. 34. A 3:2 spin-orbit resonance does not appear likely as the two highest quality runs in 2012 show systematic residuals for that period. The minima of the secondary lightcurves were aligned with the eclipse/occultation events to within $10^{\circ}$.

\section{$4.1 .17 \quad(17260) 2000 \mathrm{JQ58}$}

The binary nature of this main belt asteroid was discovered by Higgins et al. (2006). Observations from the first two apparitions 2006 and 2009 were published in Pravec et al. (2012), and we have obtained additional data in the apparition 2011. A model of the binary will be presented in Scheirich et al. (in preparation). A secondary rotational lightcurve was well resolved in the first and the third apparitions, see Fig. 35; in the second apparition, lower quality data were obtained that did not allow a detection of the secondary rotation. It is likely in 1:1 synchronous state (Fig. 36); other resonance states do not appear likely. The minima of the secondary lightcurves were aligned with the eclipse/occultation events to within $10^{\circ}$.

\section{1 .18 (80218) 1999 VO123}

We have discovered the binary nature of this main belt asteroid with our observations from 2012-10-14 to 23, and we took follow-up observations from 2014-03-02 to 08. The observational data are presented in Electronic Supplementary Information. These observations were run as a part of our photometric project "NEOSource" within its task to study binary and multiple systems among asteroid pairs (i.e., pairs of asteroids that are in highly similar heliocentric orbits, see Vokrouhlický and Nesvorný 2008, Pravec et al. 2010). 
Asteroid (80218) was selected as a target, as we found it to be paired with asteroid (213471) 2002 ES90 based on a similarity of their orbits. Our backward integrations of their orbits indicate that the pair components separated $114 \pm 20$ kyr ago. A secondary rotational lightcurve was resolved in both apparitions, see Fig. 37. The data from the better covered 2012 apparition show it to be likely in 1:1 synchronous state (Fig. 38). We obtained a poorer fit for 3:2 spin-orbit resonance, but we will want to rule out it entirely with more observations in the future. The minima of the secondary lightcurve were aligned with the eclipse/occultation events to within $10^{\circ}$ in the first apparition, but a significant mis-alignment by $\sim 40^{\circ}$ was shown by the observations on the five nights in 2014. A possible significant librational amplitude will have to be confirmed with more observations in the future.

\section{1 .19 (1453) Fennia}

The binary nature of this Hungaria asteroid was discovered by Warner et al. (2007). Observations from the first three apparitions 2007, 2009 and 2011 were published in Pravec et al. (2012), and we have obtained additional data in the apparition 2012. A model of the binary will be presented in Scheirich et al. (in preparation). A secondary rotational lightcurve was resolved in the highest quality data in 2011 and 2012, see Fig. 39. The amplitude is low, but the data are consistent with 1:1 synchronous state. In particular, a 3:2 spin-orbit resonance does not fit. Though a confirmation is needed with very high quality observations in the future, we suggest that it is a synchronous binary. The minima of the secondary lightcurves appear aligned with the eclipse/occultation events to within $15^{\circ}$.

\subsubsection{0 (5407) 1992 AX}

This Mars crosser was suspected to be binary by Pravec et al. (2000, 2006) based on their observations in 1997. We have taken follow-up observations in apparitions 2007 and 2012, confirming the asteroid's binary nature. A model of the binary will be presented in Scheirich et al. (in preparation). A secondary rotational lightcurve was resolved in all the three apparitions, see Figs. 40 and 41. The amplitude is low, but the data are consistent with 1:1 synchronous state. Though a confirmation is needed with high quality observations in the future, we suggest that it is a synchronous binary. The minima of the secondary lightcurves appear aligned with the events to within $20^{\circ}$.

\subsubsection{1 (8306) Shoko}

The binary nature of this main belt asteroid was discovered by Pravec et al. (2013). Like asteroids (44620) and (80218) above, it was observed in our photometric project "NEOSource" within its task to study binary and multiple systems among asteroid pairs. Asteroid (8306) is paired with 2011 SR158, they separated about $500 \mathrm{kyr}$ ago, see Pravec et al. (2013) for details. Our analysis of the 2013 observations showed a presence of as many as three periods outside 
mutual events: the primary period $P_{1}=3.3503 \pm 0.0002 \mathrm{~h}$ and two secondary periods. One of the secondary periods coincides with the orbital period $36.2 \mathrm{~h}$ of the satellite that was in the eclipse/occultation geometry in 2013. This suggests that the satellite is in 1:1 synchronous state. The additional brightness variation suggests a presence of a third body in the system. Its rotational period is suggested to be about 61 hours, but this needs to be confirmed with future observations. Anyway, (8306) Shoko may be another case of a triple asteroid with synchronous inner satellite and asynchronous outer satellite; see Section 4.2 below.

\subsubsection{2 (16635) 1993 QO}

The binary nature of this main belt asteroid was discovered by Pray et al. (2007a). We have obtained follow-up observations from 2014-08-20 to 10-01. In the discovery apparition 2007, the data revealed two rotational components with superimposed mutual events. The two rotational components had periods of $2.2083 \pm 0.0002 \mathrm{~h}$ and $7.622 \pm 0.002 \mathrm{~h}$ with apparent amplitudes of 0.17 and $0.05 \mathrm{mag}$, respectively. Like in the cases of (1830) Pogson, (2006) Polonskaya and (2577) Litva published in Pravec et al. (2012) and given in Section 4.2.2 below, both rotational components were present at all orbital phases including mutual events, with unchanged shape in the event. The fact that the second rotational component did not disappear in mutual events indicated that it was not a rotation of the eclipsing secondary. We considered that it might rather belong to a third body in the system. This suggestion has been strengthened with the follow-up observations in 2014 that showed a third rotational lightcurve component outside mutual events with an amplitude of 0.03 mag and a period coinciding with the orbital period of $32.25 \mathrm{~h}$, suggesting that it is a rotational lightcurve of the eclipsing satellite, which is in 1:1 synchronous state. The two rotational lightcurves seen in 2007 have also been detected in 2014 and they had periods of $2.2083 \pm 0.0002 \mathrm{~h}$ and $7.624 \pm 0.002 \mathrm{~h}$ and amplitudes of 0.20 and $0.04 \mathrm{mag}$, respectively. So, the data suggest that (16635) is another case of a triple asteroid with one synchronous and one asynchronous satellite; see Section 4.2.2 below.

\subsubsection{3 (136617) $1994 C C$}

This triple near-Earth asteroid system consists of a larger inner secondary on a low-eccentricity orbit and a smaller outer secondary on an orbit with eccentricity of $0.192 \pm 0.014$ (Fang et al., 2011). Brozović et al. (2011) estimated rotational periods of the secondaries from their mean bandwidths and estimated diameters, assuming that their sub-radar latitudes were close to equatorial, obtaining $P_{2}=26 \pm 12 \mathrm{~h}$ and $P_{3}=14 \pm 7 \mathrm{~h}$, respectively. The estimated rotational period of the inner secondary is similar to its orbital period of $29.8 \pm 0.8 \mathrm{~h}$ (Fang et al., 2011), suggesting that it is in 1:1 synchronous state; the outer secondary appears non-synchronous. 
A secondary rotation period of this binary near-Earth asteroid was estimated to be $15_{-7}^{+11} \mathrm{~h}$ (Shepard et al., 2006).

\subsection{Asynchronous secondaries}

Unlike the asteroid systems with synchronous secondaries presented above, systems with asynchronous satellites are mostly less well described. It is largely because they are observationally more demanding objects, especially for photometric observations, as they are often found on wider orbits or in triple systems. In this section, we present data for 22 asteroid systems where the observations revealed or suggested asynchronous secondary rotations. In Table 3, we give estimated parameters for the systems. The presented quantities are the same as in Table 1, except following: The index $s$ gives whether the asynchronous satellite is the second or the third (known) body in the system, counted from the center of mass of the system; the outer satellite in a triple system has $s=3$. Several less definitely determined values are given in parentheses; see details in the text of this section. Parameters of (synchronous) inner satellites of triple systems with asynchronous outer satellites are mentioned in the text or they can be found in the references.

\subsubsection{Close binary systems with asynchronous satellites}

We know five close ${ }^{7}$ binary systems among near-Earth asteroids where the radar or lightcurve observations revealed or suggested a non-synchronous secondary rotation.

The binary nature of (35107) 1991 VH was discovered by Pravec et al. (1998b) from their observations taken in 1997. The observations in the discovery apparition were of limited quality and quantity for detecting a rotational lightcurve of the satellite, but we obtained good follow-up observations in February 2003 when we resolved a second rotational lightcurve component with an apparent period of $12.836 \mathrm{~h}$ (error $<0.01 \mathrm{~h}$ ), see Pravec et al. (2006). In Fig. 42, we show a noise spectrum for the second rotational lightcurve component in the 2003 data (which is shown in Pravec et al., 2006, Fig. 12); the period of $12.836 \mathrm{~h}$ provides the best fit for a bimodal lightcurve with two pairs of maxima and minima per cycle. We strongly suspect that this second lightcurve component was due to a rotation of the satellite; the period of $12.836 \mathrm{~h}$ could be a mean period of the secondary during the observational interval 200302-02 to 28. It is substantially shorter than the satellite's orbital period of $32.7 \mathrm{~h}$, suggesting a non-synchronous rotational state of the secondary. Its apparent amplitude of $0.06 \mathrm{mag}$ in the combined primary+secondary data

$\overline{7 \text { Close }}$ asteroid binaries are systems where the satellite orbits the primary at a distance small enough so that it could be efficiently synchronized by tides from the primary. From theory and observations, we estimate that they are systems with the relative semi-major axis $a / D_{1}$ less than about $6-7$, typically. 
converts to $a_{2} / b_{2}=1.33 \pm 0.10$, suggesting a moderate elongation of the secondary. Our additional observations obtained between 2008-06-24 and 07-08 showed a secondary rotational brightness variation with an amplitude about 0.06 mag again, but with a different period than the $12.836 \mathrm{~h}$ seen in February 2003. A limited amount of data obtained during the short June-July nights on our northern hemisphere station hampered to obtain a unique estimate for a mean secondary period during the 2008 observational interval, but there were several possible values between 7 and 29 hours that all differed from $12.836 \mathrm{~h}$. It appears that the secondary period is not constant. It is notable that the eccentricity of the satellite's orbit is not zero; Pravec et al. (2006) derived $e=0.05 \pm 0.02$ (3- $\sigma$ error) from the 1997 observations, and Naidu et al. (2012) reported an eccentricity of 0.05 from their radar observations taken in August 2008. The radar observations also confirmed the non-synchronous secondary rotation, as they wrote "no acceptable fit to the sequence of secondary images has been found under the assumption of synchronous spin."

Brozović et al. (2011) report that the secondary's delay-Doppler dispersion of radar observations of the near-Earth binary asteroid (162000) 1990 OS by S. Ostro and collaborators suggested a secondary rotational period $\leq 8 \mathrm{~h}$, substantially shorter than the orbital period of $21 \pm 3 \mathrm{~h}$ (Ostro et al., 2003).

For (164121) 2003 YT1, Nolan et al. (2004) estimated a secondary rotational period $\leq 6 \mathrm{~h}$, which is much shorter than the orbital period of $36.7 \pm 1.8 \mathrm{~h}$ estimated by Brooks (2006). They also noted that the secondary's orbit was eccentric, though they did not report a quantitative estimate.

Taylor et al. (2008) report that their radar observations of the near-Earth binary asteroid (311066) 2004 DC suggested a secondary rotational period of $7 \mathrm{~h}$, substantially shorter than the orbital period of $\sim 23 \mathrm{~h}$. They also report an eccentricity of 0.24 .

For (363027) 1998 ST27, Benner et al. (2003) constrained the secondary rotational period to be $<6 \mathrm{~h}$. They also estimated the eccentricity $\geq 0.3$ and suggested "that the orbital period is several days".

\subsubsection{Triple systems with asynchronous outer satellites}

We know two triple near-Earth asteroids with asynchronous outer secondaries, (136617) 1994 CC and (153591) 2001 SN263, and we have five candidate triple main-belt asteroids with similar characteristics.

For data on (136617) 1994 CC, see Sect. 4.1.23. The system (153591) 2001 SN263 consists of a smaller inner secondary and a larger outer secondary, both on low-eccentricity orbits (Fang et al., 2011). Becker et al. (2015) derived a rotational period of the outer secondary to be $13.43 \pm 0.01 \mathrm{~h}$, much shorter than its orbital period of $149.4 \pm 2.3 \mathrm{~h}$ obtained by Fang et al. (2011); a rotational state of the inner satellite has not been established. They found a moderately elongated shape for the outer secondary with $a_{3} / b_{3} \approx 1.3$.

Pravec et al. (2012) published their observations of secondary rotational light- 
curves in three systems (1830) Pogson, (2006) Polonskaya and (2577) Litva. In each of the three cases, the secondary rotational lightcurve has a period much shorter than the orbital period of the observed close eclipsing/occulting secondary that has shown mutual events with the primary. The character of the secondary rotational lightcurve, namely the fact that it does not disappear in the events, indicates that it is not a rotation of the eclipsing secondary. Rather, they suspected that it belongs to a third body (second satellite) in the system. This has been confirmed with adaptive optics observations of a distant satellite in the system of (2577) Litva by Merline et al. (2013). They obtained the bestfit estimate for its orbital period of 214 days, but they could not rule out a period of half that. We suggest that the observed secondary rotational period of $5.6818 \pm 0.0004 \mathrm{~h}$ (Pravec et al., 2012) belongs to this distant satellite. The amplitude of its rotational lightcurve component was measured to be 0.06 $0.09 \mathrm{mag}$ at solar phase $11-30^{\circ}$ that converts to $a_{3} / b_{3}=1.85 \pm 0.47$, using $D_{3} / D_{1}=0.32$ suggested by its measured brightness difference from the close inner pair reported by Merline et al. (2013). No observational constraint on rotation of the eclipsing close satellite has been obtained.

The other two systems published in Pravec et al. (2012), Pogson and Polonskaya, show characteristics very similar to Litva. We suspect that their observed secondary rotational periods $3.2626 \pm 0.0004 \mathrm{~h}$ and $6.6593 \pm 0.0004 \mathrm{~h}$, respectively (Pravec et al., 2012), belong to third bodies (second satellites) in the systems as well. Like in the case of Litva, the asynchronous second satellites of Pogson and Polonskaya likely revolve the primaries on wider orbits than the close secondaries that showed eclipse/occultation events. This is supported by the fact that despite our extensive follow-up observations of these two systems - we have already observed Pogson in six and Polonskaya in four apparitions covering a broad range of aspects-, we have detected no events from the second satellites, which would be unlikely if the satellites were on close orbits. (New orbital models of the eclipsing close secondaries of these three systems will be presented in Scheirich et al., in preparation).

Another asteroid system of this type is (16635) 1993 QO, see Section 4.1.22. The case of (8306) Shoko described in Section 4.1.21 may be a similar system as well, though the rotational period of its suspect outer satellite is relatively long $(61 \mathrm{~h})$. Unlike in Pogson, Polonskaya and Litva, the rotations of the eclipsing close secondaries of (16635) and Shoko have been constrained: they appear synchronous.

\subsection{3 (Semi-)wide systems with asynchronous satellites}

We know three binary systems and one candidate triple system with asynchronous satellites on semi-wide orbits with orbital periods between 100 and 1000 hours.

Merline et al. (2003a) discovered a satellite of (1509) Esclangona. They reported a brightness difference between the secondary and the primary of about $2.4 \mathrm{mag}$, corresponding to $D_{2} / D_{1} \approx 0.33$. In Merline et al. (2003b), they report that "the companion appears to orbit at least 23 primary radii 
from [the primary]". Warner et al. (2010) has observed a secondary rotational lightcurve with $P_{2}=6.6422 \pm 0.0004 \mathrm{~h}$ and an amplitude (in the combined primary+secondary lightcurve) of $0.04 \mathrm{mag}$ at solar phase $23^{\circ}$ that converts to $a_{2} / b_{2}=1.29 \pm 0.11$.

The main belt asteroid (2623) Zech was found to have a satellite on a semiwide orbit with $P_{\text {orb }}=117.2 \pm 0.3 \mathrm{~h}$ (Pray et al., 2014). The observational data are presented in Electronic Supplementary Information. They observed two rotational lightcurve components with periods $P_{1}=2.7401 \pm 0.0002 \mathrm{~h}$ and $P_{2}=18.718 \pm 0.008 \mathrm{~h}$. Unlike in the cases presented in Section 4.2.2 above, the secondary rotational lightcurve disappeared in mutual events, indicating that it belongs to the observed eclipsing satellite.

The main belt asteroid (32039) $2000 \mathrm{JO} 23$ was found to have a large satellite on a relatively wide orbit with $P_{\text {orb }}=360 \mathrm{~h}$ and a lower limit on $D_{2} / D_{1}$ of 0.58 (Pray et al., 2007b). They observed two rotational lightcurve components, one with a period of $3.2990 \pm 0.0002$ or $6.5979 \pm 0.0009 \mathrm{~h}$ and the other with a period of $11.099 \pm 0.003 \mathrm{~h}$. Our follow-up observations obtained during 201411-15 to 12-20 revealed the two rotational lightcurves again and they resolved the period ambiguity: it is $P_{1}=6.598 \mathrm{~h}$. Mutual events were not captured in the 2014 observations. The observational data are presented in Electronic Supplementary Information. The observations taken in the mutual events in 2007 do not resolve it uniquely whether the secondary rotational lightcurve component belongs to the observed eclipsing satellite, or if it might be of a possible third body in the system; in any case, it suggests a presence of a non-synchronous secondary.

The main belt asteroid (1717) Arlon was found to have a large satellite on a semi-wide orbit with $P_{\text {orb }}=117 \mathrm{~h}$ and a lower limit on $D_{2} / D_{1}$ of 0.5 (Cooney et al., 2006a,b). They observed two rotational lightcurve components with periods of 5.148 and $18.23 \mathrm{~h}$ and amplitudes of 0.08 and 0.13 mag, respectively. Our follow-up observations obtained in 2008, 2011, 2013 and 2014 revealed a presence of the two rotational lightcurves in all the apparitions, and the mutual events that were detected in February-April 2006 were observed in August-September 2014 again. An orbital model of the eclipsing satellite will be presented in Scheirich et al. (in preparation). Like in the systems presented in Section 4.2.2 above, none of the two rotational lightcurves disappeared in the mutual events so none of them belongs to the eclipsing semi-distant satellite; rather, it suggests a presence of a third body (second satellite) in the system. Though its orbit has not been estimated as we did not observe its eclipse/occultation events, it is unlikely that it could be on a close orbit with an orbital period as short as $18.23 \mathrm{~h}$, as in such case we would almost certainly observe its events in at least some of the five apparitions that covered a broad range of aspects. Thus, we suspect that the third body (second satellite) is asynchronous. A confirmation and determination of its orbit will be needed in the future. 


\subsubsection{Cases with two rotational lightcurves}

Over the last ten years (from 2005 to mid-2015) we and collaborating observers have found six main-belt asteroids that showed two rotational lightcurve components, but where we did not capture eclipse/occultation events. They are (2486) Metsahovi, (3982) Kastel', (5474) Gingasen, (11217) 1999 JC4, (13123) Tyson and (114319) 2002 XD58 (Pikler et al., 2007; Pravec et al., 2005b; Higgins et al., 2008; Warner, 2014; this work; Pray et al., 2005). Their periods and amplitudes are given in Table 3, and their observational data (except for 11217 which was published in Warner, 2014) are presented in Electronic Supplementary Information. We suspect that they are binary systems with asynchronous components. Without observed mutual events, their parameters cannot be estimated. Anyway, considering that we have observed three of them in two or three apparitions already - Metsahovi was followed up in 2009 and 2014, Kastel' in 2012 and 2014, and Gingasen in 2012 - but still did not detect mutual events — an unlikely situation if they were close binaries - , we consider that they are likely wide or semi-wide systems.

\subsection{Fully (doubly) synchronous binary asteroids}

While most known binary asteroids have asynchronously rotating primaries (and the size ratio $D_{2} / D_{1}$ substantially lower than 1 ), there exists a smaller group of binary systems where both components are in synchronous rotation. Their common feature is that they have high size (mass) ratios, $D_{2} / D_{1}>0.8$ for well described systems. With the secondary almost as large (massive) as the primary, a tidal synchronization timescale is short enough so that also the primary is locked in 1:1 spin-orbit resonance. An orbital dynamics of these doubly synchronous binaries is different from the dynamics of binary systems with asynchronous primaries, and it is outside the scope of this paper. Nevertheless, we summarize a few basic observations for them here.

We know 14 doubly synchronous binary asteroids. Two of them are large asteroids: (90) Antiope and (617) Patroclus with $D_{1} \sim D_{2}$ about 80 and $120 \mathrm{~km}$, respectively (Bartczak et al., 2014; Buie et al., 2015). Eleven are main belt asteroids with primary diameters between 4 and $13 \mathrm{~km}$. One, (69230) Hermes is a near-Earth asteroid with $D_{1}$ about $0.6 \mathrm{~km}$. Their periods $P_{\text {orb }}=P_{1}=P_{2}$ are from $13.89 \mathrm{~h}$ for (69230) Hermes to $118 \mathrm{~h}$ for (4951) Iwamoto (Pravec et al., 2006; Reddy et al., 2007). Primary and secondary shapes were derived for the two large systems (90) Antiope and (617) Patroclus for which also stellar occultation and adaptive optics observations were taken (see the two references above) and for (809) Lundia where a large amount of photometric data were obtained (Kryszczyńska et al., 2009, 2014). 


\section{Secondary rotation trends}

In this section, we describe several trends in the secondary properties that we reveal from our sample and compare them to theoretical predictions.

\section{$5.1 P_{s}$ vs $P_{\text {orb }}$ trends in $a / D_{1}$ and eccentricity}

Overall, synchronous rotations predominate in secondaries on close and nearcircular orbits, while asynchronous rotations are observed in secondaries on wider and/or eccentric orbits.

Synchronous secondaries of asteroid systems with asynchronous primaries are observed on close orbits with $a / D_{1} \lesssim 3.5, P_{\text {orb }}<43 \mathrm{~h}$. However, we note that the apparent lack of synchronous secondaries on wider orbits may be an observational bias at least partially, as slowly rotating synchronous secondaries on wider orbits could be more difficult to establish. The bias is because photometric observations of longer secondary periods are more demanding.

Asynchronous secondaries are observed at nearly all $a / D_{1}\left(P_{\text {orb }}\right)$ values, except the closest orbits with $a / D_{1} \lesssim 2.2\left(P_{\text {orb }} \lesssim 20 \mathrm{~h}\right)$. This lower limit appears significant, as asynchronous secondary rotations in closer orbits would be easy to detect if they existed. Further, we note that this lower limit is set by the observations of asynchronous secondaries in four small $\left(D_{1}=0.3-1.2 \mathrm{~km}\right)$ near-Earth asteroids and that it appears shifted to higher $a / D_{1}\left(P_{\text {orb }}\right)$ values for larger $\left(D_{1}=2-10 \mathrm{~km}\right)$ main-belt asteroid systems where the data suggest that asynchronous secondaries are present in semi-wide and wide orbits only. An exact lower limit on $a / D_{1}\left(P_{\text {orb }}\right)$ for asynchronous secondaries in main-belt asteroid systems will have to be established with future observations, but the current data suggest that it could be at $P_{\text {orb }}$ of a few days $\left(a / D_{1} \approx 5\right)$.

There is a significant anti-correlation of secondary synchroneity with eccentricity of its orbit around the primary. Synchronous secondaries are on nearcircular orbits; in all observed cases, the data are consistent with zero eccentricity (within 3- $\sigma$ uncertainty intervals). Asynchronous secondaries, on the other hand, are typically observed on eccentric orbits.

These observations are in agreement with the theories mentioned in Section 2 that predict that synchronous secondary rotations exist on low-eccentricity orbits while there exists a region of asynchronous or chaotic rotation in the phase space for moderate or large eccentricities. A reason for why asynchronous secondaries are observed on wider orbits may be longer tidal circularization time scales of secondaries at higher semi-major axes ${ }^{8}$, possibly combined with their

\footnotetext{
8 The tidal circularization of the secondary orbit is a slower process than tidal synchronization of secondary rotation, so the circularization time scale is relevant here.
} 
higher vulnerability to external perturbations on the wider orbits. From Fang and Margot (2012, Eq. 1), we derive the tidal circularization timescale:

$$
\tau_{\text {circ }}=\frac{512 \sqrt{2}\left(\frac{a}{D_{1}}\right)^{\frac{13}{2}} Q}{\left(84 k_{2}-57 k_{1} \frac{D_{2}}{D_{1}}\right) \omega_{\mathrm{d}} \sqrt{1+\left(\frac{D_{2}}{D_{1}}\right)^{3}}\left(\frac{D_{2}}{D_{1}}\right)^{2}},
$$

where $Q$ is the tidal quality factor, $k_{i}$ is the tidal Love number of the $i$ th component, $\omega_{\mathrm{d}} \equiv[(4 / 3) \pi \rho G]^{1 / 2}$ is the breakup spin rate ${ }^{9}$ and $\rho$ is the bulk density. ${ }^{10}$ Assuming $k_{i} \propto D_{i}$ for rubble pile (Goldreich and Sari, 2009), we obtain

$$
\tau_{\text {circ }}=\frac{512 \sqrt{2}\left(\frac{a}{D_{1}}\right)^{\frac{13}{2}} Q}{27 k_{2} \omega_{\mathrm{d}} \sqrt{1+\left(\frac{D_{2}}{D_{1}}\right)^{3}}\left(\frac{D_{2}}{D_{1}}\right)^{2}} .
$$

Taking a typical $D_{2} / D_{1}=1 / 3$ for binaries in our sample and assuming $Q=30$, $\omega_{\mathrm{d}}=7.5 \times 10^{-4} \mathrm{~s}$ (for $\rho=2 \mathrm{~g} \mathrm{~cm}^{-3}$ ), $k_{2}=10^{-5}$ and $10^{-6}$ for the typical sizes of main belt asteroid (MBA) and near-Earth asteroid (NEA) binaries per Goldreich and Sari (2009), respectively, we obtain a typical

$$
\tau_{\text {circ }} \approx\left[10^{12} \mathrm{~s}, 10^{13} \mathrm{~s}\right]\left(\frac{a}{D_{1}}\right)^{\frac{13}{2}} \doteq\left[3 \times 10^{4} \mathrm{yr}, 3 \times 10^{5} \mathrm{yr}\right]\left(\frac{a}{D_{1}}\right)^{\frac{13}{2}}
$$

respectively, for the MBA, NEA binaries in our sample. For the lower limits on relative semimajor axes for asynchronous secondaries of MBA and NEA binaries of $a / D_{1} \sim 5$ and 2.2, we obtain their typical tidal circularization timescales of $1 \times 10^{9} \mathrm{yr}$ and $5 \times 10^{7} \mathrm{yr}$, respectively. ${ }^{11}$ This suggests that the observation that asynchronous secondaries are on typically wider orbits in MBA binaries than in NEA binaries may be due to that the former are substantially older.

It is interesting that the (inner) satellites of the three main-belt asteroids (44620) 1999 RS43, (80218) 1999 VO123 and (8306) Shoko that are involved in asteroid pairs 44620-295745, 80218-213471 and 8306-2011 SR158 where the unbound secondaries (295745), (213471) and 2011 SR158 separated only about

\footnotetext{
9 The breakup spin rate is a rotation rate of a homogeneous sphere with zero tensile strength and the angle of repose of $90^{\circ}$ when the vectorial sum of the gravity force and the centrifugal force at the equator is zero.

${ }^{10} \mathrm{We}$ assume the same bulk density and quality factor for the primary and secondary, and we approximate the mass ratio $q=\left(D_{2} / D_{1}\right)^{3}$.

11 The tidal circularization timescales may be substantially shorter than the calculated values if real $Q / k_{2}$ is lower than the assumed values, see Goldreich and Sari (2009) for discussion on possible values of $Q$ and $k$ for rubble piles. Also, in the tidal circularization timescale derivation, it was assumed that the secondary is in synchronous rotation (Goldreich and Sari, 2009), so the derived timescales may not be correct for asynchronous secondaries.
} 
100-800 kyr ago appear to be in synchronous states. This suggests that either they were in their orbits much longer before the unbound secondaries separated, or their tidal circularization times are much shorter than estimated above. The latter might be because the approximation of the dynamical Love numbers with their static counterparts in Goldreich and Sari (2009) may not be right as suggested by Efroimsky (2015).

\subsection{Secondary rotational states}

We have found that of all the theoretically possible spin-orbit resonance states (with $p$ equal to an integer multiple of $+1 / 2$; see Section 2 ), only the 1:1 resonance $(p=+1)$ is populated. Other resonance states are not observed or they are unlikely.

The synchronous secondaries have their long axes aligned with the line connecting the centers of the primary and the secondary to within $20^{\circ}$, i.e., within the resolution limit of the photometric method for the librational angle (see Section 3), on most epochs. In two of the 24 synchronous secondaries in Section 4.1, there was observed a possible libration of $40^{\circ}-50^{\circ}$, but this will have to be confirmed with additional observations in the future.

The observed rotations of asynchronous secondaries are faster than their orbital rates. Their rotation periods are typically $<10 \mathrm{~h}$, and almost always $<20 \mathrm{~h}$, with one possible exception of the candidate outer satellite of 8306 Shoko that has $P_{3} \sim 61 \mathrm{~h}$. However, the tendency to rapid asynchronous secondary rotations may be an observational bias, as slow secondary rotations are difficult to observationally establish, see comments in the second paragraph of Section 5.1 above.

It is notable that all, with one possible exception, the observed secondary rotations appear single-periodic with no obvious signs of a chaotic rotation, and that their periods appear constant both within a single apparition (lasting typically weeks to months) as well as over different apparitions (years). When re-observed, the best fit secondary periods, both synchronous and asynchronous, are the same with a relative accuracy of $10^{-2}$ or better. The exception is (35107) $1991 \mathrm{VH}$ where the secondary period observed in February 2003 did not fit for the data obtained in 2008, suggesting a non-constant secondary rotation.

In comparing these observations with the theories mentioned in Section 2, it stands out that we observed only 1:1 synchronous states, but not the other theoretically possible resonance states. It is probably because locking into any other than 1:1 resonance requires an eccentric orbit ${ }^{12}$ and none of the synchronous secondaries in our sample have orbits with observable eccentricity. The paucity of chaotic secondary rotations is particularly intriguing. In the future we will investigate whether there might be an observational bias against

\footnotetext{
12 The other than 1:1 spin-orbit resonance states require a non-zero eccentricity as $H(p, 0)=1$ for $p=+1$ and 0 for other $p$ (Murray and Dermott, 1999).
} 
their detection. For that, it could be useful to study theoretically what is the frequency spectrum of a chaotically rotating satellite.

\subsection{Coupled spin-orbit simulations}

One of us (SPN) used the integrator developed in Naidu and Margot (2015) to simulate the coupled spin-orbit dynamics in binary systems listed in Table 1 that have synchronous secondaries. In each system, we assumed the two bodies to be ellipsoids with dimensions that satisfied the parameters provided in Table 1. For the purpose of these simulations, we chose the equatorial axes $a_{i}$ and $b_{i}$ such that $\left(a_{i}+b_{i}\right) / 2=D_{i}$ and $a_{i} / b_{i}$ equals the observed values given in Table 1. The polar axis, $c_{i}$, was chosen to be equal to $b_{i}$. $(i=1$ and 2 for the primary and the secondary, respectively.) Although attitude stability requires that $c$ be smaller than the equatorial axes, our choice of $c$ does not affect the attitude stability in these simulations because all the systems are assumed to be planar, i.e., both bodies are in principal axis rotation about their $c$ axes and their equatorial planes are aligned with the mutual orbit at all times. System masses were computed from the orbital periods and semimajor axes using Kepler's Law. ${ }^{13}$ The primary and secondary were assumed to have equal densities, which were computed by dividing the system mass by the system volume (primary + secondary). We started all simulations at the pericenter of the osculating mutual orbit and with the longest axis of each body pointing toward each other. The semimajor axis value and the upper limit value for eccentricity given in Table 1 were used for the initial osculating orbit. We simulated each binary system with various initial satellite spin rates (close to synchronous) to find the relaxed state of the satellite (Naidu and Margot, 2015). The relaxed state libration amplitude corresponds to the forced + optical libration amplitude in the case of a decoupled treatment of the satellite spin (e.g., Murray and Dermott, 1999). The libration estimates are given in Table 4. Note that since the eccentricities given in Table 1 are $3-\sigma$ upper limits, the libration amplitude estimates are also upper limits. In the case of (7088) Ishtar, (17260) 2000 JQ58, and (80218) 1999 VO123, the libration amplitudes reached $90^{\circ}$ and the satellites did not stay synchronous in the simulations. This suggests that their orbital eccentricities are actually smaller than the upper limit estimates. We examined how uncertainties in the binary parameters propagate to the libration amplitude estimates and found that the biggest source of uncertainty is the secondary elongation $a_{2} / b_{2}$. For instance, for Sevastopol and Mayall, the libration amplitude uncertainties due to $a_{2} / b_{2}$ uncertainty are around $10^{\circ}$ whereas uncertainties due to other factors are on an order of $2^{\circ}$. The observed phase offsets given in Table 2 are mostly consistent with the estimates from these simulations. In the case of (5481) Kiuchi, (5905) Johnson, and (1453) Fennia, the observed maximum phase offsets are greater by $5^{\circ}-7^{\circ}$ than the simulation estimates. This may due to presence of irregular shape effects that can cause systematic shifts of the secondary rotational minima by up to $10^{\circ}-20^{\circ}$ (see Section 3 ) or due to the uncertainties

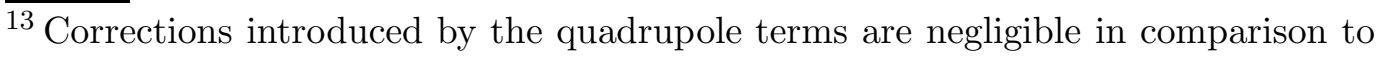
uncertainties in other parameters.
} 
in their $a_{2} / b_{2}$ estimates. We conclude that the observations and the coupled spin-orbits dynamics theory are fully consistent for the observed systems with synchronous secondaries.

\subsection{Secondary elongations}

Synchronous secondaries have shapes with low to moderate equatorial elongations. Figure 43 shows the estimated equatorial axis ratios for 22 synchronous secondaries from Table 1 . There is an observational bias against low elongations with $a_{2} / b_{2} \lesssim 1.2$ due to the difficulty to detect very small secondary rotational amplitudes in binary asteroid lightcurves; the apparent paucity of secondaries with near-spheroidal shapes in the sample may be due to the observational selection effect. The lack of synchronous secondaries with elongations $a_{2} / b_{2} \gtrsim 1.5$ is significant; if there were secondaries with greater elongations, we would detect them easily.

Data on elongations of asynchronous secondaries are scarce. They were constrained for only four cases in Section 4.2. Three of them have the equatorial axis ratios about 1.3, i.e., similar to the elongations of synchronous secondaries. The asynchronous outer secondary of (2577) Litva may have a greater elongation, but the estimate has a high error and it needs to be confirmed with more accurate observations in the future.

The lack of synchronous secondaries with estimated equatorial elongations $a_{2} / b_{2} \gtrsim 1.5$ seems to be consistent with the theoretical predictions mentioned in Section 2 of that there exist large regions of chaotic rotation in the phase space for $(\mathcal{B}-\mathcal{A}) / \mathcal{C} \gtrsim 1 / 3$, which corresponds to the axial ratio of dynamically equivalent ellipsoid $a_{2} / b_{2} \gtrsim \sqrt{2}$. From Fig. 43 , it is apparent that all the estimated axial ratios are consistent with this theoretical upper limit, considering their error bars. Alternatively, the moderately elongated secondary shapes may be that they do not form or stay very elongated in gravitational (tidal) field from the primary. This would explain also the apparent paucity (if it is confirmed on a larger sample in the future) of highly elongated shapes among asynchronous secondaries. Jacobson and Scheeres (2011a) suggested that asteroid satellites can undergo a secondary fission during chaotic evolution of asteroid system after rotational fission of critically spinning parent asteroid. They found that a fraction of secondaries that undergo secondary fission is positively correlated with the secondary elongation $a_{2} / b_{2}$. In particular, their simulations of the secondary fission process gave that the fraction of fissioned secondaries was $<0.4$ for $a_{2} / b_{2}<1.4$, but it increased to $>0.5$ for $a_{2} / b_{2} \gtrsim 1.5$ (Jacobson and Scheeres, 2011a, section 3.5 and Fig. 8). Sharma (2009, 2014) investigated equilibria of rubble pile satellites and their stability. He assumed that the bodies are strenghtless aggregates and he modeled their plastic deformations, considering two parameters: the angle of friction and the plastic modulus. He found that prolate and triaxial satellites with the internal friction angle of $30^{\circ}$ and equatorial elongations $a_{2} / b_{2} \lesssim 1.5$ are more stable to finite structural perturbations than more elongated ones. These works indicate that more elongated secondaries, if they are originally formed in spin fission of par- 
ent asteroid, are less likely to survive intact and they do more frequently fail or fission.

\subsection{Primary rotations and elongations}

Asynchronously rotating primaries have specific spin and shape characteristics, largely independent of whether their satellites are synchronous or asynchronous. Specifically, all the asteroid systems with synchronous secondaries have fast rotating primaries with periods between 2.2 and $4.5 \mathrm{~h}$ and with nearly spheroidal shapes with $a_{1} / b_{1}=1.0-1.1$ mostly, and always $\leq 1.21$. These synchronous systems have all the total angular momentum close to the critical limit for a single body in a gravity regime with the normalized total angular momentum $\alpha_{L}$ around 1.0 (see Pravec and Harris, 2007). ${ }^{14}$ Primaries with asynchronous satellites show similar characteristics. They have nearly spheroidal shapes with $a_{1} / b_{1} \leq 1.2$. Most of them have rotational periods $P_{1}<4.5 \mathrm{~h}$ and the near-critical total angular momentum as expected for asteroid systems formed by spin-up fission of cohesionless parent bodies, but there are exceptions among systems with suspected asynchronous satellites on (semi-)wide orbits. Of the 10 systems presented in Sections 4.2 .3 and 4.2.4, four have slower rotating primaries with $P_{1}=4.8-6.6 \mathrm{~h}$. These systems may be more evolved than systems with satellites on closer orbits; they appear to have more angular momentum transferred from the primary's rotation to the secondary's orbit. At least one of them, (32039) 2000 JO23 seems to have a super-critical total angular momentum; we estimate its $\alpha_{L}>2$. That is, the system contains twice (or more) as much angular momentum than could be contained in the original parent asteroid at the time of its rotational fission. This suggests that an additional angular momentum was provided by some mechanism that moved the satellite to wider orbit since formation.

\section{Concluding remarks}

Some of the significant trends in secondary rotations and elongations that we revealed from our observations appear consistent with the theories as discussed above. However, we found also certain inconsistencies. The most significant one seems to be the apparent mismatch between the observations of synchronous secondaries suggesting short tidal evolution timescales and the prediction from the classical tidal theory by Goldreich and Sari (2009) that gives much longer timescales. It calls for a re-thinking of the tidal energy dissipation in asteroid binary systems. While the present theories of tidal evolution (see also Taylor and Margot, 2011, and references therein) assume an elastic response of the asteroid material to the tidal forces, the data suggesting much

\footnotetext{
$\overline{14}$ The normalized total angular momentum $\alpha_{L}=1$ is for a critically spinning equivalent sphere (i.e., a sphere of the same total mass and volume as the two components of the binary system) with the angle of friction of $90^{\circ}$ (Pravec and Harris, 2007).
} 
shorter timescales may indicate a non-elastic behavior due to the large amplitude of the tidal forcing function. Alternatively, a low-load friction theory (a "tribology theory") may be needed to be developed for rubble pile asteroids as suggested by Jacobson and Scheeres (2011b). Another interesting result is the paucity of chaotic secondary rotations in our sample. While we will investigate possible observational biases against their detection, work on improving the theories of spin-orbit coupling is called for. In particular, inclusion of tidal torque dynamics and modeling of rheology of the bodies may be needed.

\section{Acknowledgements}

We thank to Alan Harris, Seth Jacobson, Michael Efroimsky, Matija Ćuk, Ishan Sharma and Masatoshi Hirabayashi for fruitful discussions on a number of things in this paper. The work at Ondřejov and observations with the Danish 1.54-m telescope on the ESO La Silla station were supported by the Grant Agency of the Czech Republic, Grants P209/12/0229 and 15-07193S. The work at Modra was supported by the Slovak Grant Agency for Science VEGA, Grant 1/0670/13. Operations at Sugarloaf Mountain were supported by a Gene Shoemaker NEO grant from the Planetary Society. The work at Tatranská Lomnica was supported by the Slovak Grant Agency for Science VEGA, Grant 2/0032/14 and project ITMS No. 26220120009, based on the supporting operational Research and development program financed from the European Regional Development Fund. The work at SETI Institute was supported by NASA grants NNX11AD62G and NNX14AJ80G. The work at Abastumani was supported by the Shota Rustaveli National Science Foundation, Grant FR/379/6-300/14. We thank to S. Nudds for her contribution with observations from Elginfield Observatory, University of Western Ontario.

\section{References}

Bartczak, P., Michalowski, T., Santana-Ros, T., Dudziński, G., 2014. A new non-convex model of the binary asteroid 90 Antiope obtained with the SAGE modelling technique. Mon. Not. Royal Astron. Soc. 443, 1802-1809.

Becker, T. M., Howell, E. S., Nolan, M. C., Magri, C., Pravec, P., Taylor, P. A., Oey, J., Higgins, D., Világi, J., Kornoš, L., Galád, A., Gajdoš, Š., Gaftonyuk, N. M., Krugly, Y. N., Molotov, I. E., Hicks, M. D., Carbognani, A., Warner, B. D., Vachier, F., Marchis, F., Pollock, J. T., 2015. Physical modeling of triple near-Earth Asteroid (153591) 2001 SN263 from radar and optical light curve observations. Icarus 248, 499-515.

Benner, L. A. M., Nolan, M. C., Margot, J.-L., Ostro, S. J., Giorgini, J. D., 2003. Radar Imaging of Binary Near-Earth Asteroid 1998 ST27. Bull. Amer. Astron. Soc. 35, 959.

Brooks, H. E., 2006. Orbits of Binary Near-Earth Asteroids from Radar Observations. Bull. Amer. Astron. Soc. 38, 934.

Brozović, M., Benner, L. A. M., Taylor, P. A., Nolan, M. C., Howell, E. S., 
Magri, C., Scheeres, D. J., Giorgini, J. D., Pollock, J. T., Pravec, P., Galád, A., Fang, J., Margot, J.-L., Busch, M. W., Shepard, M. K., Reichart, D. E., Ivarsen, K. M., Haislip, J. B., Lacluyze, A. P., Jao, J., Slade, M. A., Lawrence, K. J., Hicks, M. D., 2011. Radar and optical observations and physical modeling of triple near-Earth Asteroid (136617) 1994 CC. Icarus 216, 241-256.

Buie, M. W., et al., 2015. Size and shape from stellar occultation observations of the double Jupiter Trojan Patroclus and Menoetius. Astron. J. 149, article id. 113 (11 pp).

Cooney, W., Gross, J., Terrell, D., Stephens, R., Pravec, P., Kušnirák, P., Durkee, R., Galád, A., 2006a. (1717) Arlon. CBET 369.

Cooney, W., Gross, J., Terrell, D., Pravec, P., Kušnirák, P., Pray, D., Krugly, Yu., Kornoš, L., Világi, J., Gajdoš, S., Galád, A., Reddy, V., Dyvig, R., Nudds, S., Kanuchova, Z., Pikler, M., Husárik, M., 2006b. (1717) Arlon. CBET 504.

Ćuk, M., Nesvorný, D., 2010. Orbital evolution of small binary asteroids. Icarus 207, 732-743.

Efroimsky, M., 2015. Tidal evolution of asteroidal binaries. Ruled by viscosity. Ignorant of rigidity. Astron. J. 150, 98 (12 pp).

Goldreich, P., Sari, R., 2009. Tidal evolution of rubble piles. Astrophys. J. 691, 54-60.

Fang, J., Margot, J.-L., Brozovic, M., Nolan, M. C., Benner, L. A. M., Taylor, P. A., 2011. Orbits of Near-Earth Asteroid Triples 2001 SN263 and 1994 CC: Properties, Origin, and Evolution. Astron. J. 141, article id. 154, 15 pp.

Fang, J., Margot, J.-L., 2012. Near-Earth binaries and triples: Origin and evolution of spin-orbital properties. Astron. J. 143, article id. 24, 14 pp.

Harris, A. W., Young, J. W., Scaltriti, F., Zappala, V., 1984. Lightcurves and phase relations of the asteroids 82 Alkmene and 444 Gyptis. Icarus 57, 251-258.

Harris, A. W. et al., 2014. On the maximum amplitude of harmonics of an asteroid lightcurve. Icarus 235, 55-59.

Higgins, D., P. Pravec, P. Kušnirák, A. Galád, S. Gajdoš, J. Világi, L. Kornoš, 2006. (17260) 2000 JQ58. CBET 431.

Higgins, D., Pravec, P., Kušnirák, P., Hornoch, K., Pray, D. P., Világi, J., Kornoš, L., Gajdoš, S., Husárik, M., Pikler, M., Červák, G., 2008. Asteroid Lightcurve Analysis of Suspected Binary Asteroids. Minor Planet Bull. 35, 173-175.

Higgins, D., Pravec, P., Kušnirák, P., Pollock, J., Oey, J., Husárik, M., Červák, G., Reichart, D. E., Ivarsen, K. M., Haislip, J. B., Lacluyze, A., 2010. (2121) Sevastopol. CBET 2427. 
Jacobson, S. A., Scheeres, D. J., 2011a. Dynamics of rotationally fissioned asteroids: Source of observed small asteroid systems. Icarus 214, 161-178.

Jacobson, S. A., Scheeres, D. J., 2011b. A long-term stable equilibrium for synchronous binaries including tides and the BYORP effect. AAS/Division on Dynamical Astronomy Meeting Abstracts 42, Talk: 01.02.

Jakubík, M., Husárik, M., Világi, J., Gajdoš, S., Galád, A., Pravec, P., Kušnirák, P., Cooney, W., Gross, J., Terrell, D., Pray, D., Stephens, R., 2005. (9260) Edwardolson. CBET 270.

Kryszczyńska, A., et al., 2009. New binary asteroid 809 Lundia. I. Photometry and modelling. Astron. Astrophys. 501, 769-776.

Kryszczyńska, A., et al., 2014. Non-convex model of the binary asteroid (809) Lundia and its density estimation. Asteroids, Comets, Meteors 2014. Proc. of the Asteroids, Comets, Meteors 2014 conference, Helsinki, Finland (Eds. K. Muinonen et al.)

Kušnirák, P., Pravec, P., Oey, J., Stephens, R., Husárik, M., Ries, J., 2008. (5481) Kiuchi. CBET 1339.

Margot, J. L., Nolan, M. C., Benner, L. A. M., Ostro, S. J., Jurgens, R. F., Slade, M. A., Giorgini, J. D., Campbell, D. B., 2000. Satellites of Minor Planets. IAU Circ. 7503.

Margot, J. L., Nolan, M. C., Benner, L. A. M., Ostro, S. J., Jurgens, R. F., Giogini, J. D., Slade, M. A., Campbell, D. B., 2002. Binary Asteroids in nearEarth object population. Science 296, 1445-1448.

Margot, J.-L., Pravec, P., Taylor, P., Carry, B., Jacobson, S., 2015. Asteroid Systems: Binaries, Triples, and Pairs. in ASTEROIDS IV. Eds. R. P. Binzel, P. Michel, F. E. DeMeo, W. F. Bottke, Jr., University of Arizona Press. (in press).

Merline, W. J., Close, L. M., Tamblyn, P. M., Menard, F., Chapman, C. R., Dumas, C., Duvert, G., Owen, W. M., Slater, D. C., Sterzik, M. F., 2003. S/2003 (1509) 1. IAU Circ. 8075.

Merline, W. J., Close, L. M., Dumas, C., Chapman, C. R., Menard, F., Tamblyn, P. M., Durda, D. D., 2003. Discovery of new asteroid binaries (121) Hermione and (1509) Esclangona. Bull. Amer. Astron. Soc. 35, 972.

Merline, W. J., Tamblyn, P. M., Warner, B. D., Pravec, P., Tamblyn, J. P., Neyman, C., Conrad, A. R., Owen, W. M., Carry, B., Drummond, J. D., Chapman, C. R., Enke, B. L., Grundy, W. M., Veillet, C., Porter, S. B., Arcidiacono, C., Christou, J. C., Durda, D. D., Harris, A. W., Weaver, H. A., Dumas, C., Terrell, D., Maley, P., 2013. S/2012 (2577) 1. IAU Circ. 9267.

Muinonen, K. and Lagerros, J. S. V., 1998. Inversion of shape statistics for small solar system bodies. Astronomy and Astrophysics 333, 753-761. 
Murray, C. D., and Dermott, S. F., 1999. Solar System Dynamics. (Cambridge: Cambridge Univ. Press)

Naidu, S. P., Margot, J. L., Busch, M. W., Taylor, P. A., Nolan, M. C., Howell, E. S., Giorgini, J. D., Benner, L. A. M., Brozovic, M., Magri, C., 2012. Dynamics of Binary Near-Earth Asteroid System (35107) 1991 VH. AAS/DDA meeting $43,7.07$

Naidu, S. P., Margot, J.-L., 2015. Near-Earth Asteroid Satellite Spins Under Spin-orbit Coupling. Astron. J. 149, article id. 80, 11 pp.

Naidu, S. P., Margot, J. L., Taylor, P. A., Nolan, M. C., Busch, M. W., Benner, L. A. M., Brozovic, M., Giorgini, J. D., Jao, J. S., Magri, C., 2015. Radar Imaging and Characterization of the Binary Near-Earth Asteroid (185851) 2000 DP107. Astron. J. 150, article id. 54, 12 pp.

Nolan, M. C., Howell, E. S., Miranda, G., 2004. Radar Images of Binary Asteroid 2003 YT1. Bull. Amer. Astron. Soc. 36, 1132.

Ostro, S. J., Margot, J.-L., Nolan, M. C., Benner, L. A. M., Jurgens, R. F., Giorgini, J. D., 2000. 2000 DP107. IAU Circ. 7496.

Ostro, S. J., Nolan, M. C., Benner, L. A. M., Giorgini, J. D., Margot, J. L., Magri, C., 2003. 1990 OS. IAU Circ. 8237.

Ostro, S.J., Margot, J.-L., Benner, L. A. M., Giorgini, J. D., Scheeres, D. J., Fahnestock, E. G., Broschart, S. B., Bellerose, J., Nolan, M. C., Magri, C., Pravec, P., Scheirich, P., Rose, R., Jurgens, R. F., De Jong, E. M., Suzuki, S., 2006. Radar imaging of binary near-Earth asteroid (66391) 1999 KW4. Science 314, 1276-1280.

Pikler, M., Husárik, M., Červák, G., Cooney, W., Gross, J., Terrell, D., Pravec, P., Kušnirák, P., Galád, A., Világi, J., Kornoš, L., Gajdoš, S., Reddy, V., Dyvig, R., 2007. (2486) Metsahovi. CBET 860.

Pravec, P. and Harris, A. W., 2007. Binary asteroid population. 1. Angular momentum content. Icarus 190, 250-259.

Pravec, P., Šarounová, L., Wolf, M., Mottola, S., Lahulla, F., 1998a. 1996 FG3. IAU Circ. 7074.

Pravec, P., Wolf, M., Šarounová, L., 1998b. Occultation/Eclipse Events in Binary Asteroid 1991 VH. Icarus 133, 79-88.

Pravec, P., Šarounová, L., Rabinowitz, D. L., Hicks, M. D., Wolf, M., Krugly, Y. N., Velichko, F. P., Shevchenko, V. G., Chiorny, V. G., Gaftonyuk, N. M., Genevier, G., 2000. Two-period lightcurves of 1996 FG $_{3}, 1998$ PG, and (5407) 1992 AX: One probable and two possible binary asteroids. Icarus 146, 190-203.

Pravec, P., Kušnirák, P., Šarounová, L., Brown, P., Esquerdo, G., Pray, D., Benner, L. A. M., Nolan, M. C., Giorgini, J. D., Ostro, S. J., Margot, J.-L., 
Pravec, P., Kušnirák, P., Šarounová, L., Brown, P., Kaiser, N., Masi, G., Mallia, F., 2004. 1999 DJ $_{4}$. IAU Circ. 8316.

Pravec, P., Harris, A. W., Scheirich, P., Kušnirák, P., Šarounová, L., Hergenrother, C. W., Mottola, S., Hicks, M. D., Masi, G., Krugly, Y. N., Shevchenko, V. G., Nolan, M. C., Howell, E. S., Kaasalainen, M., Galád, A., Brown, P., DeGraff, D. R., Lambert, J. V., Cooney Jr., W. R., Foglia, S., 2005a. Tumbling asteroids. Icarus 173 108-131.

Pravec, P., Kušnirák, P., et al., 2005b. (3982) Kastel. IAU Circ. 8609.

Pravec, P., Scheirich, P., Kušnirák, P., Šarounová, L., Mottola, S., Hahn, G., Brown, P., Esquerdo, G., Kaiser, N., Krzeminski Z., Pray, D. P., Warner, B. D., Harris, A. W., Nolan, M. C., Howell, E. S., Benner, L. A. M., Margot, J.-L., Galád, A., Holliday, W., Hicks, M. D., Krugly, Y. N., Tholen, D., Whiteley, R., Marchis, F., DeGraff, D. R., Grauer, A., Larson, S., Velichko, F. P., Cooney Jr., W. R., Stephens, R., Zhu, J., Kirsch, K., Dyvig, R., Snyder, L., Reddy, V., Moore, S., Gajdoš, Š., Világi, J., Masi, G., Higgins, D., Funkhouser, G., Knight, B., Slivan, S., Behrend, R., Grenon, M., Burki, G., Roy, R., Demeautis, C., Matter, D., Waelchli, N., Revaz, Y., Klotz, A., Rieugné, M., Thierry, P., Cotrez, V., Brunetto, L., Kober, G., 2006. Photometric survey of binary nearEarth asteroids. Icarus 181, 63-93.

Pravec, P., Vokrouhlický, D., Polishook, D., Scheeres, D. J., Harris, A. W., Galád, A., Vaduvescu, O., Pozo, F., Barr, A., Longa, P., Vachier, F., Colas, F., Pray, D. P., Pollock, J., Reichart, D., Ivarsen, K., Haislip, J., Lacluyze, A., Kušnirák, P., Henych, T., Marchis, F., Macomber, B., Jacobson, S. A., Krugly, Y. N., Sergeev, A. V., Leroy, A., 2010. Formation of asteroid pairs by rotational fission. Nature 466, 1085-1088.

Pravec, P., Scheirich, P., Vokrouhlický, D., Harris, A. W., Kušnirák, P., Hornoch, K., Pray, D. P., Higgins, D., Galád, A., Világi, J., Gajdoš, Š., Kornoš, L., Oey, J., Husárik, M., Cooney, W. R., Gross, J., Terrell, D., Durkee, R., Pollock, J., Reichart, D. E., Ivarsen, K., Haislip, J., Lacluyze, A., Krugly, Y. N., Gaftonyuk, N., Stephens, R. D., Dyvig, R., Reddy, V., Chiorny, V., Vaduvescu, O., Longa-Pena, P., Tudorica, A., Warner, B. D., Masi, G., Brinsfield, J., Gonçalves, R., Brown, P., Krzeminski, Z., Gerashchenko, O., Shevchenko, V., Molotov, I., Marchis, F., 2012. Binary asteroid population. 2. Anisotropic distribution of orbit poles of small, inner main-belt binaries. Icarus 218, 125143.

Pravec, P., Kušnirák, P., Hornoch, K., Galád, A., Krugly, Yu. N., Chiorny, V., Inasaridze, R., Kvaratskhelia, O., Ayvazian, V., Parmonov, O., Pollock, J., Mottola, S., Oey, J., Pray, D., Žižka, J., Vraštil, J., Molotov, I., Reichart, D. E., Ivarsen, K. M., Haislip, J. B., LaCluyze, A., 2013. (8306) Shoko. IAU Circ. 9268.

Pray, D., Pravec, P., Kušnirák, P., Gajdoš, S., Világi, J., Galád, A., Nudds, S., Krzeminski, Z., 2005. (114319) 2002 XD58. CBET 328. 
Pray, D., Pravec, P., Kušnirák, P., Hornoch, K., Krugly, Yu., Chiorny, V., Husárik, M., Pikler, M., Világi, J., Gajdoš, S., Galád, A., Cooney, W., Gross, J., Terrell, D., Gaftonyuk, N., Stephens, R., Durkee, R., Marchis, F., Baek, M., Macomber, B., Reddy, V., Colas, F., Lecacheux, J., Ries, J., 2007a. (16635) 1993 QO. CBET 1143.

Pray, D., Pravec, P., Kušnirák, P., Hornoch, K., Higgins, D., Cooney, W., Gross, J., Terrell, D., Oey, J., Galád, A., Kornoš, L., Gajdoš, S., Világi, J., Stephens, R., Marchis, F., Baek, M., Macomber, B., Reddy, V., Husárik, M., Pikler, M., 2007b. (32039) 2000 JO23. CBET 1147.

Pray, D., Pravec, P., Chiorny, V., Krugly, Yu. N., Benishek, V., Pollock, J., Caton, D. B., Smith, A. B., Hawkins, R. L., Inasaridze, R., Montaigut, R., Leroy, A., Reichart, D. E., Haislip, J. B., LaCluyze, A. P., 2014. (2623) Zech. CBET 4012.

Reddy, V., Dyvig, R., Pravec, P., Kušnirák, P., Gajdoš, S., Galád, A., Kornoš, L., 2006. (7088) Ishtar. CBET 384.

Reddy, V., et al., 2007. (4951) Iwamoto. IAU Circ. 8836.

Scheirich, P. and Pravec, P., 2009. Modeling of lightcurves of binary asteroids. Icarus 200, 531-547.

Scheirich, P., Pravec, P., Jacobson, S. A., Ďurech, J., Kušnirák, P., Hornoch, K., Mottola, S., Mommert, M., Hellmich, S., Pray, D., Polishook, D., Krugly, Yu. N., Inasaridze, R. Ya., Kvaratskhelia, O. I., Ayvazian, V., Slyusarev, I., Pittichová, J., Jehin, E., Manfroid, J., Gillon, M., Galád, A., Pollock, J., Licandro, J., Alí-Lagoa, V., Brinsfield, J., Molotov, I. E., 2015a. The binary near-Earth Asteroid (175706) 1996 FG3 - An observational constraint on its orbital evolution. Icarus 245, 56-63.

Scheirich, P., et al. Binary asteroid population. 4. Predominance of retrograde orbital poles of binary asteroids. In preparation.

Sharma, I., 2009 The equilibrium of rubble-pile satellites: The Darwin and Roche ellipsoids for gravitationally held granular aggregates. Icarus 200, 636654.

Sharma, I., 2014. Stability of rubble-pile satellites. Icarus 229, 278-294.

Shepard, M. K., Margot, J.-L., Magri, C., Nolan, M. C., Schlieder, J., Estes, B., Bus, S. J., Volquardsen, E. L., Rivkin, A. S., Benner, L. A. M., Giorgini, J. D., Ostro, S. J., Busch, M. W., 2006, Radar and infrared observations of binary near-Earth Asteroid 2002 CE26. Icarus 184, 198-210.

Shepard, M. K., Nolan, M. C., Benner, L. A. M., Giorgini, J. D., Ostro, S. J., Magri, C., 2008. 2005 NB . IAU Circ. 8936.

Taylor, P. A., Margot, J. L., Nolan, M. C., Benner, L. A. M., Ostro, S. J., Giorgini, J. D., Magri, C., 2008. The Shape, Mutual Orbit, and Tidal Evo- 
lution of Binary Near-Earth Asteroid 2004 DC. LPI Contribution No. 1405, paper id. 8322.

Taylor, P. A., Margot, J.-L., 2011. Binary asteroid systems: Tidal end states and estimates of material properties. Icarus 212, 661-676.

Vokrouhlický, D. and Nesvorný, D., 2008. Pairs of Asteroids Probably of a Common Origin. Astron. J. 136, 280-290.

Warner, B. D., Pravec, P., Kušnirák, P., Cooney, W., Gross, J., Terrell, D., Higgins, D., Kornoš, L., Világi, J., Pray, D., Masi, G., Mallia, F., 2005a. (5477) $1989 \mathrm{UH}_{2}$. CBET 288.

Warner, B. D., Pravec, P., Kušnirák, P., Pray, D., Galád, A., Gajdoš, S., Brown, P., Krzeminski, Z., 2005b. (5905) Johnson. IAU Circ. 8511.

Warner, B. D., Pravec, P., Pray, D., 2005c. (76818) 2000 RG 79 . IAU Circ. 8592.

Warner, B. D., Pravec, P., Kušnirák, P., Cooney, W., Gross, J., Terrell, D., Nudds, S., 2005d. (3309) Brorfelde. CBET 279.

Warner, B. D., Harris, A. W., Pravec, P., Stephens, R. D., Pray, D., Cooney, W., Gross, J., Terrell, D., Gajdoš, S., Galád, A., Krugly, Yu., 2007, (1453) Fennia. CBET 1150.

Warner, B., Pravec, P., Kušnirák, P., Hornoch, K., Világi, J., Kornoš, L., Gajdoš, S., Veres, P., Galád, A., Cooney, W., Gross, J., Terrell, D., Krugly, Yu., Chiorny, V., 2009. (2131) Mayall. CBET 2105.

Warner, B. D., Pravec, P., Kušnirák, P., Hornoch, K., Harris, A., Stephens, R. D., Casulli, S., Cooney, W. R., Jr., Gross, J., Terrell, D., Durkee, R., Gajdoš, Š., Galád, A., Kornoš, L., Tóth, J., Világi, J., Husárik, M., Marchis, F., Reiss, A. E., Polishook, D., Roy, R., Behrend, R., Pollock, J., Reichart, D., Ivarsen, K., Haislip, J., Lacluyze, A., Nysewander, M., Pray, D. P., Vachier, F., 2010. A Trio of Hungaria Binary Asteroids. Minor Planet Bull. 37, 70-73.

Warner, B. D., 2014. Binary Asteroid Lightcurve Analysis at the CS3-Palmer Divide Station: 2013 June-September. Minor Planet Bull. 41, 54-57. 
Table 1

Binary asteroids with synchronous secondaries

\begin{tabular}{|c|c|c|c|c|c|c|c|c|c|c|c|c|}
\hline Binary system & $\begin{array}{l}D_{1} \\
(\mathrm{~km}) \\
\end{array}$ & $\frac{D_{2}}{D_{1}}$ & $\frac{a}{D_{1}}$ & $e$ & $\begin{array}{l}P_{1} \\
\text { (h) } \\
\end{array}$ & $\begin{array}{c}P_{\text {orb }} \\
(\mathrm{h})\end{array}$ & $\begin{array}{l}P_{2} \\
\text { (h) }\end{array}$ & $\begin{array}{c}A_{1} \\
(\mathrm{mag})\end{array}$ & $\begin{array}{c}A_{2} \\
(\mathrm{mag})\end{array}$ & $\begin{array}{r}\text { SolPh } \\
\left({ }^{\circ}\right)\end{array}$ & $\frac{a_{1}}{b_{1}}$ & $\frac{a_{2}}{b_{2}}$ \\
\hline (2121) Sevastopol & 8.6 & 0.41 & 3.3 & $<0.03$ & 2.90660 & 37.1535 & 37.13 & 0.16 & 0.069 & 13 & 1.15 & 1.42 \\
\hline (2131) Mayall & 8.2 & 0.30 & 2.4 & $<0.20$ & 2.5678 & 23.4849 & 23.47 & 0.09 & 0.035 & 23 & 1.06 & 1.31 \\
\hline (5477) Holmes & 3.0 & 0.39 & 2.5 & $<0.05$ & 2.9940 & 24.40369 & 24.40 & 0.10 & 0.051 & 12 & 1.09 & 1.33 \\
\hline (5481) Kiuchi & 3.6 & 0.35 & 2.2 & $<0.18$ & 3.6196 & 20.90621 & 20.91 & 0.10 & 0.036 & 9 & 1.09 & 1.30 \\
\hline (5905) Johnson & 4.48 & 0.38 & 2.3 & $<0.06$ & 3.7823 & 21.79701 & 21.76 & 0.16 & 0.035 & 22 & 1.12 & 1.19 \\
\hline (7088) Ishtar & 1.05 & 0.42 & 2.2 & $<0.16$ & 2.6786 & 20.63 & 20.60 & 0.11 & 0.09 & 20 & 1.09 & 1.49 \\
\hline (44620) 1999 RS43 & 2.0 & 0.34 & 3.1 & & 3.1401 & 33.63 & 33.2 & 0.10 & 0.037 & 8 & 1.09 & 1.33 \\
\hline (66063) 1998 RO1 & 0.8 & 0.48 & 1.8 & $<0.06$ & 2.4924 & 14.54578 & 14.52 & 0.14 & 0.119 & 25 & 1.11 & 1.48 \\
\hline (66391) 1999 KW4 & 1.28 & 0.33 & 1.99 & $<0.006$ & 2.7645 & 17.42 & 17.4 & & & & 1.04 & 1.32 \\
\hline (76818) 2000 RG79 & 2.5 & 0.34 & 1.7 & $<0.13$ & 3.1665 & 14.12994 & 14.132 & 0.13 & 0.052 & 16 & 1.11 & 1.42 \\
\hline (85938) 1999 DJ4 & 0.35 & 0.5 & 2.1 & & 2.5141 & 17.73 & 17.70 & 0.10 & 0.13 & 63 & 1.05 & 1.31 \\
\hline (175706) 1996 FG3 & 1.64 & 0.29 & 1.5 & $<0.07$ & 3.595195 & 16.1508 & 16.15 & 0.08 & 0.030 & 17 & 1.06 & 1.31 \\
\hline (185851) 2000 DP107 & 0.86 & 0.40 & 3.1 & $<0.05$ & 2.7745 & 42.13 & 42.5 & & & & 1.03 & 1.20 \\
\hline (399774) 2005 NB7 & 0.5 & 0.34 & 1.8 & & 3.488 & 15.28 & 15.28 & 0.11 & 0.09 & 57 & 1.05 & 1.46 \\
\hline \multicolumn{13}{|c|}{ Probable synchronous secondaries: } \\
\hline (3309) Brorfelde & 4.4 & 0.26 & 2.0 & $<0.08$ & 2.5042 & 18.46446 & 18.6 & 0.10 & 0.016 & 17 & 1.08 & 1.19 \\
\hline (9260) Edwardolson & 3.9 & 0.27 & 2.0 & & 3.0854 & 17.785 & 17.75 & 0.12 & 0.022 & 10 & 1.10 & 1.28 \\
\hline (17260) $2000 \mathrm{JQ58}$ & 3.3 & 0.26 & 1.8 & $<0.20$ & 3.1287 & 14.75761 & 14.74 & 0.14 & 0.034 & 8 & 1.13 & 1.54 \\
\hline (80218) 1999 VO123 & 0.88 & 0.32 & 3.1 & $<0.20$ & 3.1451 & 33.10 & 33.4 & 0.20 & 0.044 & 3 & 1.21 & 1.52 \\
\hline \multicolumn{13}{|c|}{ Candidate synchronous secondaries: } \\
\hline (1453) Fennia & 6.33 & 0.28 & 2.6 & $<0.03$ & 4.4121 & 23.00351 & 23.1 & 0.17 & 0.016 & 14 & 1.14 & 1.17 \\
\hline (5407) $1992 \mathrm{AX}$ & 3.7 & 0.22 & 1.7 & $<0.11$ & 2.5488 & 13.51783 & 13.52 & 0.12 & 0.025 & 10 & 1.10 & 1.52 \\
\hline (8306) Shoko & 2.4 & 0.45 & 3.3 & & 3.3503 & 36.20 & 36.20 & 0.11 & 0.05 & 8 & 1.11 & 1.27 \\
\hline (16635) $1993 \mathrm{QO}$ & 3.6 & 0.35 & 3.0 & & 2.2083 & 32.25 & 32.25 & 0.18 & 0.031 & 18 & 1.14 & 1.20 \\
\hline (136617) $1994 \mathrm{CC}$ & 0.62 & 0.18 & 2.8 & $<0.05$ & 2.3886 & 29.8 & 26 & & & & 1.03 & \\
\hline (276049) 2002 CE26 & 3.45 & 0.09 & 1.36 & $<0.06$ & 3.2930 & 15.6 & $\sim 15$ & & & & 1.01 & \\
\hline
\end{tabular}

Note: The values in the fifth column are $3-\sigma$ upper limits on eccentricity. 
Table 2

Synchronous secondary rotational lightcurves

\begin{tabular}{|c|c|c|c|c|c|c|c|c|c|c|}
\hline Binary system & Epoch JD & $\begin{array}{l}P_{2} \\
\text { (h) }\end{array}$ & $\begin{array}{l}\text { Four. } \\
\text { orders }\end{array}$ & $\begin{array}{r}\text { Mean } \\
\text { SolPh } \\
\left({ }^{\circ}\right)\end{array}$ & $\begin{array}{c}A_{2} \\
\text { (mag) }\end{array}$ & $\begin{array}{l}\text { Phase } \\
\text { Event }\end{array}$ & $\begin{array}{l}\text { Phase } \\
\text { P2min }\end{array}$ & $\begin{array}{c}\text { Phase } \\
\text { Diff. }\end{array}$ & $\begin{array}{l}\text { M.A. } \\
\text { Offset } \\
\left({ }^{\circ}\right)\end{array}$ & Fig. \\
\hline (2121) Sevastopol & 2455416.24500 & 37.130 & $1-4$ & 22 & 0.076 & 0.248 & 0.255 & 0.007 & 2.7 & $3 a$ \\
\hline (2121) Sevastopol & 2455962.12000 & 37.130 & 2,4 & 2 & 0.048 & 0.250 & 0.257 & 0.007 & 2.7 & $3 \mathrm{~b}$ \\
\hline (2121) Sevastopol & 2456513.28501 & 37.130 & 2 & 11 & 0.082 & 0.250 & 0.264 & 0.015 & 5.3 & $3 \mathrm{c}$ \\
\hline (2131) Mayall & 2455173.60250 & 23.470 & 2 & 22 & 0.035 & 0.253 & 0.247 & -0.006 & -2.3 & 5 \\
\hline (5477) Holmes & 2454255.70000 & 24.420 & 2 & 13 & 0.052 & 0.243 & 0.230 & -0.012 & -4.4 & $7 \mathrm{a}$ \\
\hline (5477) Holmes & 2456003.42867 & 24.420 & 2 & 16 & 0.048 & 0.243 & 0.279 & 0.036 & 13.0 & $7 \mathrm{~b}$ \\
\hline (5477) Holmes & 2456620.29000 & 24.420 & 2 & 8 & 0.053 & 0.255 & 0.274 & 0.019 & 6.8 & $7 \mathrm{c}$ \\
\hline (5481) Kiuchi & 2454557.57361 & 20.904 & 2 & 10 & 0.022 & 0.239 & 0.254 & 0.016 & 5.6 & $9 \mathrm{a}$ \\
\hline (5481) Kiuchi & 2456570.15250 & 20.904 & 1,2 & 7 & 0.033 & 0.256 & 0.228 & -0.028 & -9.9 & $9 \mathrm{~b}$ \\
\hline (5481) Kiuchi & 2457038.48700 & 20.904 & 2 & 16 & 0.033 & 0.248 & 0.269 & 0.022 & 7.7 & $10 \mathrm{a}$ \\
\hline (5481) Kiuchi & 2457067.66550 & 20.904 & 2 & 2 & 0.039 & 0.268 & 0.124 & -0.143 & -51.5 & $10 \mathrm{~b}$ \\
\hline (5481) Kiuchi & 2457099.46900 & 20.904 & 1,2 & 14 & 0.052 & 0.250 & 0.263 & 0.013 & 4.7 & $10 \mathrm{c}$ \\
\hline (5905) Johnson & 2453469.62542 & 21.780 & 2 & 20 & 0.026 & 0.258 & 0.267 & 0.009 & 3.4 & $12 \mathrm{a}$ \\
\hline (5905) Johnson & 2455809.22813 & 21.780 & 2 & 19 & 0.035 & 0.249 & 0.198 & -0.051 & -18.5 & $12 \mathrm{~b}$ \\
\hline (5905) Johnson & 2455829.19313 & 21.780 & 2 & 20 & 0.046 & 0.255 & 0.251 & -0.004 & -1.5 & $12 \mathrm{c}$ \\
\hline (5905) Johnson & 2455843.71313 & 21.780 & 2 & 24 & 0.035 & 0.252 & 0.229 & -0.024 & -8.5 & $13 \mathrm{a}$ \\
\hline (5905) Johnson & 2455852.78813 & 21.780 & 2 & 27 & 0.038 & 0.259 & 0.246 & -0.013 & -4.7 & $13 \mathrm{~b}$ \\
\hline (5905) Johnson & 2456384.05500 & 21.780 & 2 & 20 & 0.034 & 0.250 & 0.296 & 0.046 & 16.5 & $14 \mathrm{a}$ \\
\hline (5905) Johnson & 2456421.71625 & 21.780 & 2 & 24 & 0.034 & 0.253 & 0.290 & 0.036 & 13.1 & $14 \mathrm{~b}$ \\
\hline (7088) Ishtar & 2453764.52792 & 20.630 & $1-6$ & 20 & 0.090 & 0.251 & 0.282 & 0.031 & 11.1 & 16 \\
\hline (44620) 1999 RS43 & 2456747.94366 & 33.630 & 1,2 & 9 & 0.037 & 0.265 & 0.270 & 0.006 & 2.0 & 18 \\
\hline (66063) $1998 \mathrm{RO} 1$ & 2452903.91083 & 14.540 & $1-4$ & 17 & 0.118 & 0.259 & 0.272 & 0.014 & 5.0 & $20 \mathrm{a}$ \\
\hline (66063) 1998 RO1 & 2453263.33182 & 14.540 & $1-4$ & 31 & 0.120 & 0.259 & 0.258 & -0.001 & -0.3 & $20 \mathrm{~b}$ \\
\hline (76818) 2000 RG79 & 2453609.22150 & 14.127 & $1-4$ & 16 & 0.055 & 0.252 & 0.254 & 0.003 & 0.9 & $22 \mathrm{a}$ \\
\hline (76818) 2000 RG79 & 2456518.23175 & 14.127 & 1,2 & 15 & 0.049 & 0.256 & 0.284 & 0.028 & 10.2 & $22 \mathrm{a}$ \\
\hline (85938) 1999 DJ4 & 2453083.99000 & 17.730 & 2 & 67 & 0.130 & 0.242 & 0.210 & -0.032 & -11.3 & 24 \\
\hline (175706) 1996 FG3 & 2450188.89967 & 16.150 & 2 & 41 & 0.039 & 0.246 & 0.210 & -0.035 & -12.8 & $26 \mathrm{a}$ \\
\hline (175706) 1996 FG3 & 2451161.84583 & 16.150 & $1-3$ & 15 & 0.030 & 0.221 & 0.250 & 0.029 & 10.6 & $26 \mathrm{~b}$ \\
\hline (175706) 1996 FG3 & 2455597.82000 & 16.150 & 2 & 9 & 0.015 & 0.240 & 0.191 & -0.048 & -17.4 & $27 \mathrm{a}$ \\
\hline (175706) 1996 FG3 & 2455919.56792 & 16.150 & 2 & 11 & 0.024 & 0.240 & 0.226 & -0.014 & -5.1 & $27 \mathrm{~b}$ \\
\hline (399774) 2005 NB7 & 2454592.72000 & 15.280 & $1-4$ & 58 & 0.094 & 0.256 & 0.270 & 0.015 & 5.3 & 29 \\
\hline (3309) Brorfelde & 2455479.78694 & 18.454 & 1,2 & 17 & 0.016 & 0.253 & 0.241 & -0.011 & -4.1 & $31 \mathrm{a}$ \\
\hline (3309) Brorfelde & 2455504.40500 & 18.454 & 2 & 7 & 0.012 & 0.252 & 0.232 & -0.020 & -7.2 & $31 b$ \\
\hline (3309) Brorfelde & 2456112.06470 & 18.454 & 2 & 26 & 0.020 & 0.236 & 0.249 & 0.013 & 4.6 & $31 \mathrm{c}$ \\
\hline (9260) Edwardolson & 2453662.77500 & 17.785 & 2 & 10 & 0.023 & 0.249 & 0.239 & -0.009 & -3.4 & $33 \mathrm{a}$ \\
\hline (9260) Edwardolson & 2456212.27750 & 17.785 & 2 & 14 & 0.020 & 0.253 & 0.275 & 0.023 & 8.1 & $33 \mathrm{~b}$ \\
\hline (17260) $2000 \mathrm{JQ58}$ & 2453783.42375 & 14.757 & 2 & 10 & 0.046 & 0.248 & 0.270 & 0.022 & 8.0 & $35 a$ \\
\hline (17260) 2000 JQ58 & 2455847.86362 & 14.757 & 2 & 5 & 0.023 & 0.236 & 0.214 & -0.022 & -7.9 & $35 b$ \\
\hline (80218) 1999 VO123 & 2456218.42241 & 33.100 & 2 & 2 & 0.055 & 0.260 & 0.236 & -0.024 & -8.5 & $37 \mathrm{a}$ \\
\hline (80218) 1999 VO123 & 2456721.75857 & 33.100 & 2 & 3 & 0.033 & 0.254 & 0.143 & -0.111 & -40.1 & $37 \mathrm{~b}$ \\
\hline (1453) Fennia & 2455596.82286 & 23.000 & 2 & 17 & 0.010 & 0.255 & 0.247 & -0.008 & -3.0 & $39 a$ \\
\hline (1453) Fennia & 2456195.71583 & 23.000 & 2 & 16 & 0.018 & 0.247 & 0.224 & -0.024 & -8.5 & $39 b$ \\
\hline (1453) Fennia & 2456216.32000 & 23.000 & 2 & 8 & 0.020 & 0.255 & 0.218 & -0.037 & -13.2 & $39 \mathrm{c}$ \\
\hline (5407) $1992 \mathrm{AX}$ & 2450475.00000 & 13.518 & 1,2 & 10 & 0.028 & 0.246 & 0.252 & 0.006 & 2.1 & $40 \mathrm{a}$ \\
\hline (5407) $1992 \mathrm{AX}$ & 2454136.05300 & 13.518 & 1,2 & 12 & 0.033 & 0.249 & 0.298 & 0.049 & 17.5 & $40 \mathrm{~b}$ \\
\hline (5407) $1992 \mathrm{AX}$ & 2455965.46196 & 13.508 & $1-3$ & 8 & 0.023 & 0.254 & 0.277 & 0.023 & 8.3 & $41 \mathrm{a}$ \\
\hline (5407) $1992 \mathrm{AX}$ & 2456005.98596 & 13.508 & 1,2 & 32 & 0.017 & 0.260 & 0.307 & 0.046 & 16.7 & $41 \mathrm{~b}$ \\
\hline (5407) $1992 \mathrm{AX}$ & 2456036.37896 & 13.508 & 1,2 & 43 & 0.026 & 0.252 & 0.293 & 0.041 & 14.6 & $41 \mathrm{c}$ \\
\hline
\end{tabular}


Table 3

Asteroids with asynchronous secondaries

\begin{tabular}{|c|c|c|c|c|c|c|c|c|c|c|c|}
\hline Asteroid & $\begin{array}{c}D_{1} \\
(\mathrm{~km})\end{array}$ & $\frac{D_{s}}{D_{1}}$ & $\frac{a}{D_{1}}$ & $e$ & $\begin{array}{l}P_{1} \\
(\mathrm{~h})\end{array}$ & $\begin{array}{c}P_{\text {orb }} \\
(\mathrm{h})\end{array}$ & $\begin{array}{l}P_{s} \\
(\mathrm{~h})\end{array}$ & $s$ & $\begin{array}{l}A_{1} \\
(\mathrm{mag})\end{array}$ & $\begin{array}{c}A_{s} \\
(\mathrm{mag})\end{array}$ & $\begin{array}{r}\text { SolPh } \\
\left({ }^{\circ}\right)\end{array}$ \\
\hline \multicolumn{12}{|c|}{ Close binary systems with asynchronous satellites: } \\
\hline (35107) $1991 \mathrm{VH}$ & 1.2 & 0.38 & 2.7 & 0.05 & 2.624 & 32.7 & $(12.836)$ & 2 & 0.08 & 0.06 & 27 \\
\hline (162000) 1990 OS & 0.3 & 0.15 & 2.2 & & & 21 & $\leq 8$ & 2 & & & \\
\hline (164121) 2003 YT1 & 1.0 & 0.18 & 3.2 & & 2.343 & 36.7 & $\leq 6$ & 2 & & & \\
\hline (311066) $2004 \mathrm{DC}$ & 0.3 & 0.20 & 2.2 & 0.24 & $(2.6)$ & 23 & 7 & 2 & & & \\
\hline (363027) $1998 \mathrm{ST} 27$ & 0.8 & 0.15 & 5.6 & $\geq 0.3$ & $<3$ & $\approx 100$ & $<6$ & 2 & & & \\
\hline \multicolumn{12}{|c|}{ Triple systems with asynchronous outer satellites: } \\
\hline (136617) $1994 \mathrm{CC}$ & 0.62 & 0.13 & 9.9 & 0.19 & 2.3886 & 201 & $\sim 14$ & 3 & & & \\
\hline (153591) 2001 SN263 & 2.5 & 0.31 & 6.7 & $<0.04$ & 3.4256 & 149.4 & 13.43 & 3 & & & \\
\hline \multicolumn{12}{|c|}{ Candidate triple systems with asynchronous outer satellites: } \\
\hline (1830) Pogson & 8.0 & & & & 2.57003 & & 3.2626 & 3 & 0.11 & 0.03 & 8 \\
\hline (2006) Polonskaya & 4.7 & & & & 3.11809 & & 6.6593 & 3 & 0.09 & 0.08 & 7 \\
\hline (2577) Litva & 5.7 & 0.32 & $(66)$ & & 2.8129 & $(5136)$ & 5.6818 & 3 & 0.17 & 0.06 & 16 \\
\hline (8306) Shoko & 2.4 & & & & 3.3503 & & $\sim 61$ & 3 & 0.11 & 0.06 & 8 \\
\hline (16635) $1993 \mathrm{QO}$ & 3.6 & & & & 2.2083 & & 7.623 & 3 & 0.18 & 0.04 & 18 \\
\hline \multicolumn{12}{|c|}{ (Semi-)wide systems with asynchronous satellites: } \\
\hline (1509) Esclangona & 8.5 & 0.33 & $\geq 23$ & & 3.25283 & $\gtrsim 700$ & 6.6422 & 2 & 0.13 & 0.04 & 23 \\
\hline (2623) Zech & 6.8 & $\geq 0.29$ & 7.1 & & 2.7401 & 117.2 & 18.718 & 2 & 0.22 & 0.08 & 17 \\
\hline (32039) $2000 \mathrm{JO} 23$ & 2.6 & $\geq 0.58$ & 16 & & 6.598 & 360 & 11.099 & $(2)$ & 0.21 & 0.07 & 9 \\
\hline (1717) Arlon & 7.8 & & & & $(5.148)$ & & $(18.23)$ & 3 & 0.08 & 0.13 & 11 \\
\hline \multicolumn{12}{|c|}{ Asteroids with two rotational lightcurves: } \\
\hline (2486) Metsahovi & 6.9 & & & & 2.6402 & & 4.4518 & 2 & 0.04 & 0.12 & 14 \\
\hline (3982) Kastel' & 5.4 & & & & 5.8358 & & 8.4865 & 2 & 0.08 & 0.27 & 10 \\
\hline (5474) Gingasen & 4.1 & & & & 3.1095 & & 3.6242 & 2 & 0.06 & 0.18 & 12 \\
\hline (11217) 1999 JC4 & 2.2 & & & & 4.8219 & & 9.584 & 2 & 0.10 & 0.08 & 26 \\
\hline (13123) Tyson & 8 & & & & 3.3303 & & 3.862 & 2 & 0.20 & 0.04 & 11 \\
\hline (114319) 2002 XD58 & 1.7 & & & & 2.9649 & & 7.954 & 2 & 0.14 & 0.09 & 17 \\
\hline
\end{tabular}


Table 4

Observed vs simulated secondary libration amplitudes

\begin{tabular}{lcc}
\hline Binary system & $\begin{array}{c}\text { Max. observed } \\
\text { offset } \mid \\
\left({ }^{\circ}\right)\end{array}$ & $\begin{array}{c}\text { Max. simulated } \\
\text { libration amplitude } \\
\left({ }^{\circ}\right)\end{array}$ \\
\hline (2121) Sevastopol & 5.3 & 22 \\
(2131) Mayall & 2.3 & 59 \\
(5477) Holmes & 13.0 & 20 \\
(5481) Kiuchi & 51.5 & 44 \\
(5905) Johnson & 18.5 & 12 \\
(7088) Ishtar & 11.1 & $>90$ \\
(66063) 1998 RO1 & 5.0 & 27 \\
(66391) 1999 KW4 & & 2 \\
(76818) 2000 RG79 & 10.2 & 48 \\
(175706) 1996 FG3 & 17.4 & 24 \\
(185851) 2000 DP107 & & 11 \\
(3309) Brorfelde & 7.2 & 18 \\
(17260) 2000 JQ58 & 8.0 & $>90$ \\
(80218) 1999 VO123 & 40.1 & $>90$ \\
(1453) Fennia & 13.2 & 61 \\
(5407) 1992 AX & 17.5 & \\
\hline
\end{tabular}




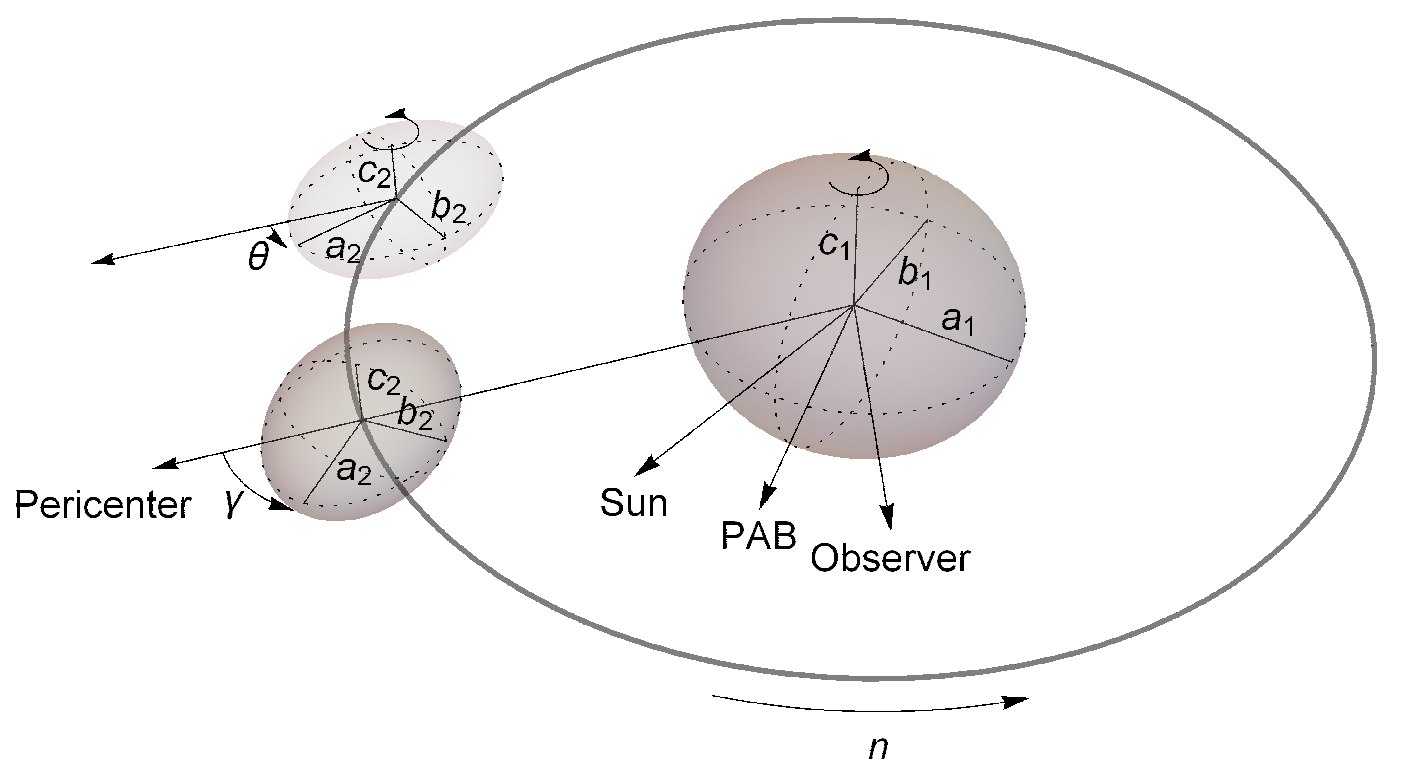

Fig. 1. Scheme of the binary geometry, illustrating the angular and axial parameters described in Sections 2 and 3. 


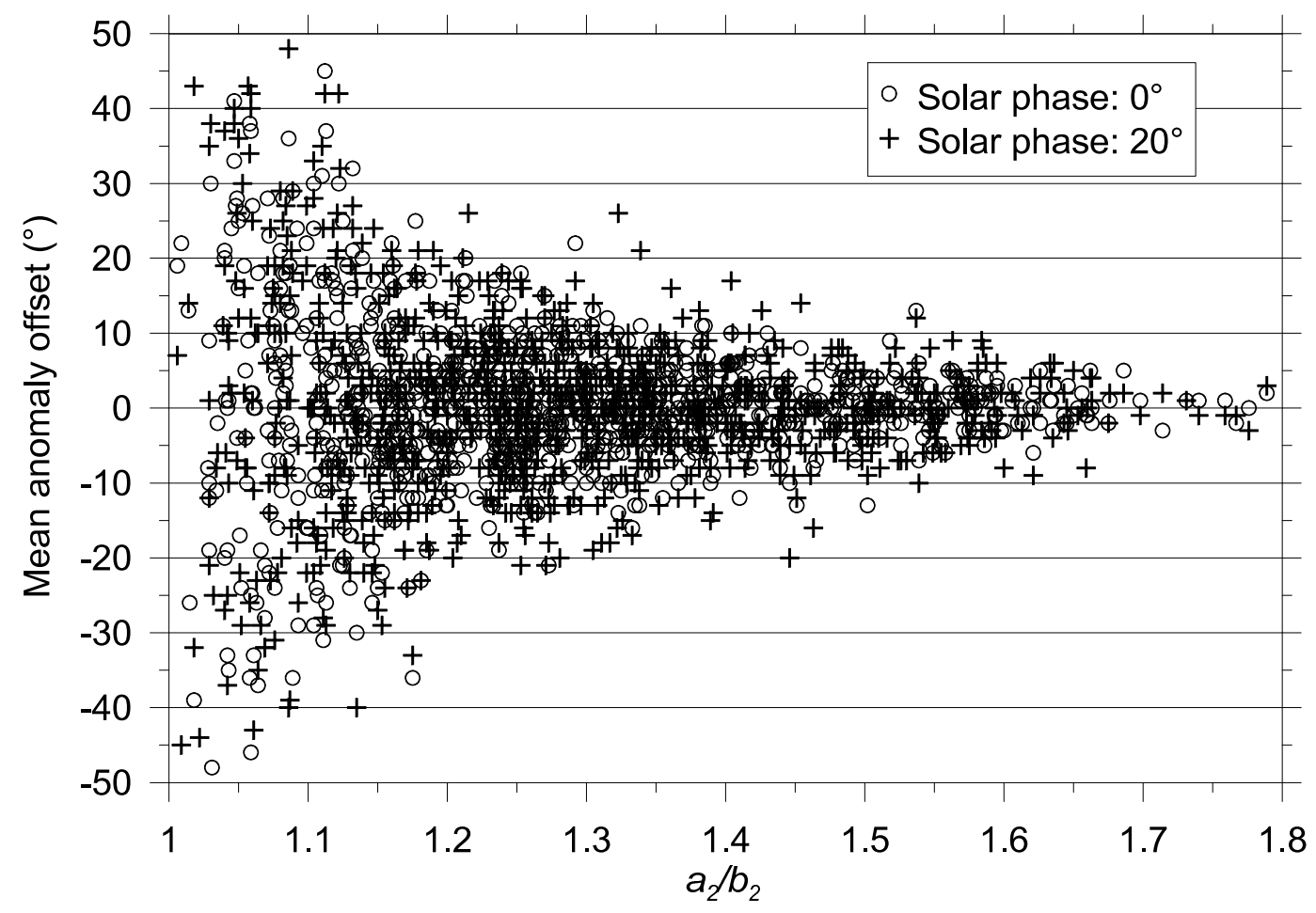

Fig. 2. Systematic offsets between the secondary rotational lightcurve minima and the mutual events vs the secondary equatorial axis ratio computed for 1000 simulated secondary shapes. 


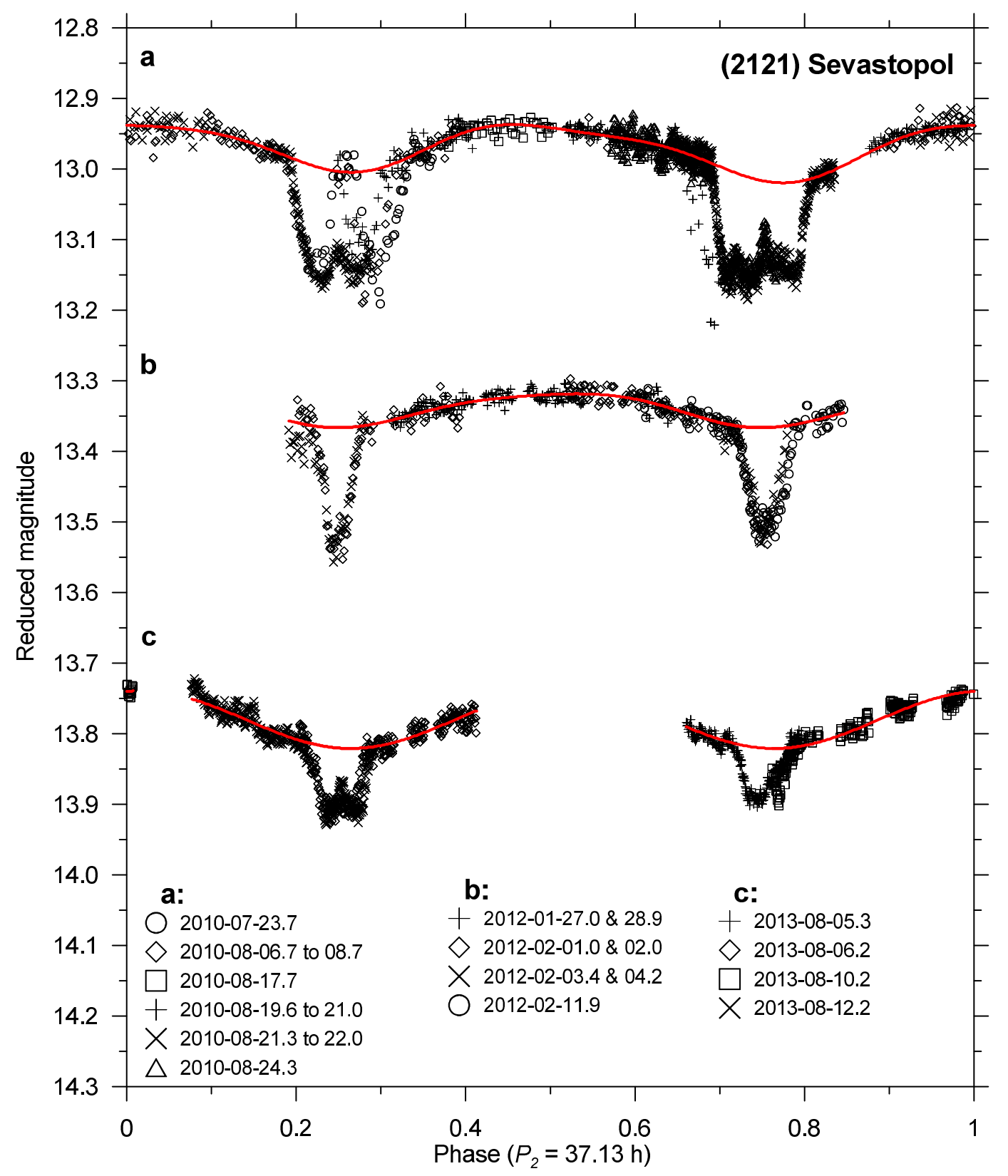

Fig. 3. Secondary lightcurves of (2121) Sevastopol.

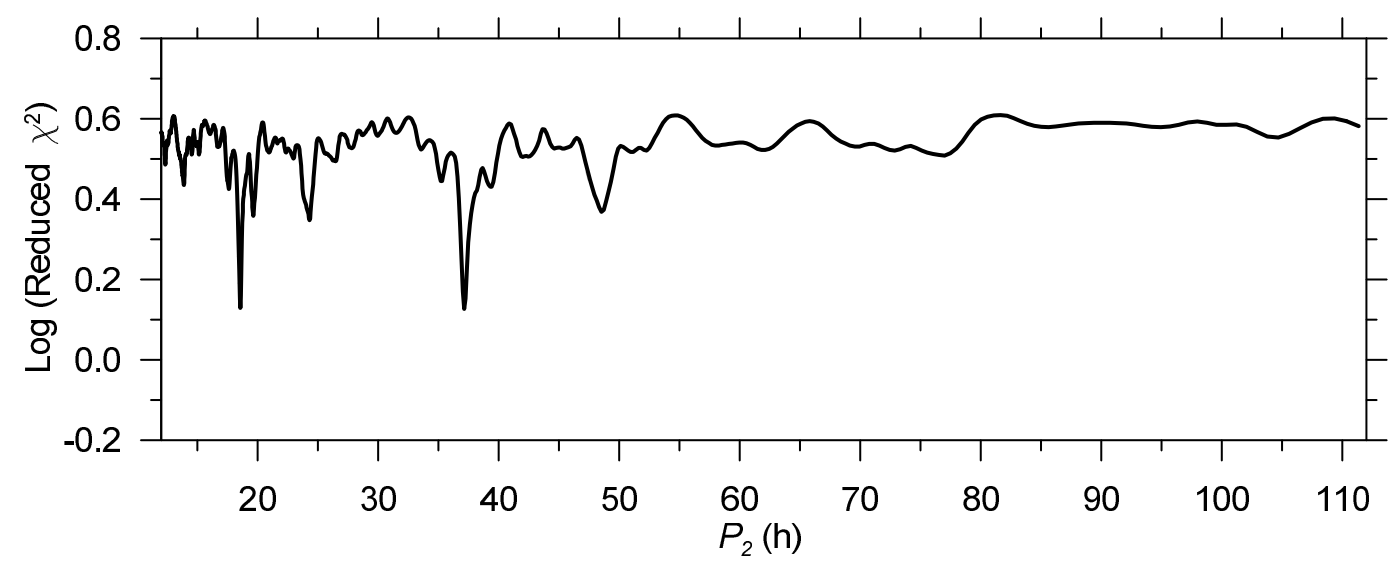

Fig. 4. Noise spectrum for the secondary rotational period of (2121) Sevastopol, the dataset for Epoch JD 2455416.245. 


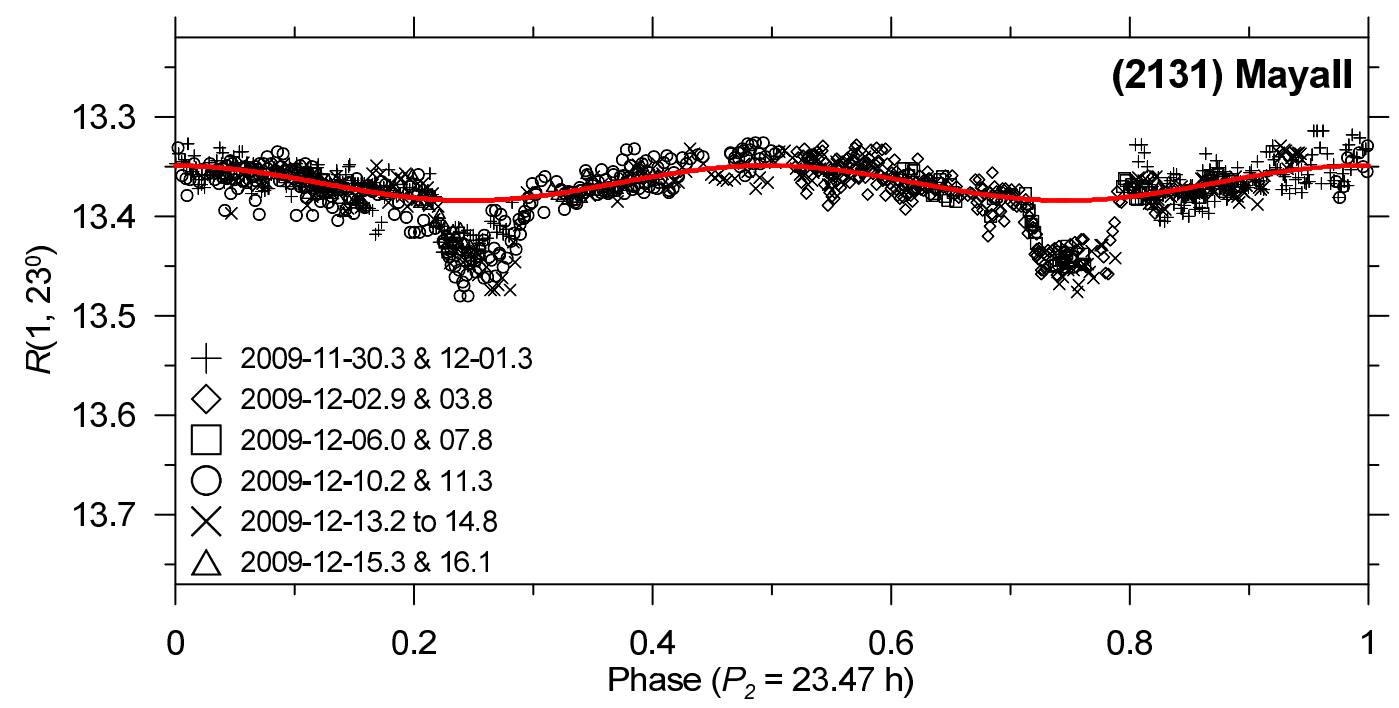

Fig. 5. Secondary lightcurve of (2131) Mayall.

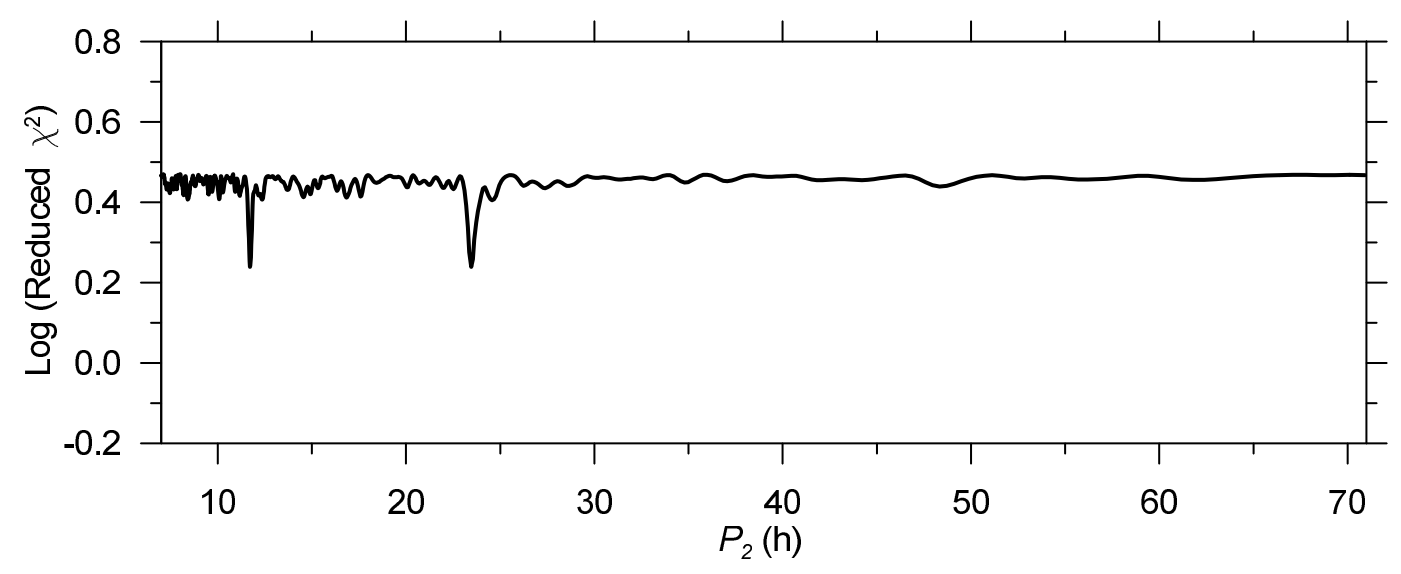

Fig. 6. Noise spectrum for the secondary rotational period of (2131) Mayall. 


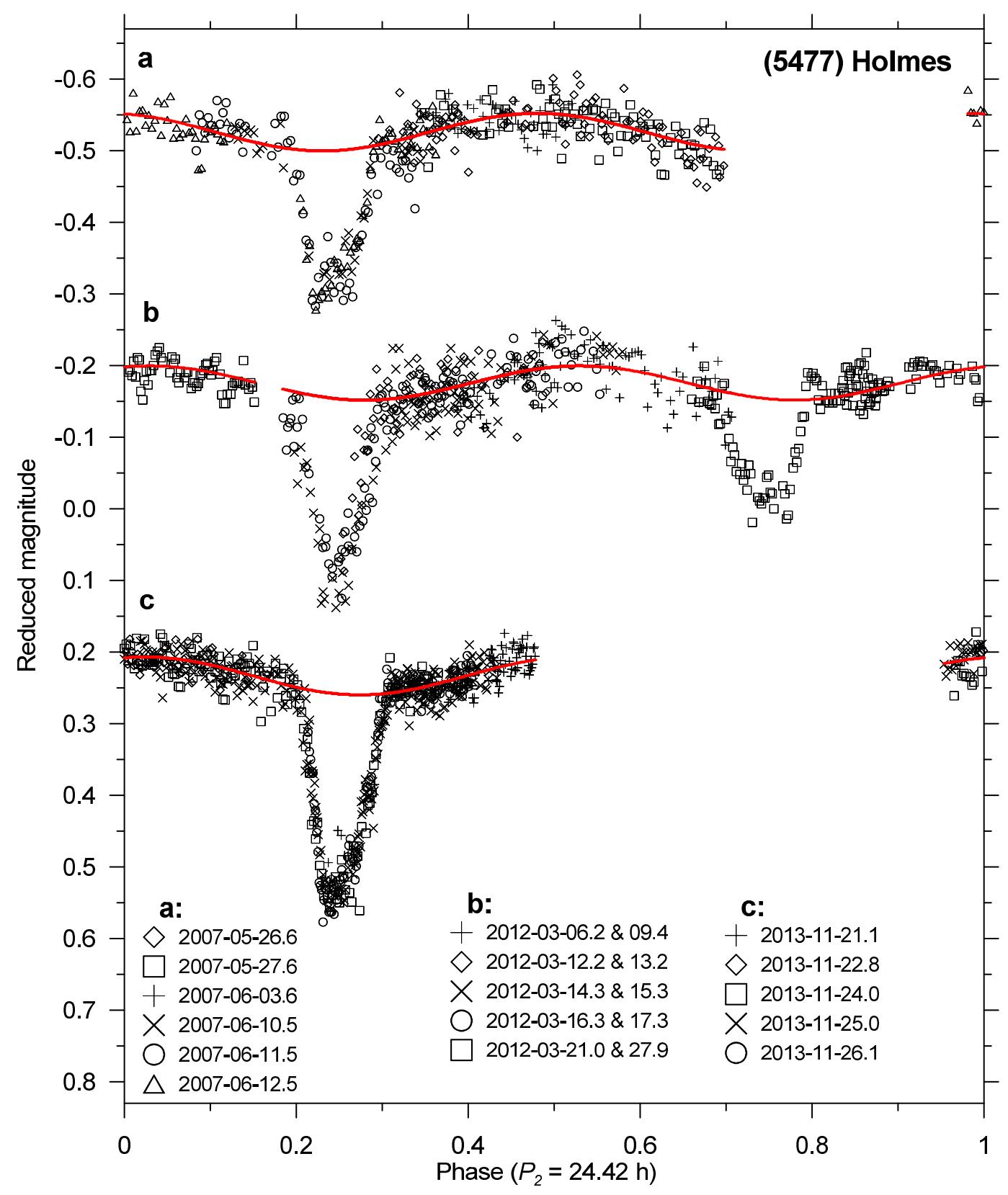

Fig. 7. Secondary lightcurves of (5477) Holmes.

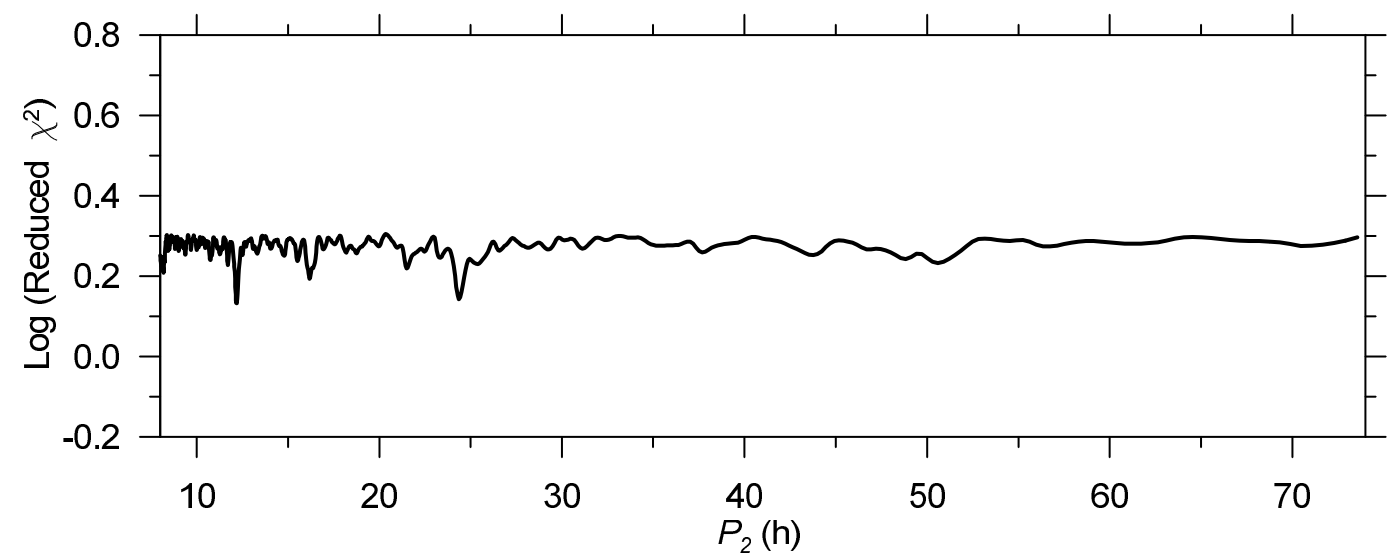

Fig. 8. Noise spectrum for the secondary rotational period of (5477) Holmes, the dataset for Epoch JD 2456003.42867. 


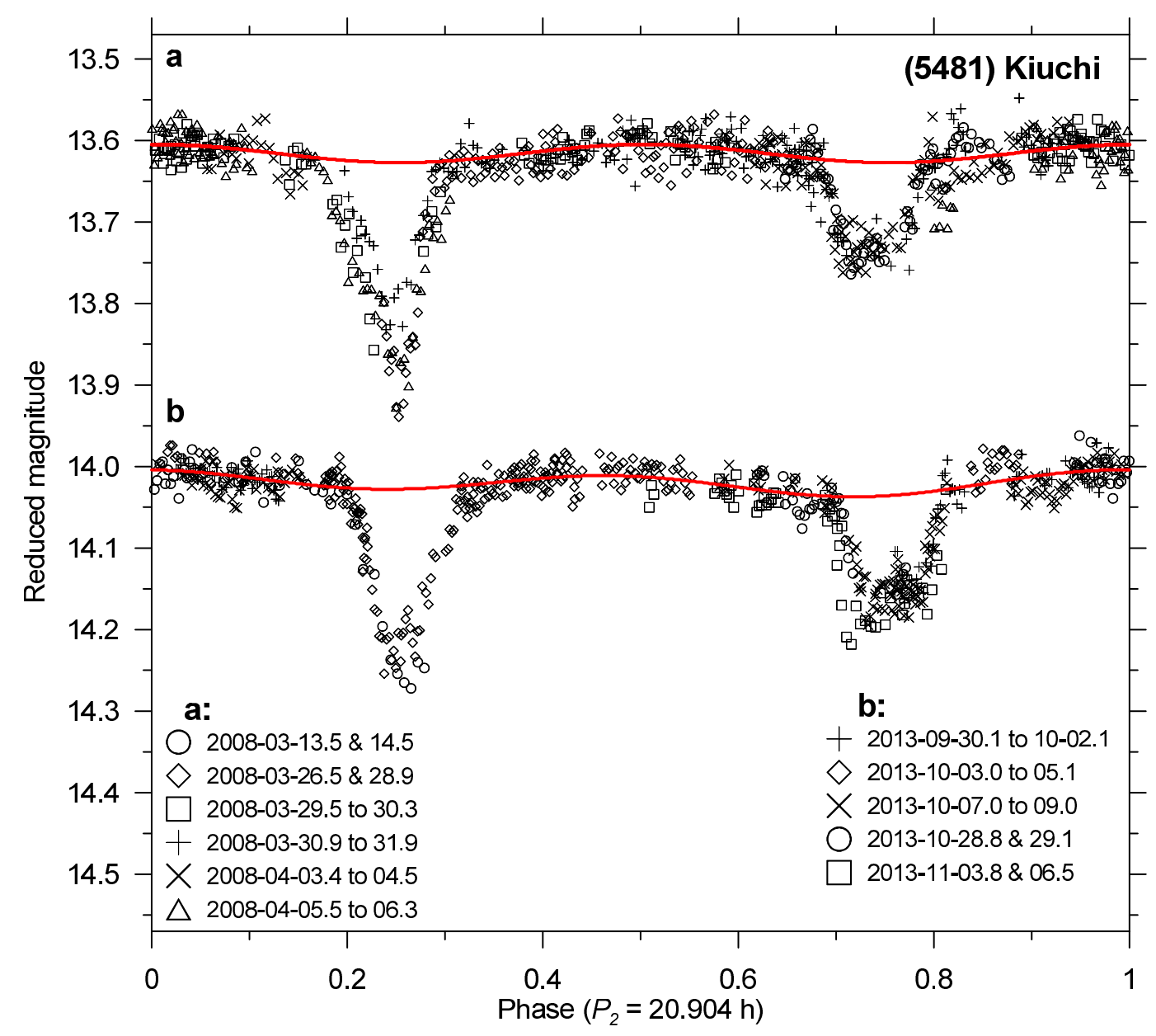

Fig. 9. Secondary lightcurves of (5481) Kiuchi from the 2008 and 2013 apparitions. 


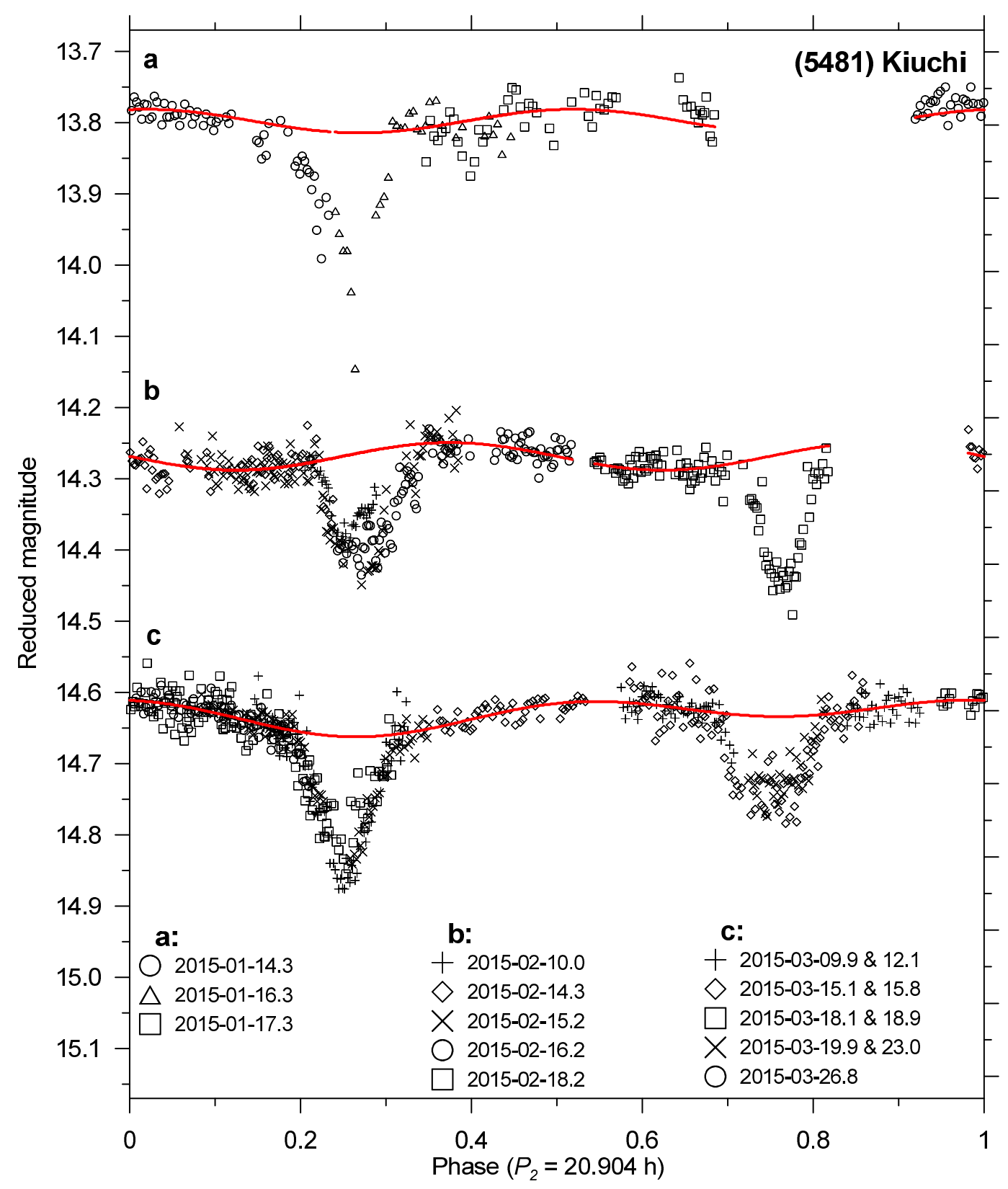

Fig. 10. Secondary lightcurves of (5481) Kiuchi from the 2015 apparition.

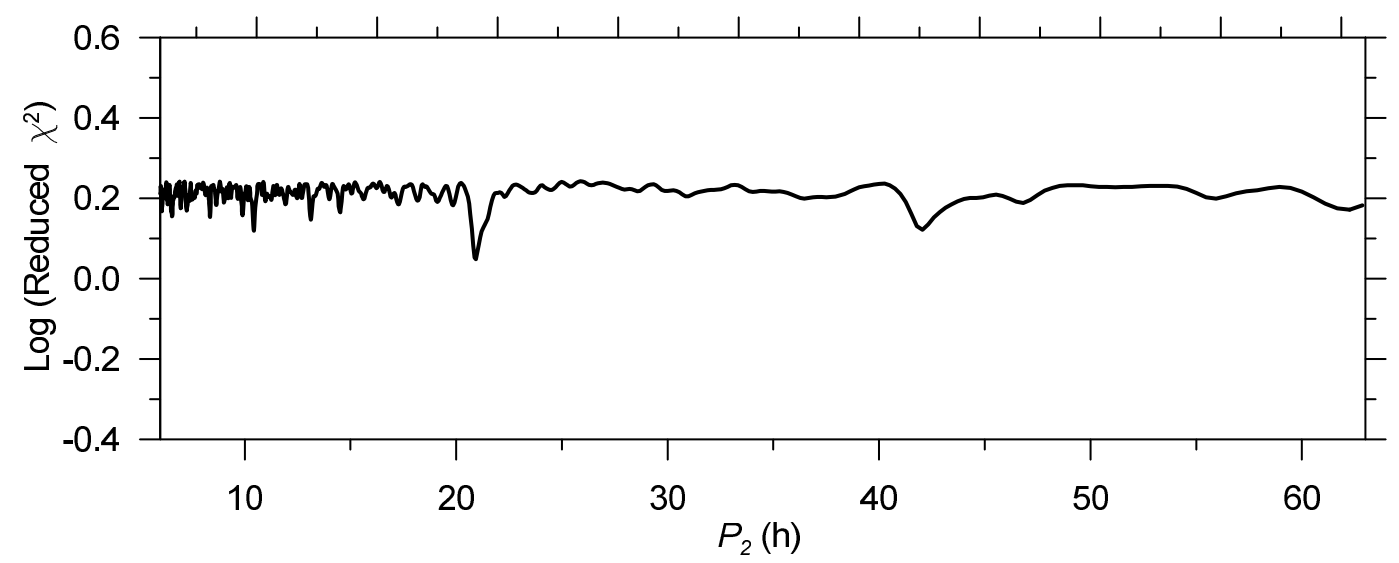

Fig. 11. Noise spectrum for the secondary rotational period of (5481) Kiuchi, the dataset for Epoch JD 2457099.469. 


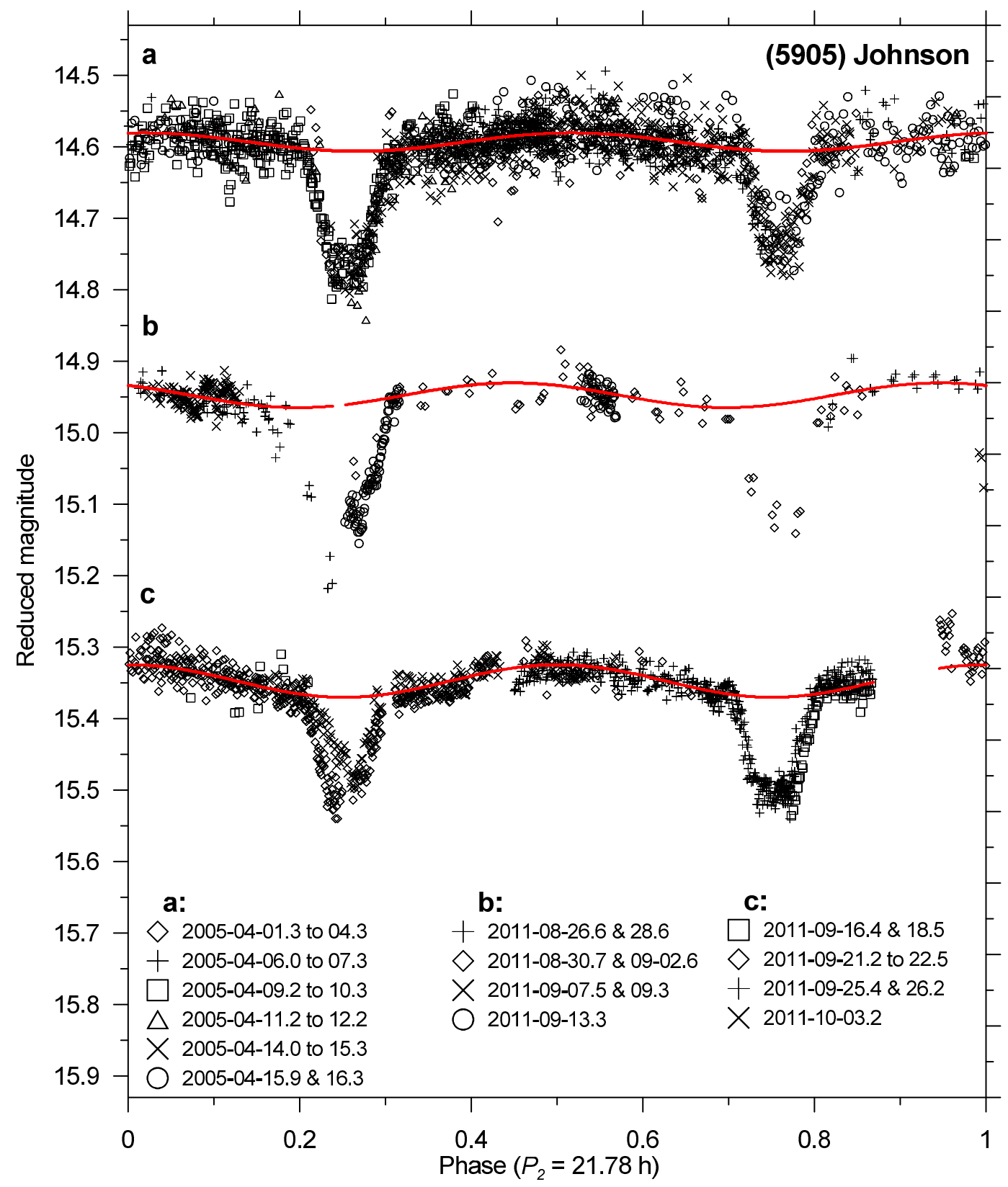

Fig. 12. Secondary lightcurves of (5905) Johnson from the 2005 and 2011 apparitions. 


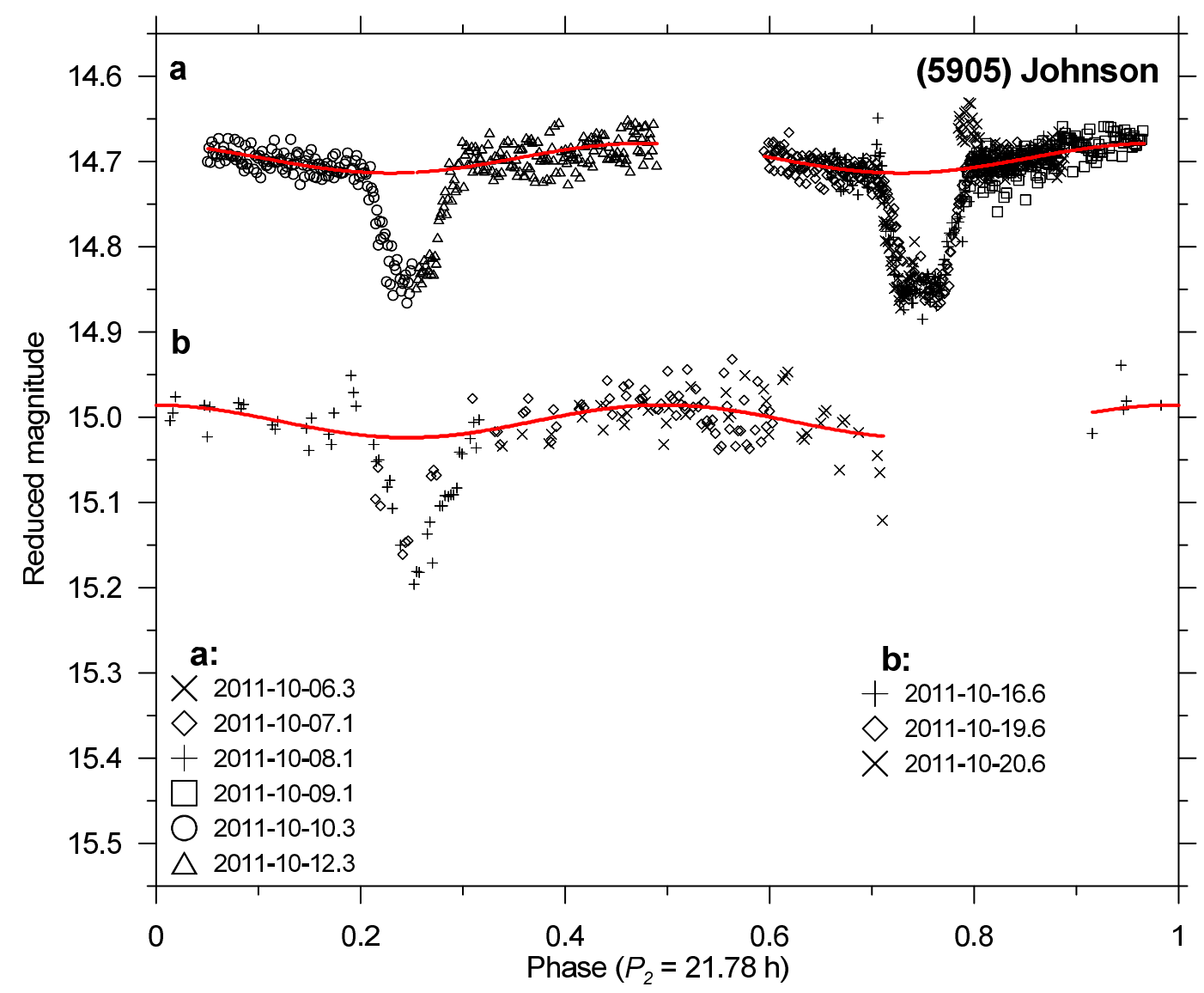

Fig. 13. Secondary lightcurves of (5905) Johnson from the 2011 apparition. 


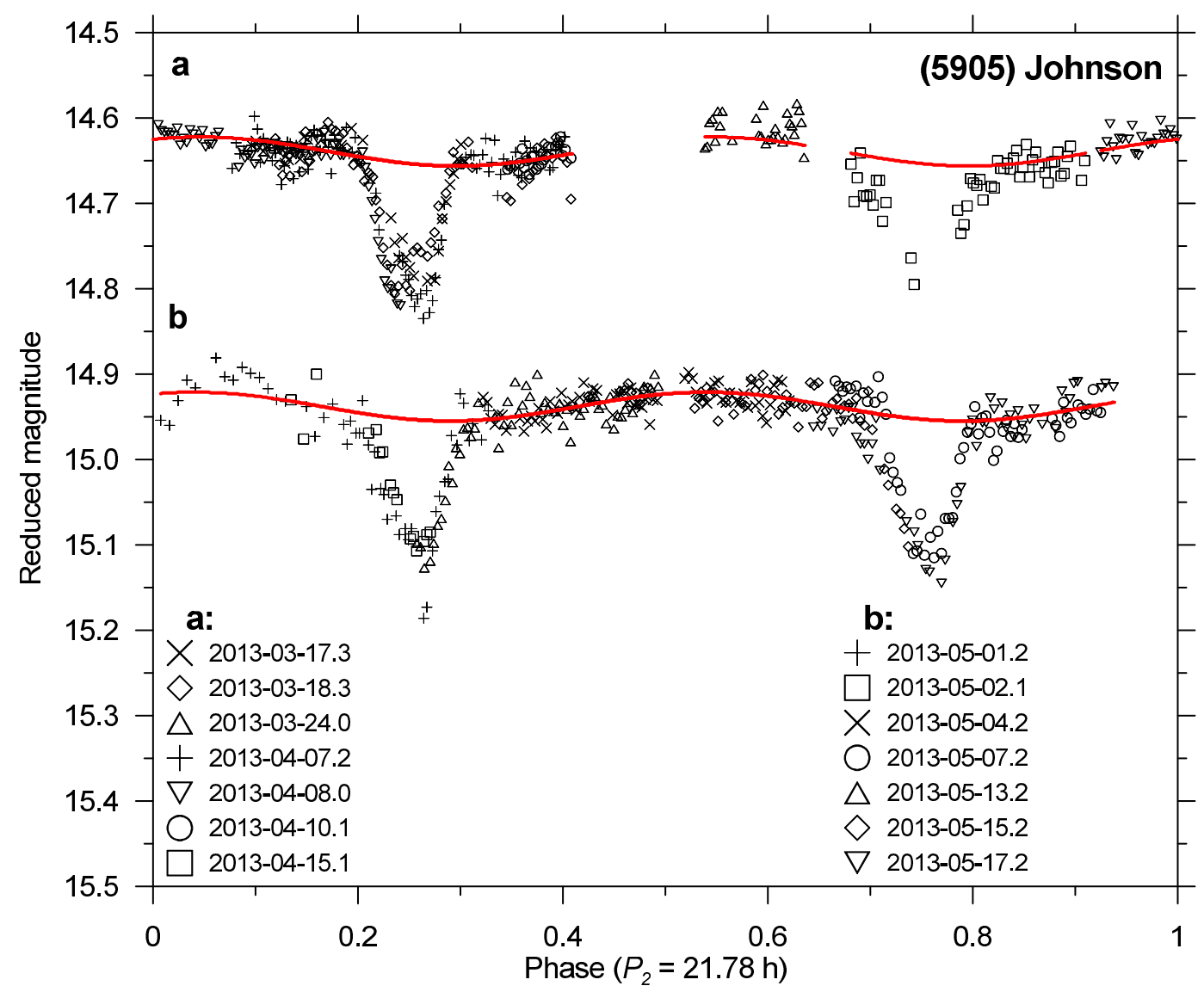

Fig. 14. Secondary lightcurves of (5905) Johnson from the 2013 apparition.

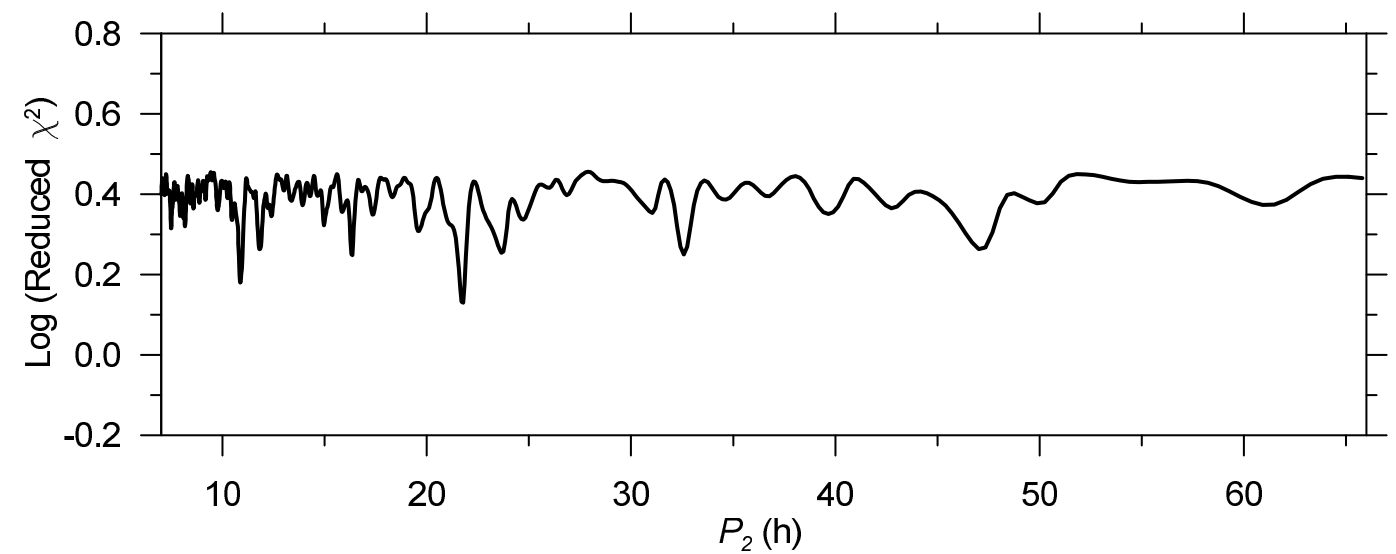

Fig. 15. Noise spectrum for the secondary rotational period of (5905) Johnson, the dataset for Epoch JD 2455843.71313. 


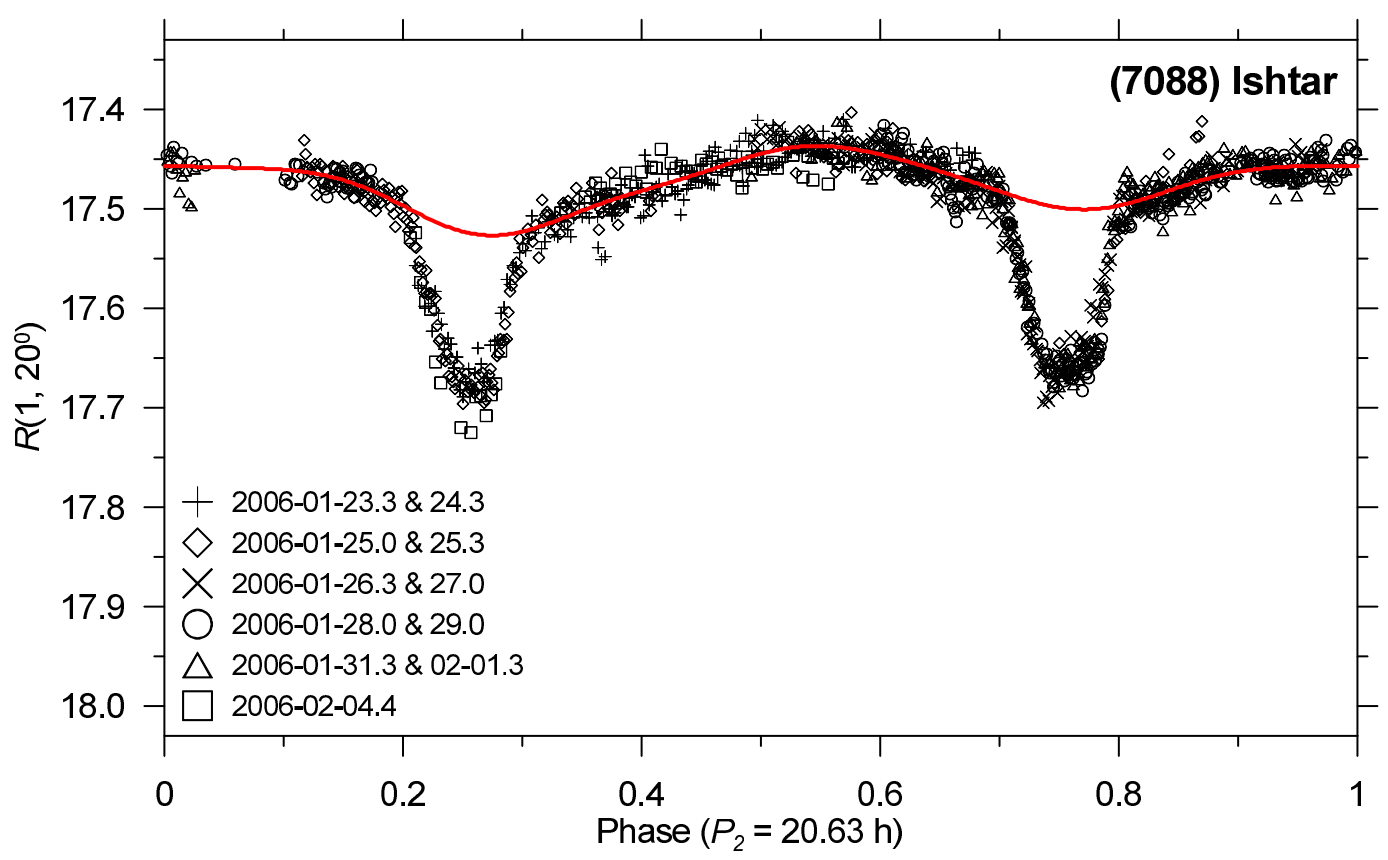

Fig. 16. Secondary lightcurve of (7088) Ishtar.

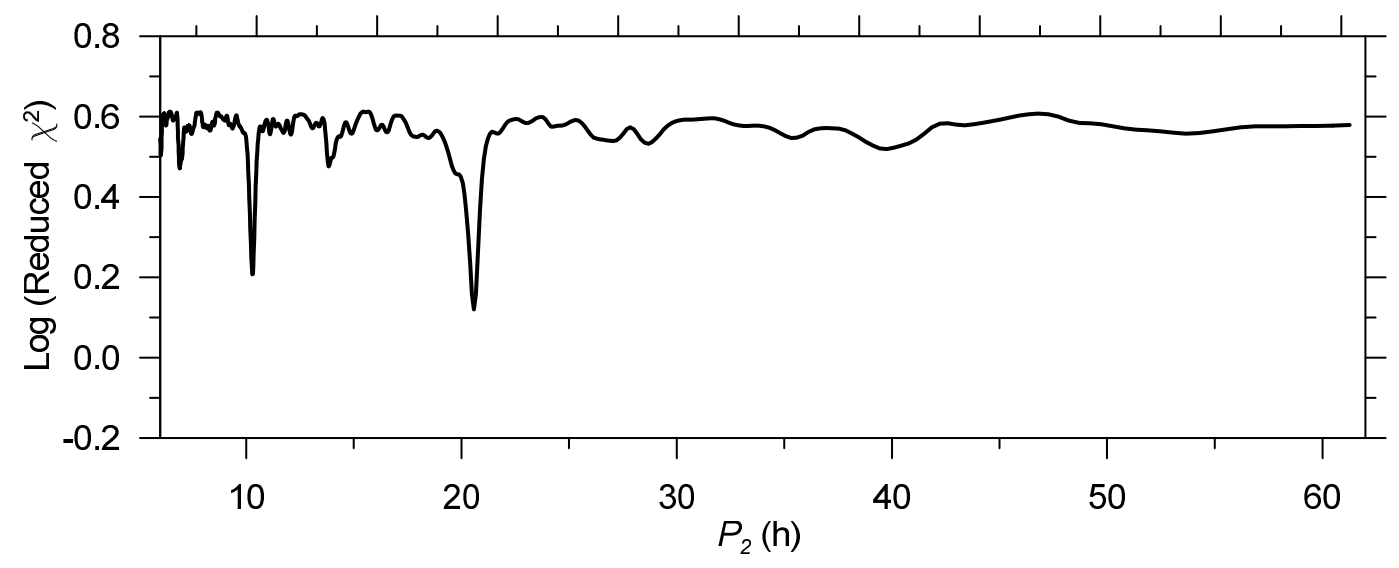

Fig. 17. Noise spectrum for the secondary rotational period of (7088) Ishtar. 


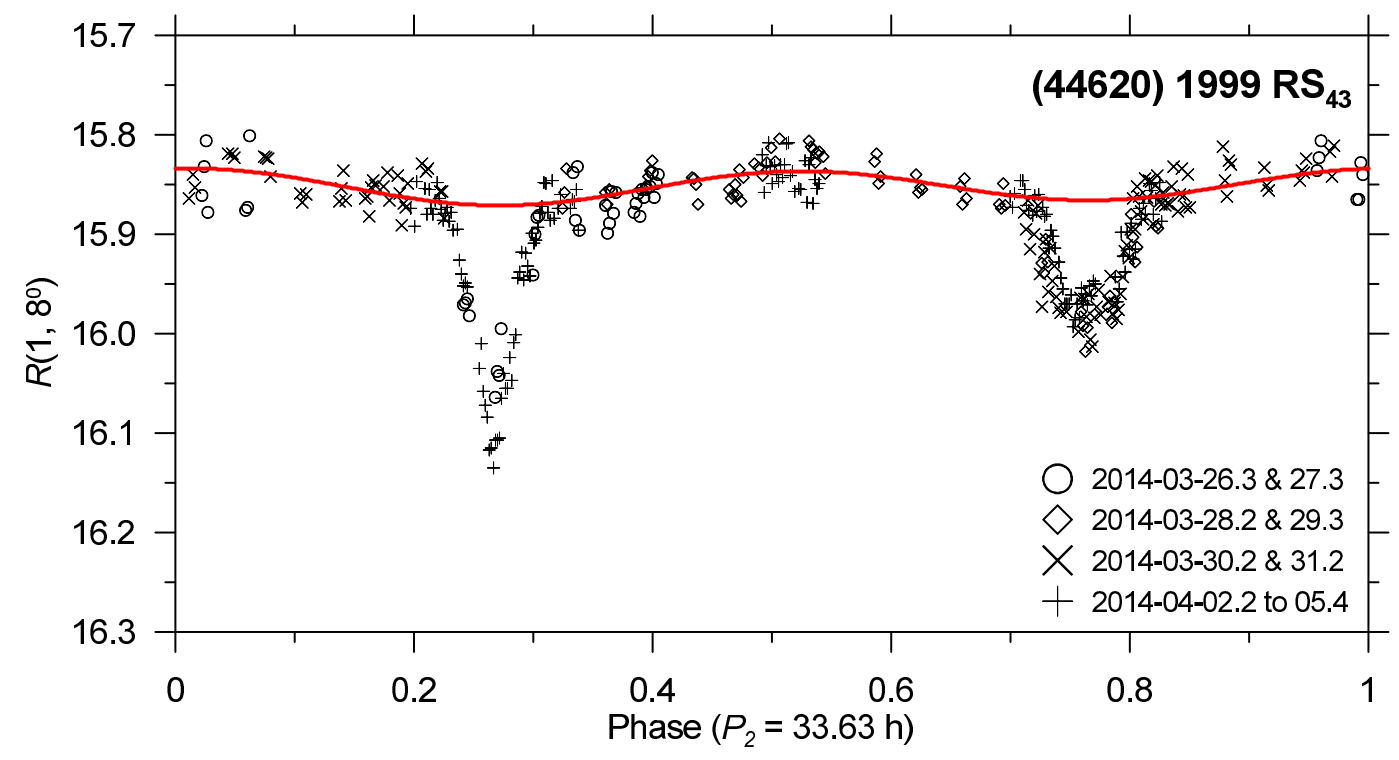

Fig. 18. Secondary lightcurve of (44620) 1999 RS43.

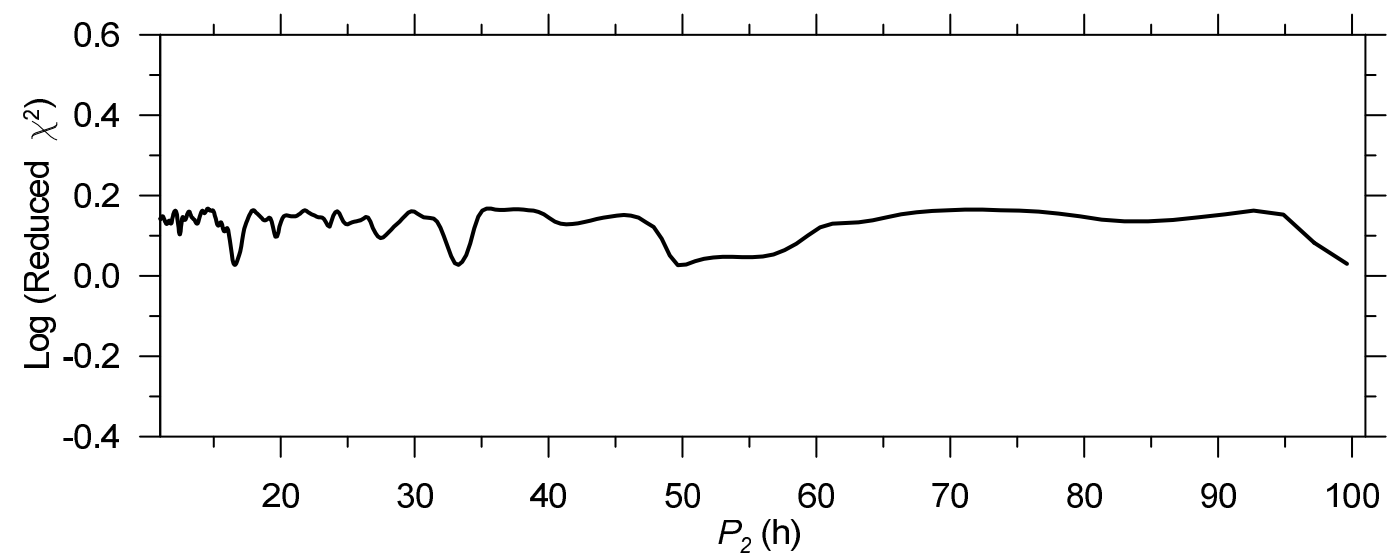

Fig. 19. Noise spectrum for the secondary rotational period of (44620) 1999 RS43. 


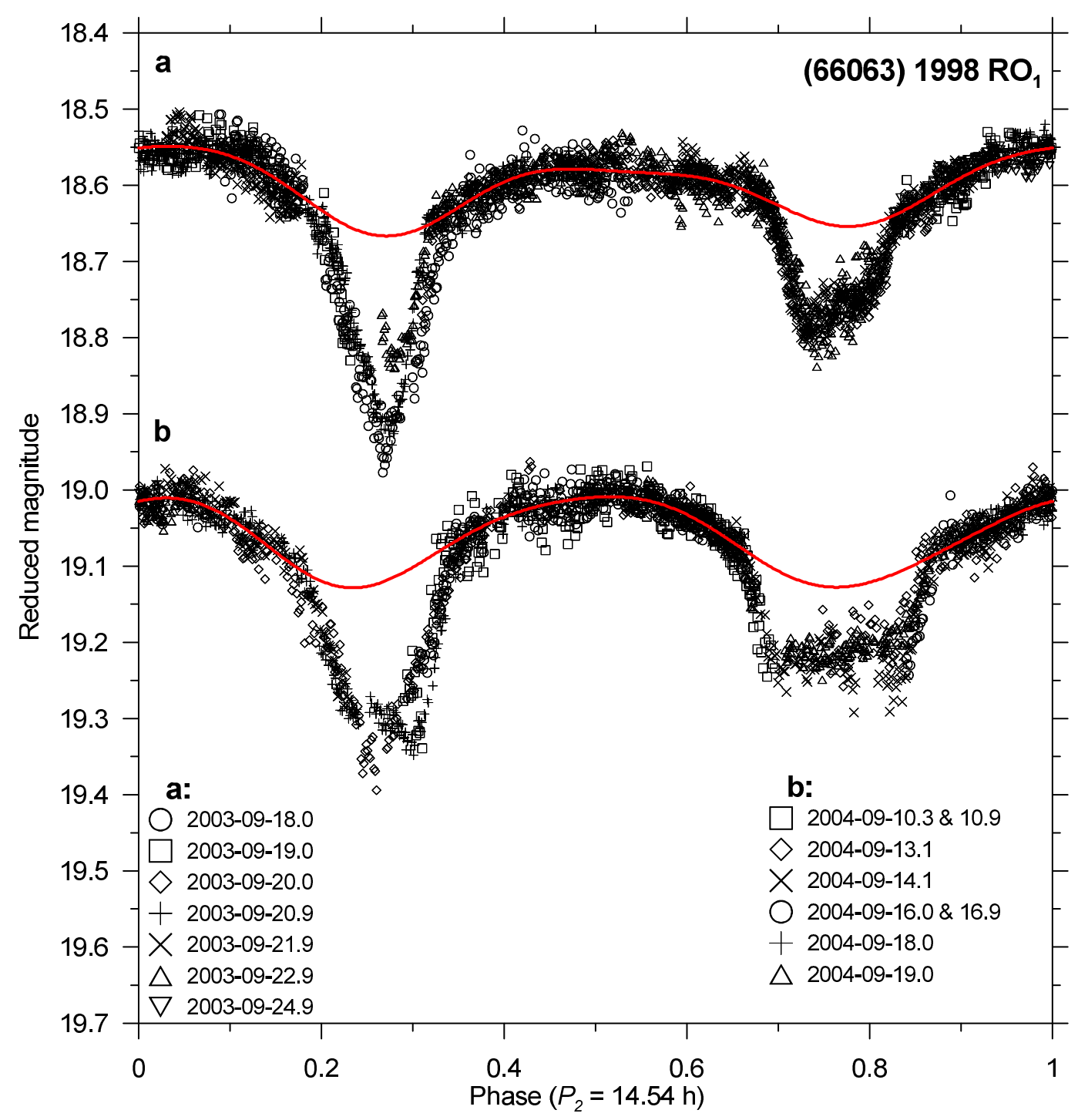

Fig. 20. Secondary lightcurves of (66063) 1998 RO1.

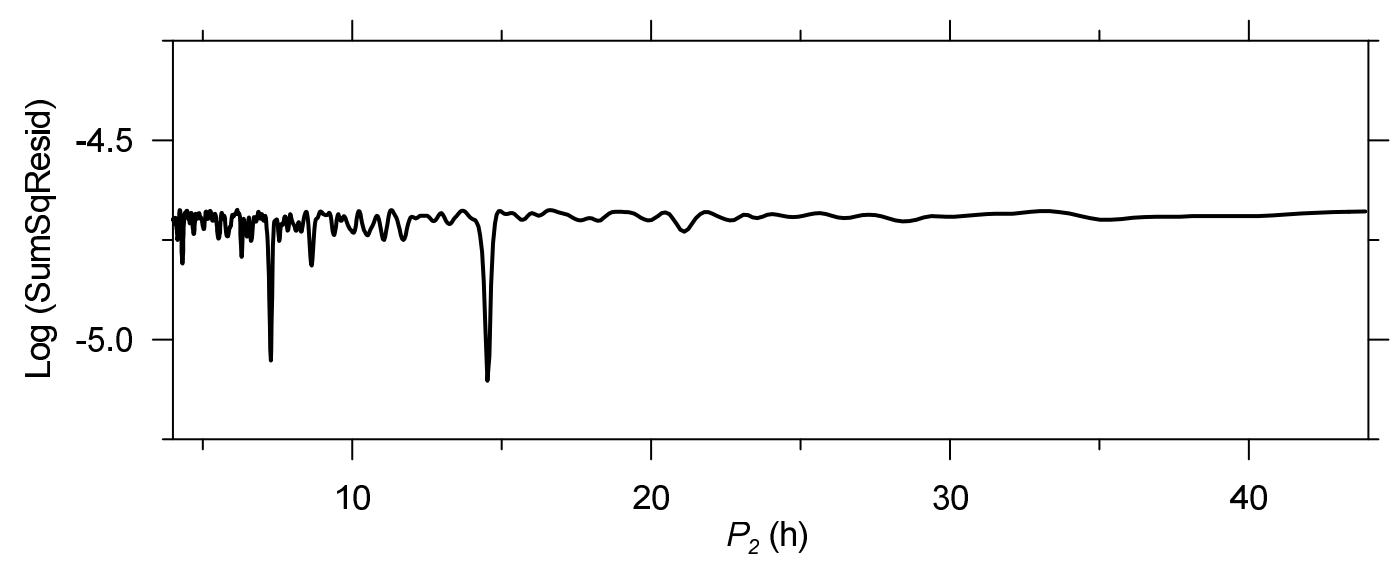

Fig. 21. Noise spectrum for the secondary rotational period of (66063) 1998 RO1, the dataset for Epoch JD 2453263.33182. 


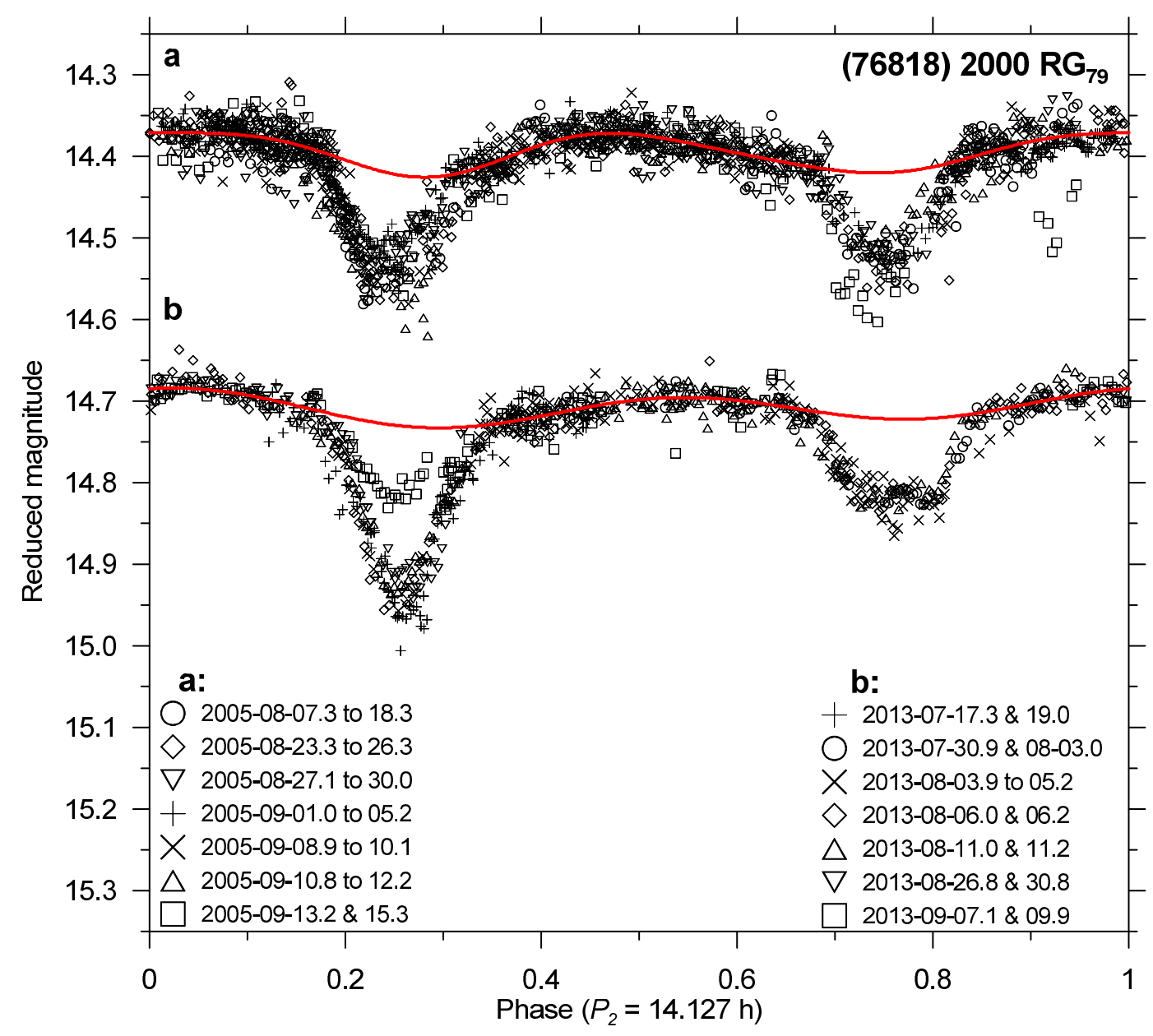

Fig. 22. Secondary lightcurves of (76818) 2000 RG79.

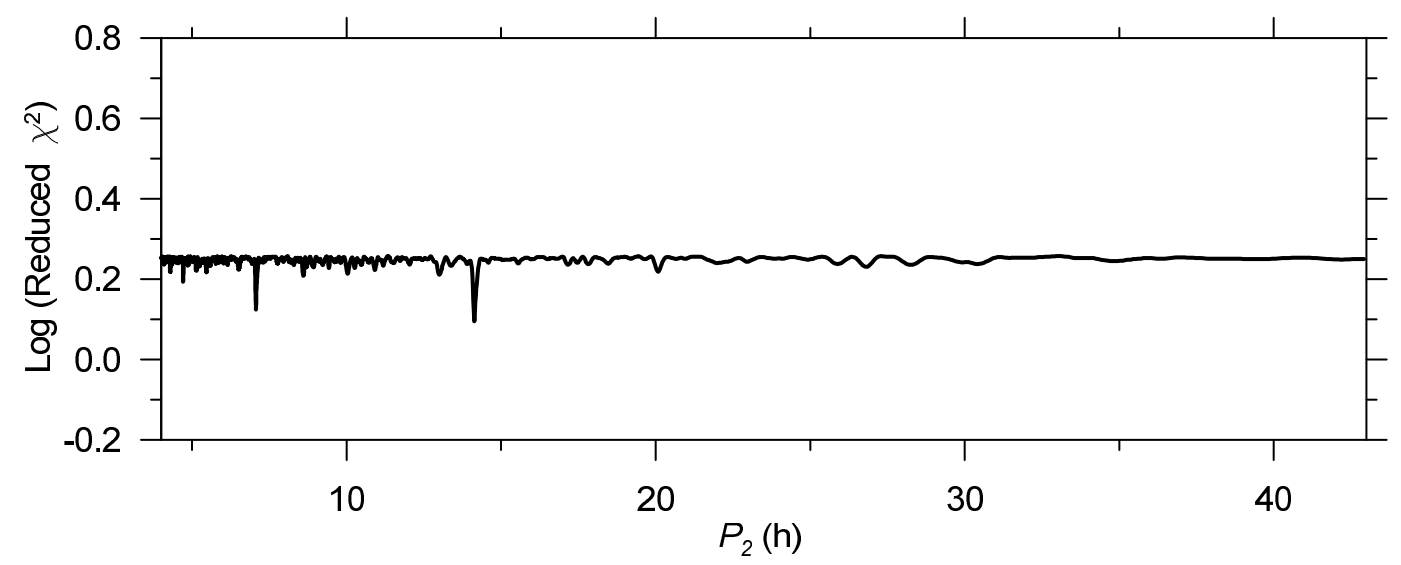

Fig. 23. Noise spectrum for the secondary rotational period of (76818) 2000 RG79, the dataset for Epoch JD 2453609.2215. 


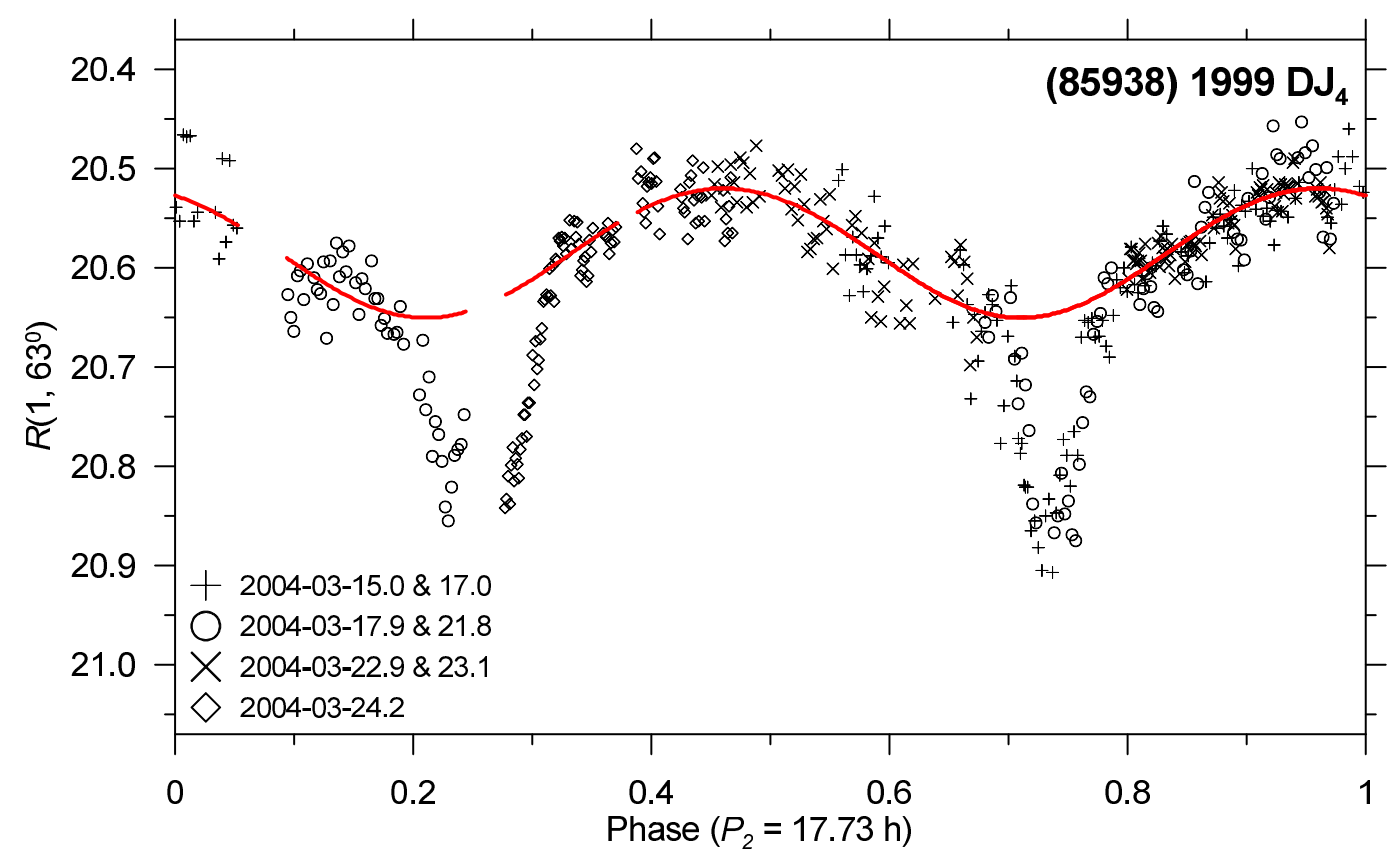

Fig. 24. Secondary lightcurve of (85938) 1999 DJ4.

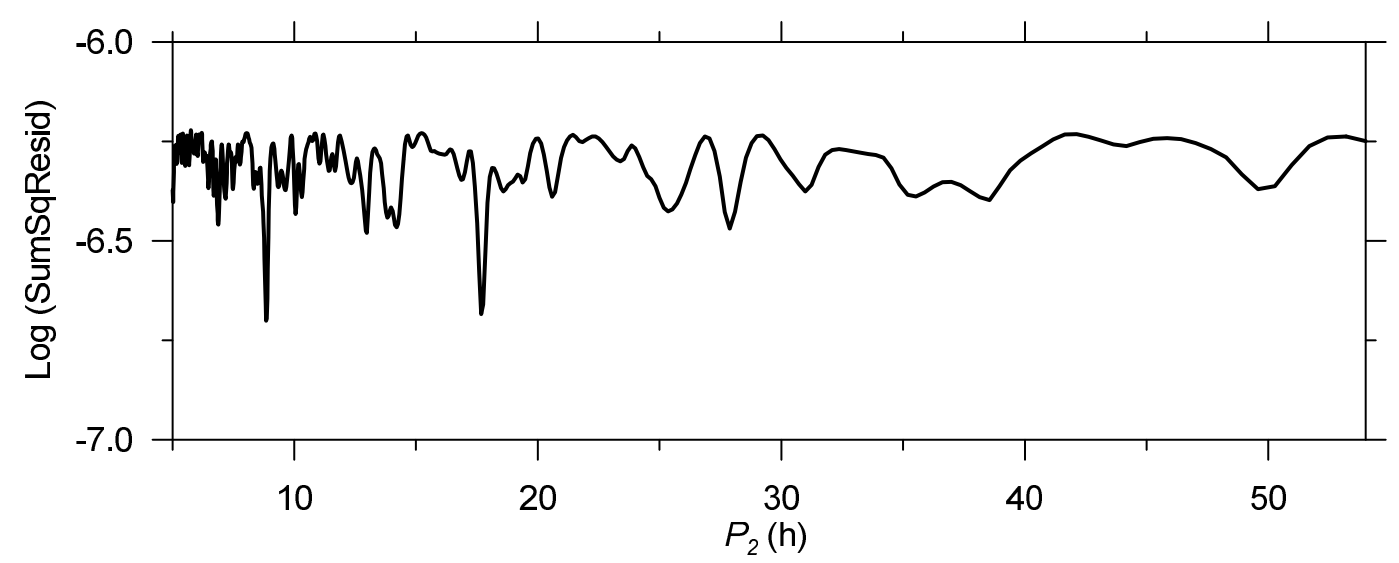

Fig. 25. Noise spectrum for the secondary rotational period of (85938) 1999 DJ4. 


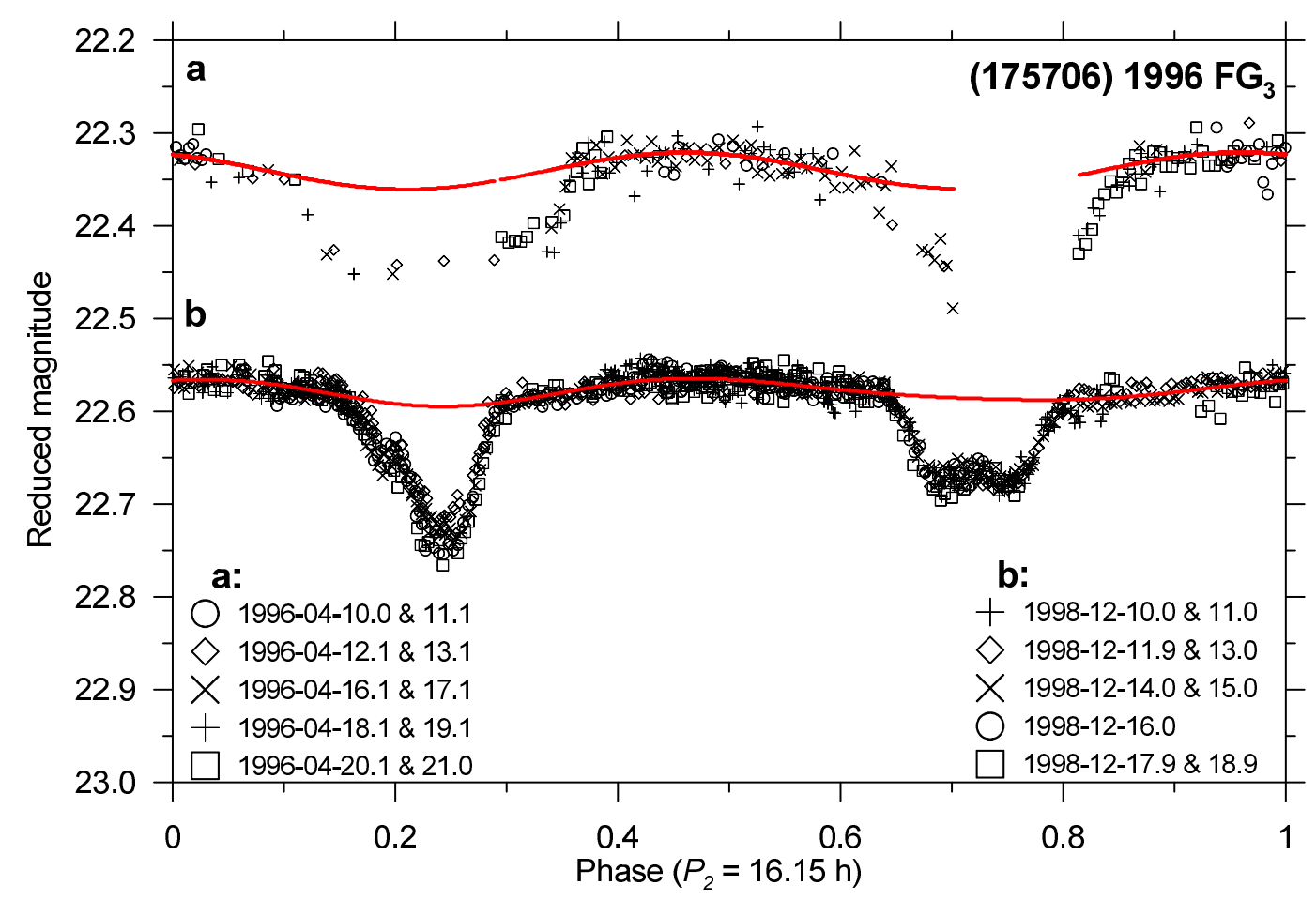

Fig. 26. Secondary lightcurves of (175706) 1996 FG3 from the 1996 and 1998 apparitions. 


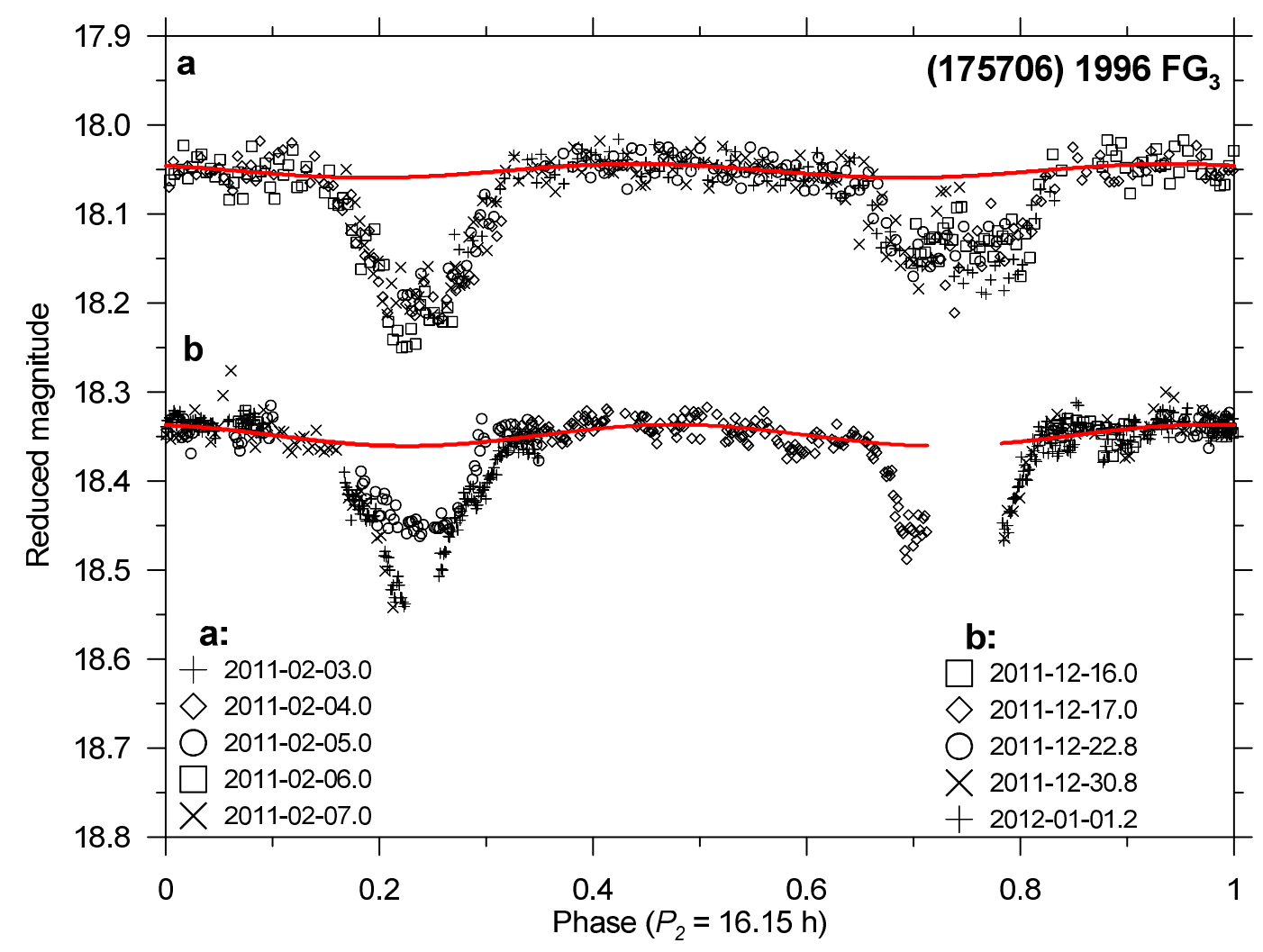

Fig. 27. Secondary lightcurves of (175706) 1996 FG3 from the 2011-2012 apparition.

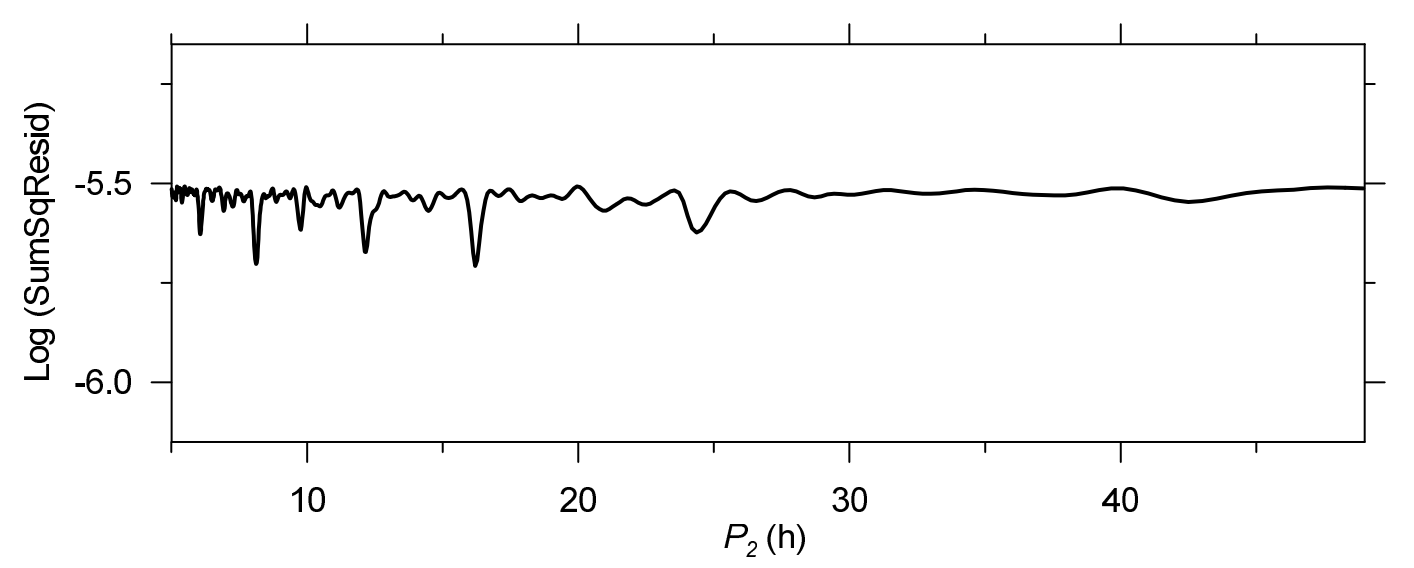

Fig. 28. Noise spectrum for the secondary rotational period of (175706) 1996 FG3, the dataset for Epoch JD 2451161.84583. 


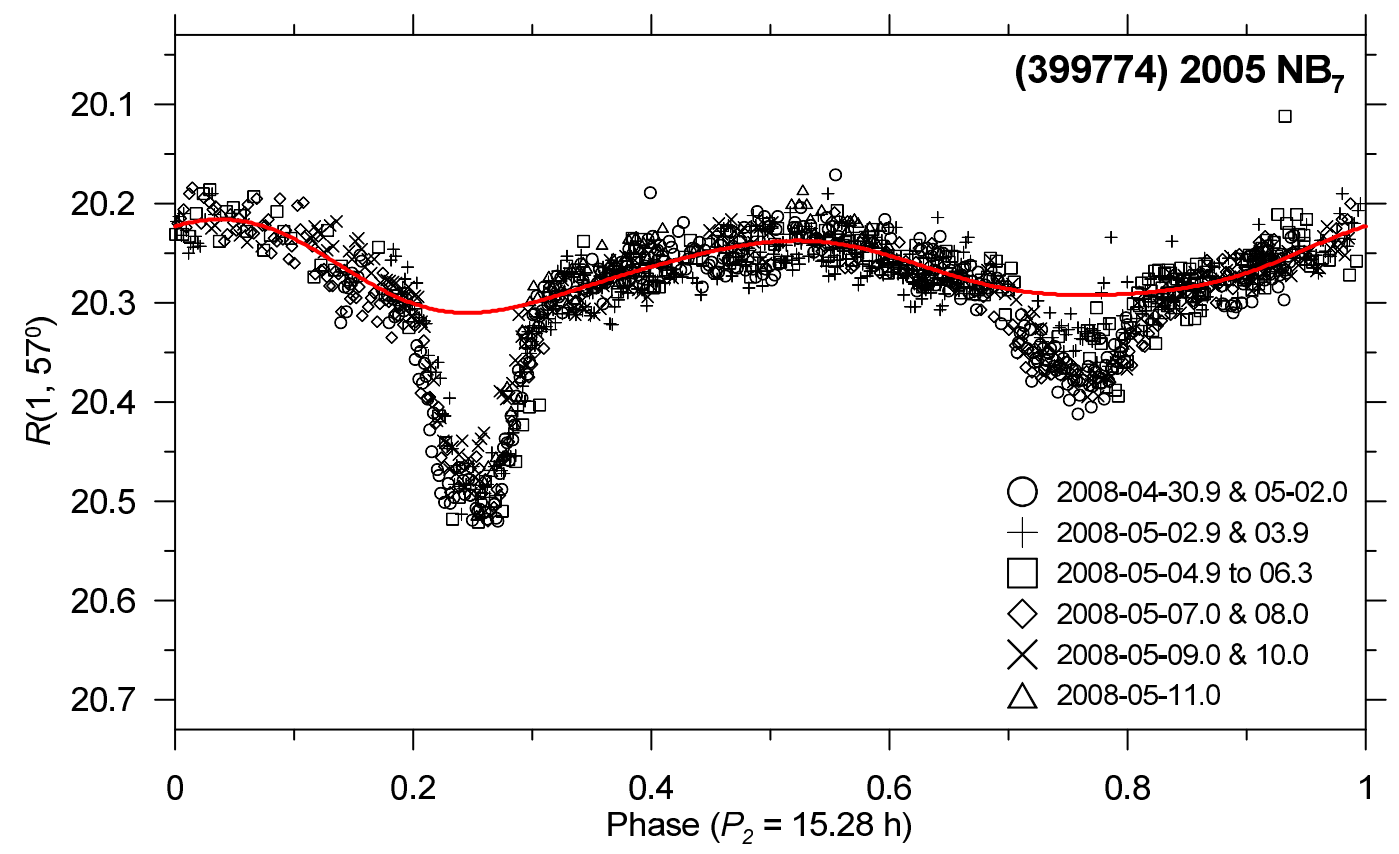

Fig. 29. Secondary lightcurve of (399774) 2005 NB7.

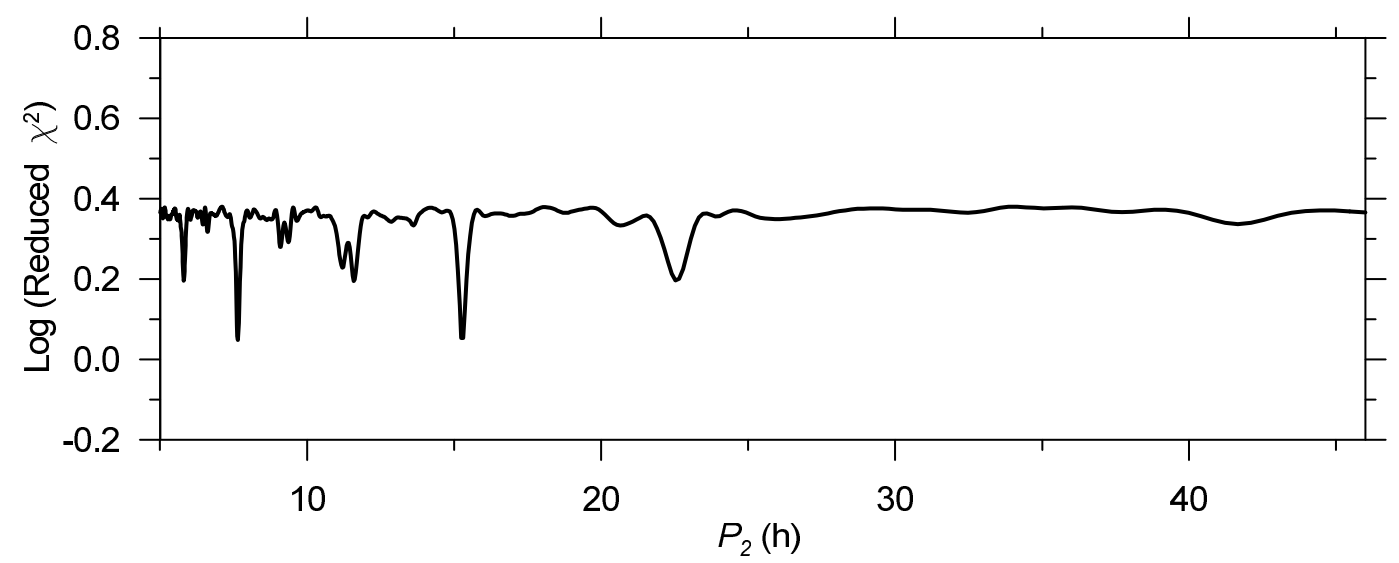

Fig. 30. Noise spectrum for the secondary rotational period of (399774) 2005 NB7. 


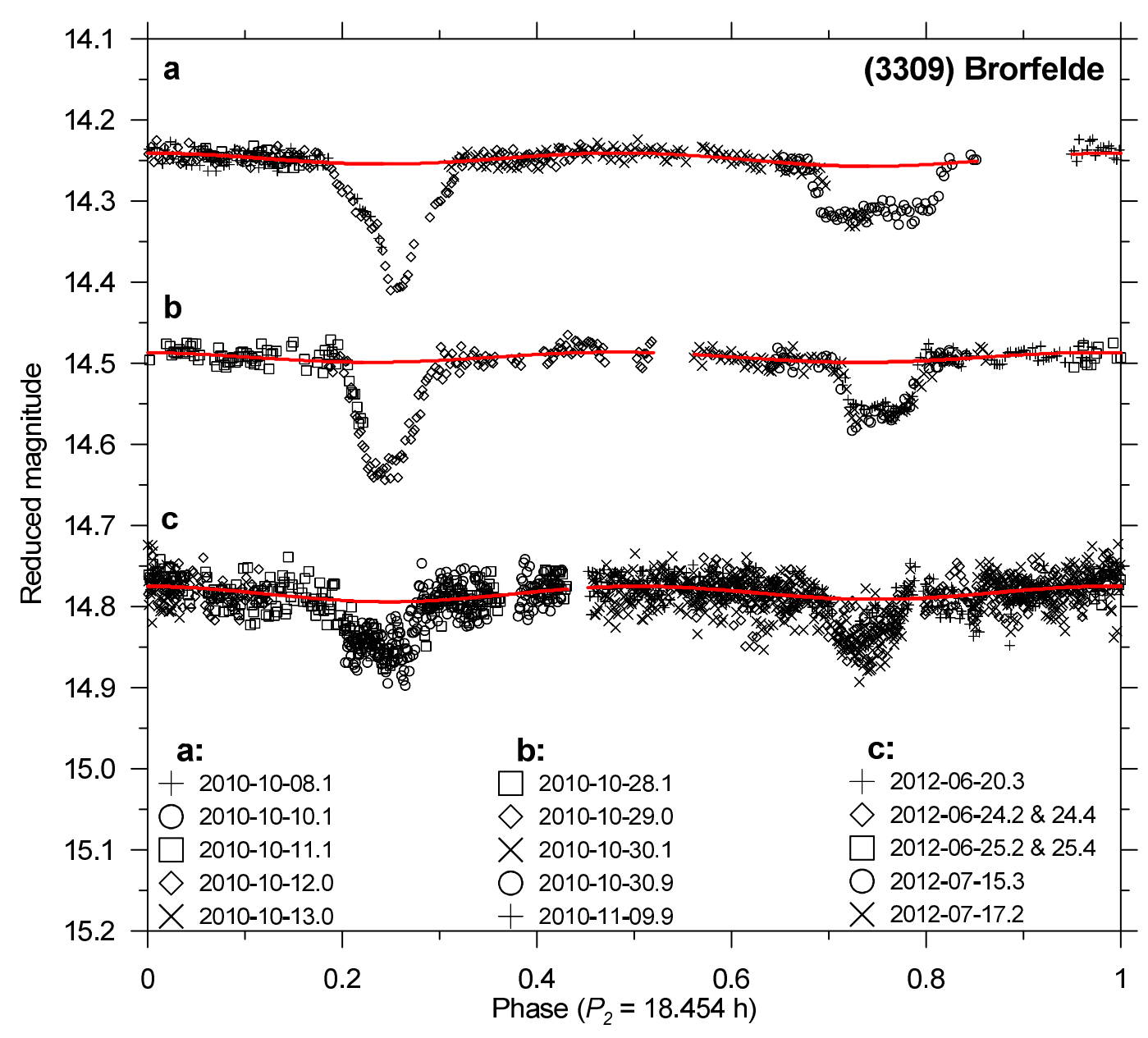

Fig. 31. Secondary lightcurves of (3309) Brorfelde.



Fig. 32. Noise spectrum for the secondary rotational period of (3309) Brorfelde, the dataset for Epoch JD 2455479.78694. 


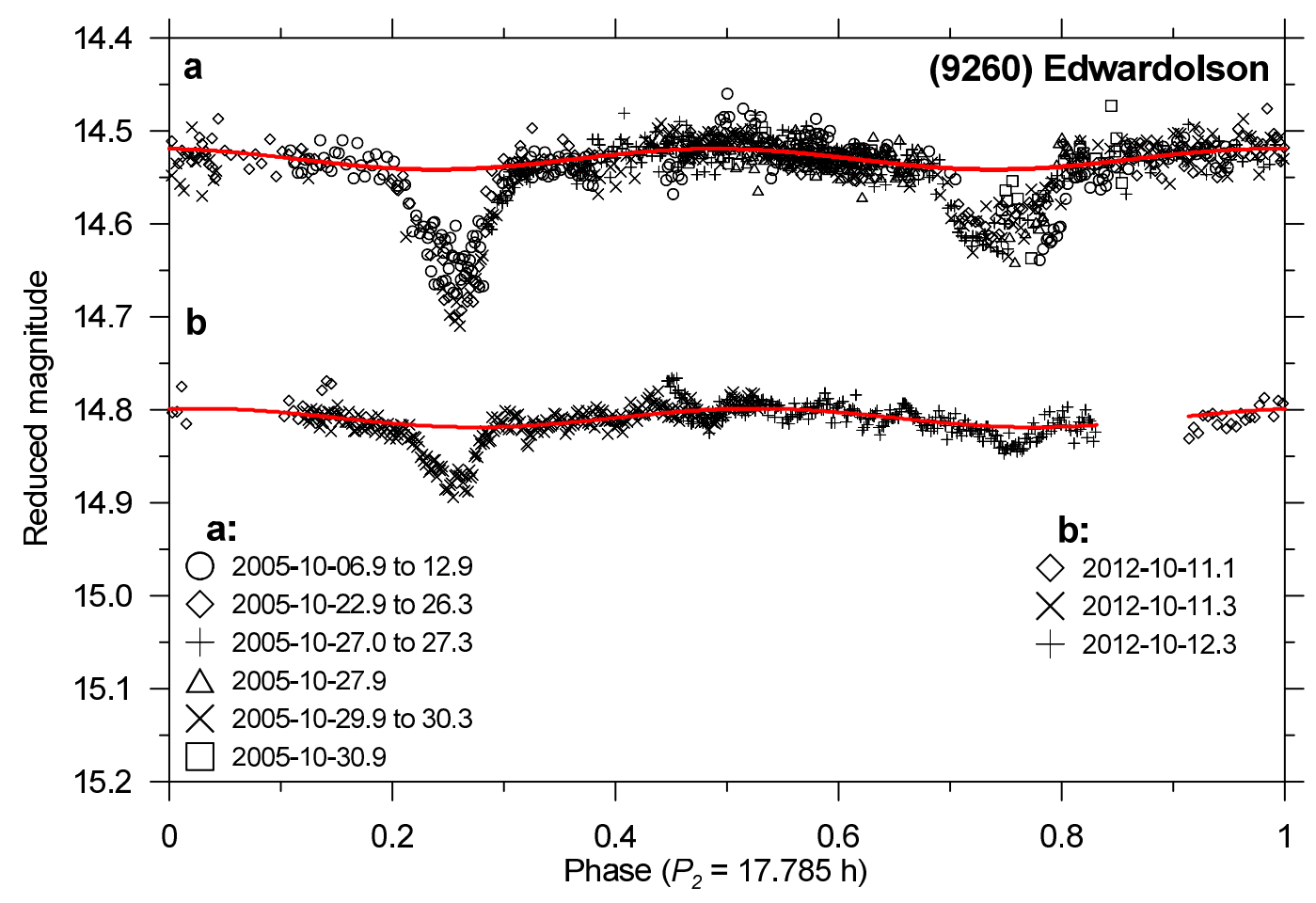

Fig. 33. Secondary lightcurves of (9260) Edwardolson.

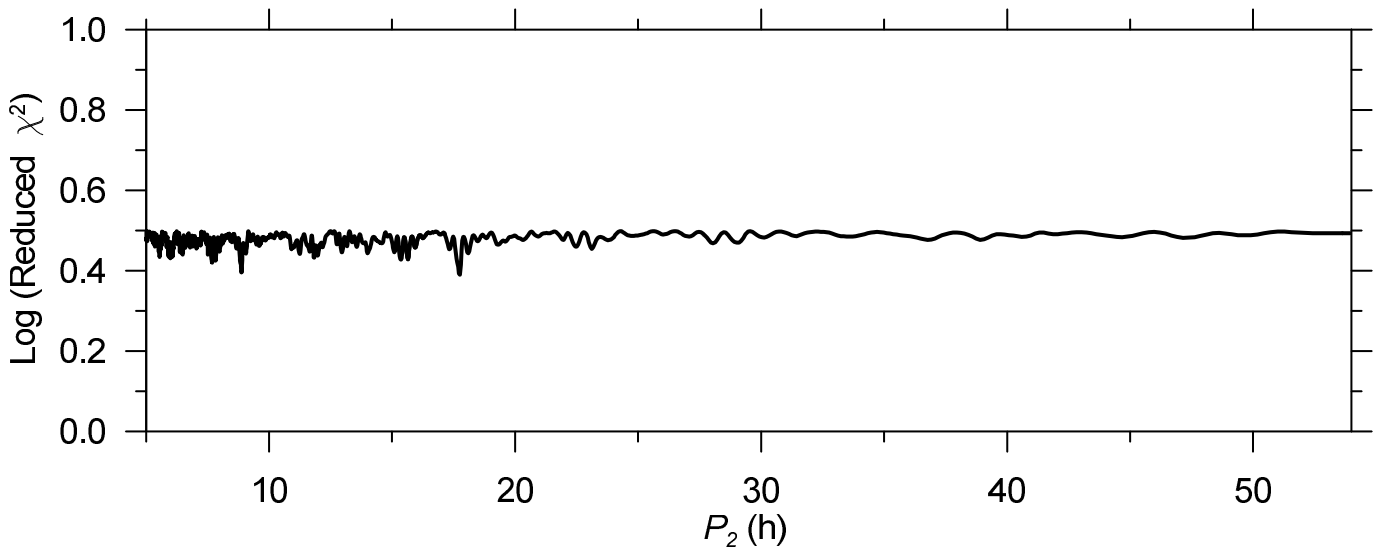

Fig. 34. Noise spectrum for the secondary rotational period of (9260) Edwardolson, the dataset for Epoch JD 2453662.775. 


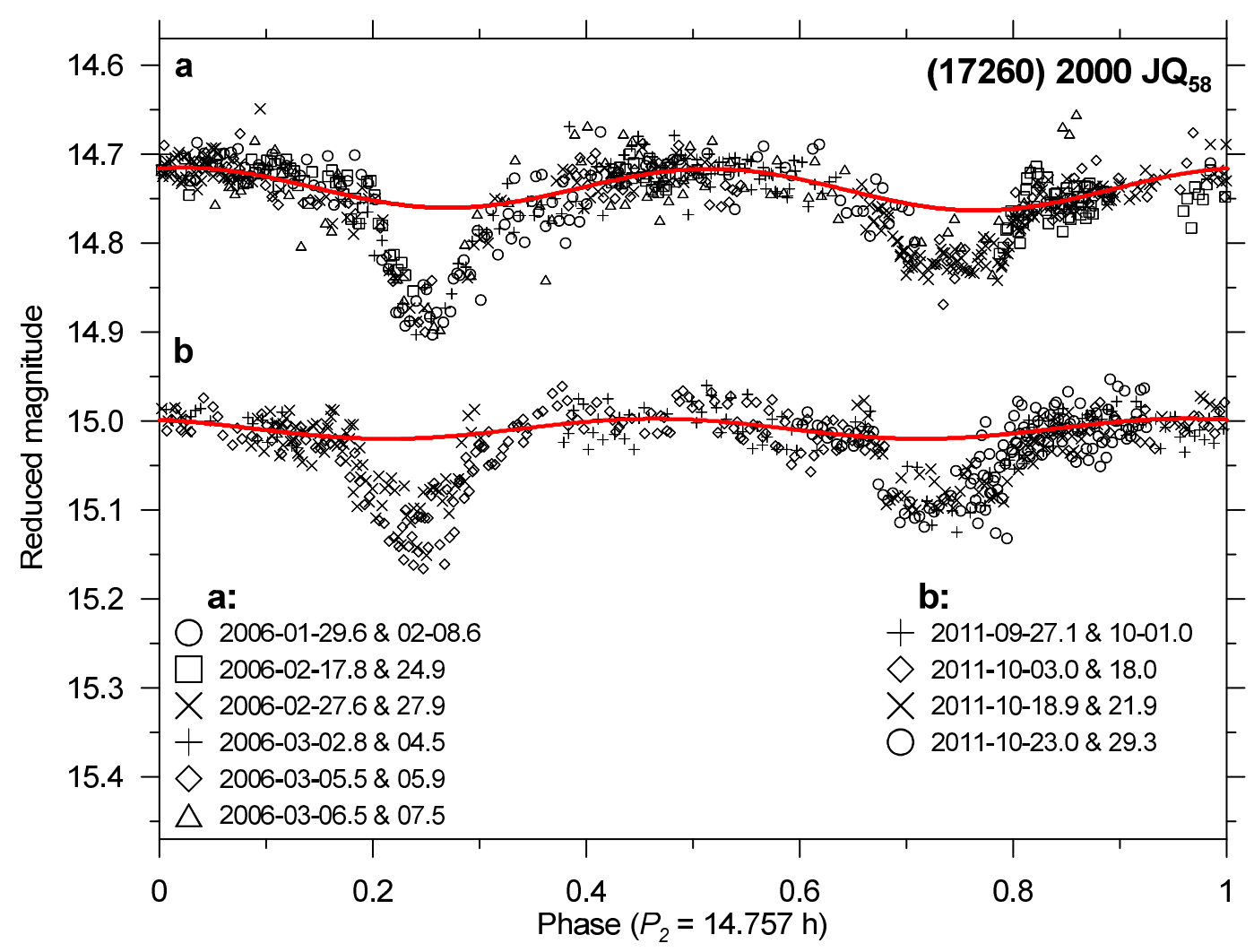

Fig. 35. Secondary lightcurves of (17260) 2000 JQ58.

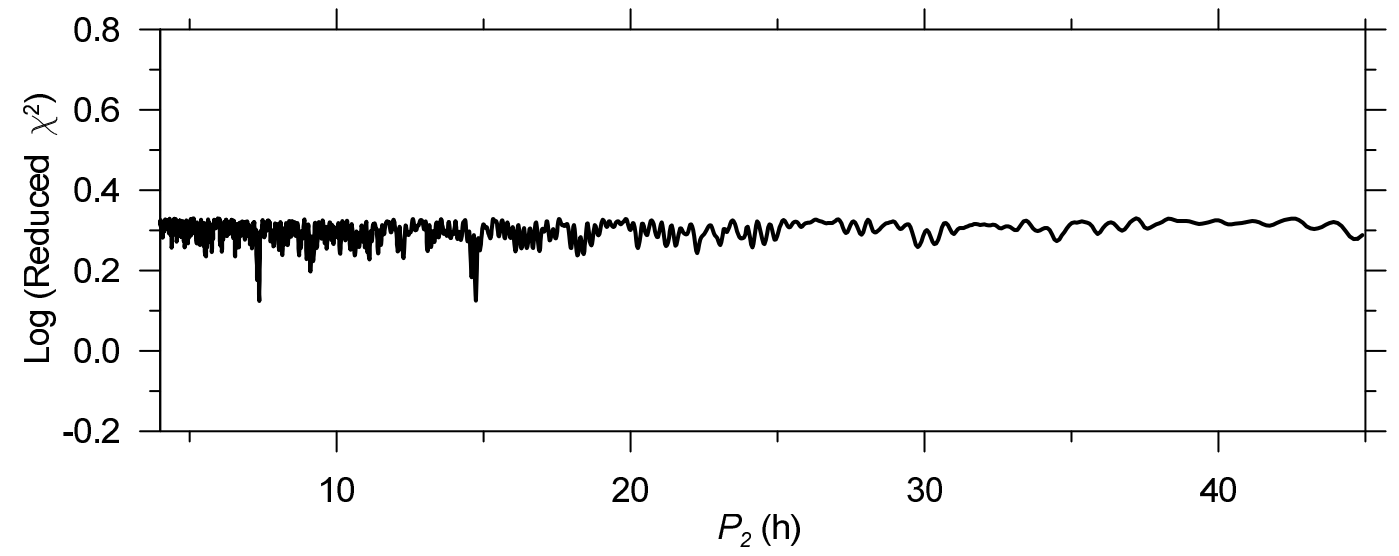

Fig. 36. Noise spectrum for the secondary rotational period of (17260) 2000 JQ58, the dataset for Epoch JD 2453783.42375. 


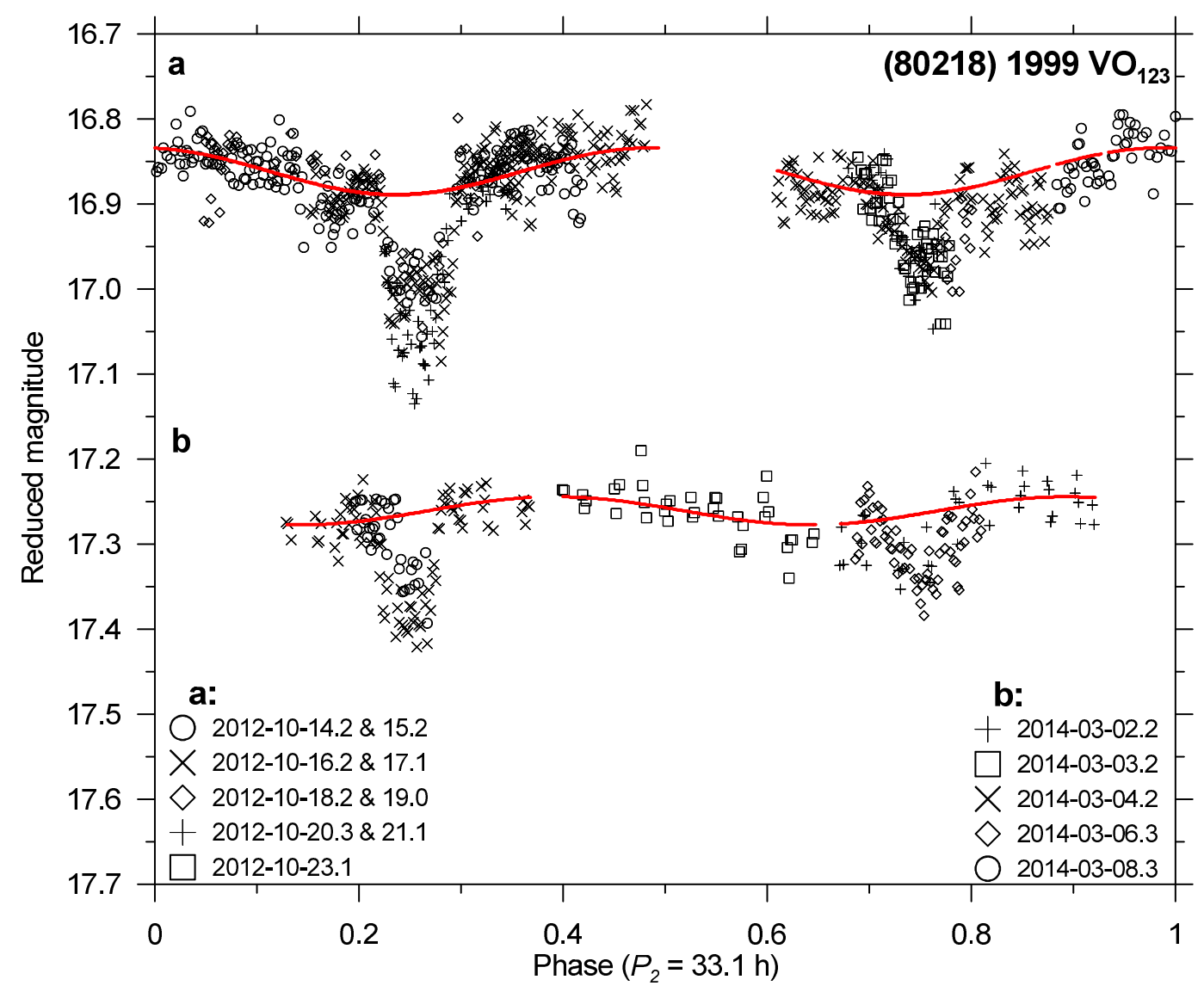

Fig. 37. Secondary lightcurves of (80218) 1999 VO123.

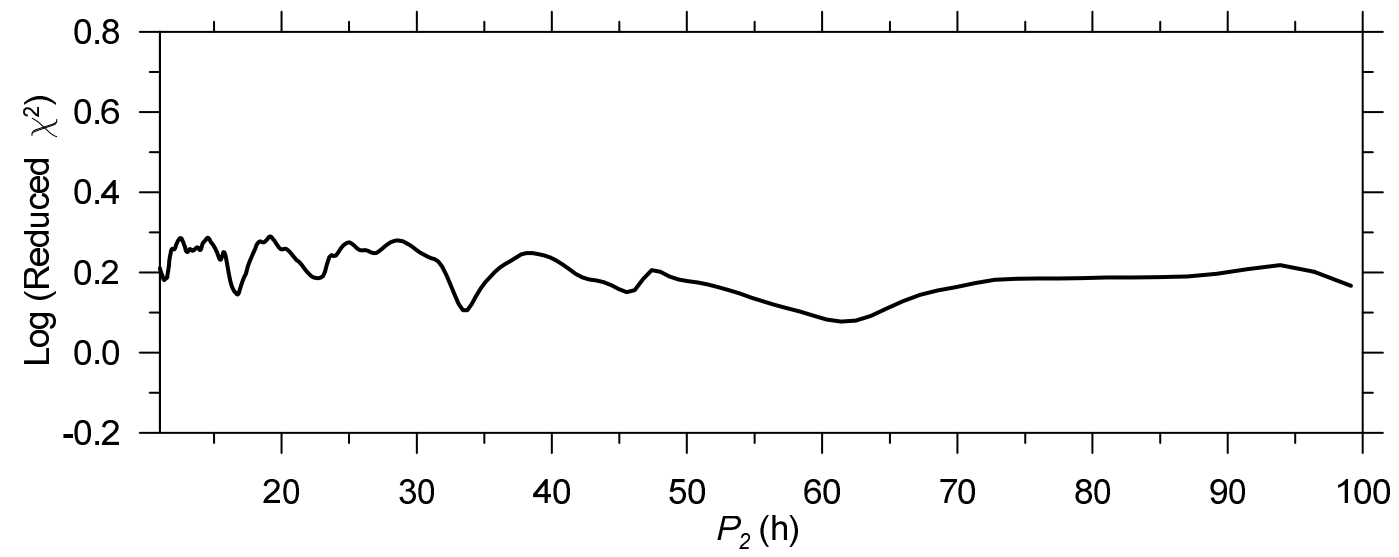

Fig. 38. Noise spectrum for the secondary rotational period of (80218) 1999 VO123, the dataset for Epoch JD 2456218.42241. 


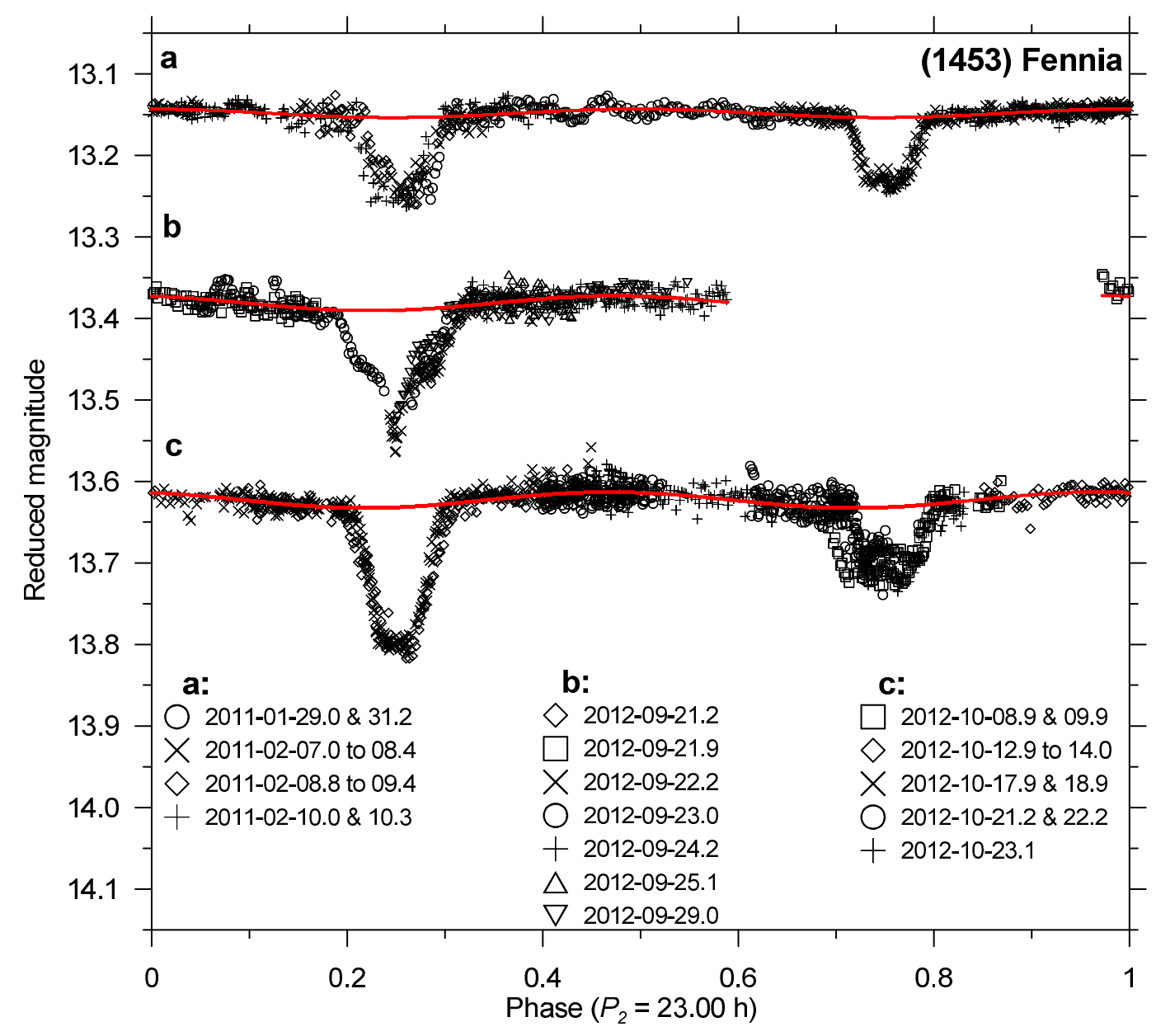

Fig. 39. Secondary lightcurves of (1453) Fennia. 


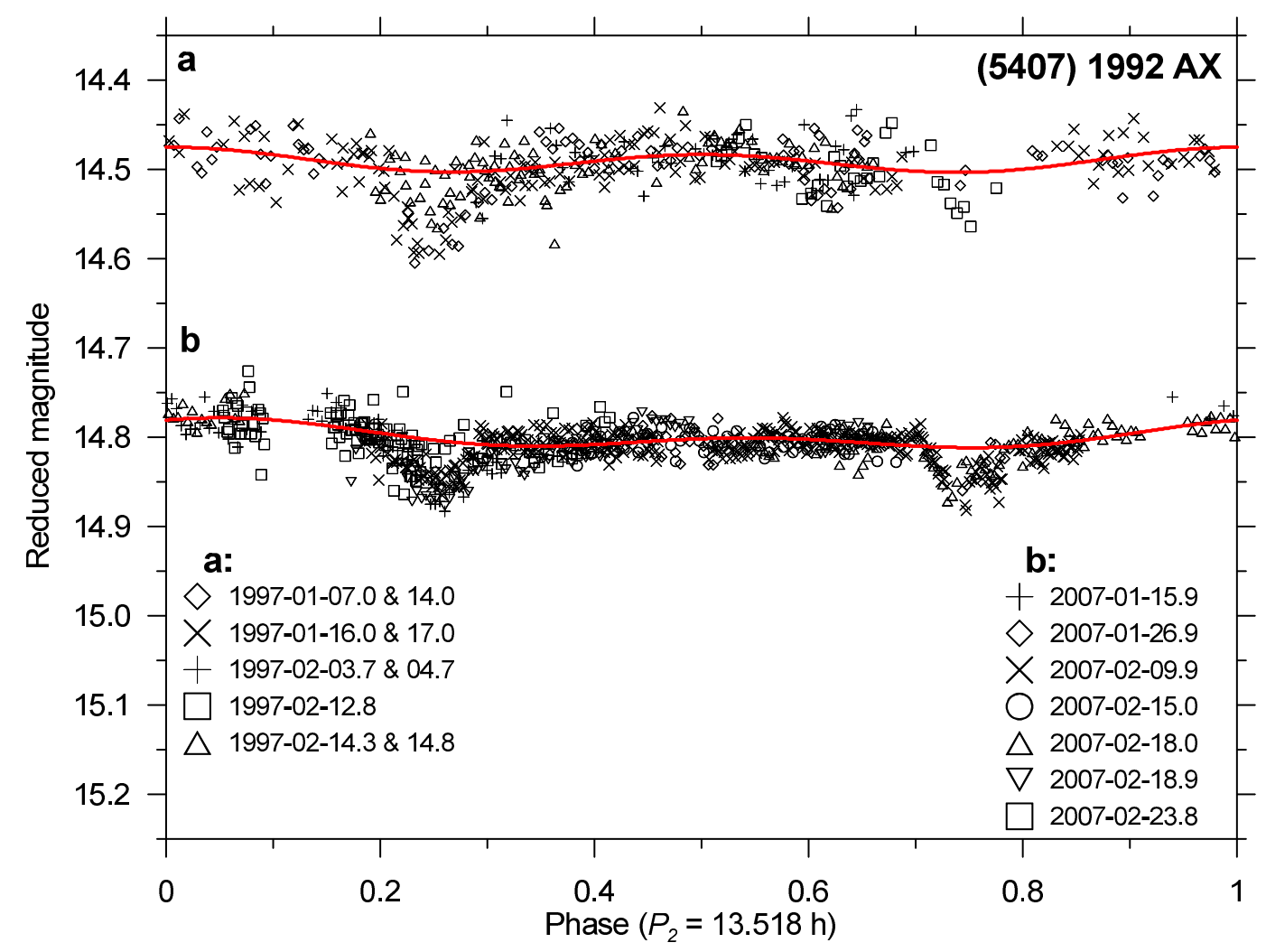

Fig. 40. Secondary lightcurves of (5407) 1992 AX from the 1997 and 2007 apparitions. 


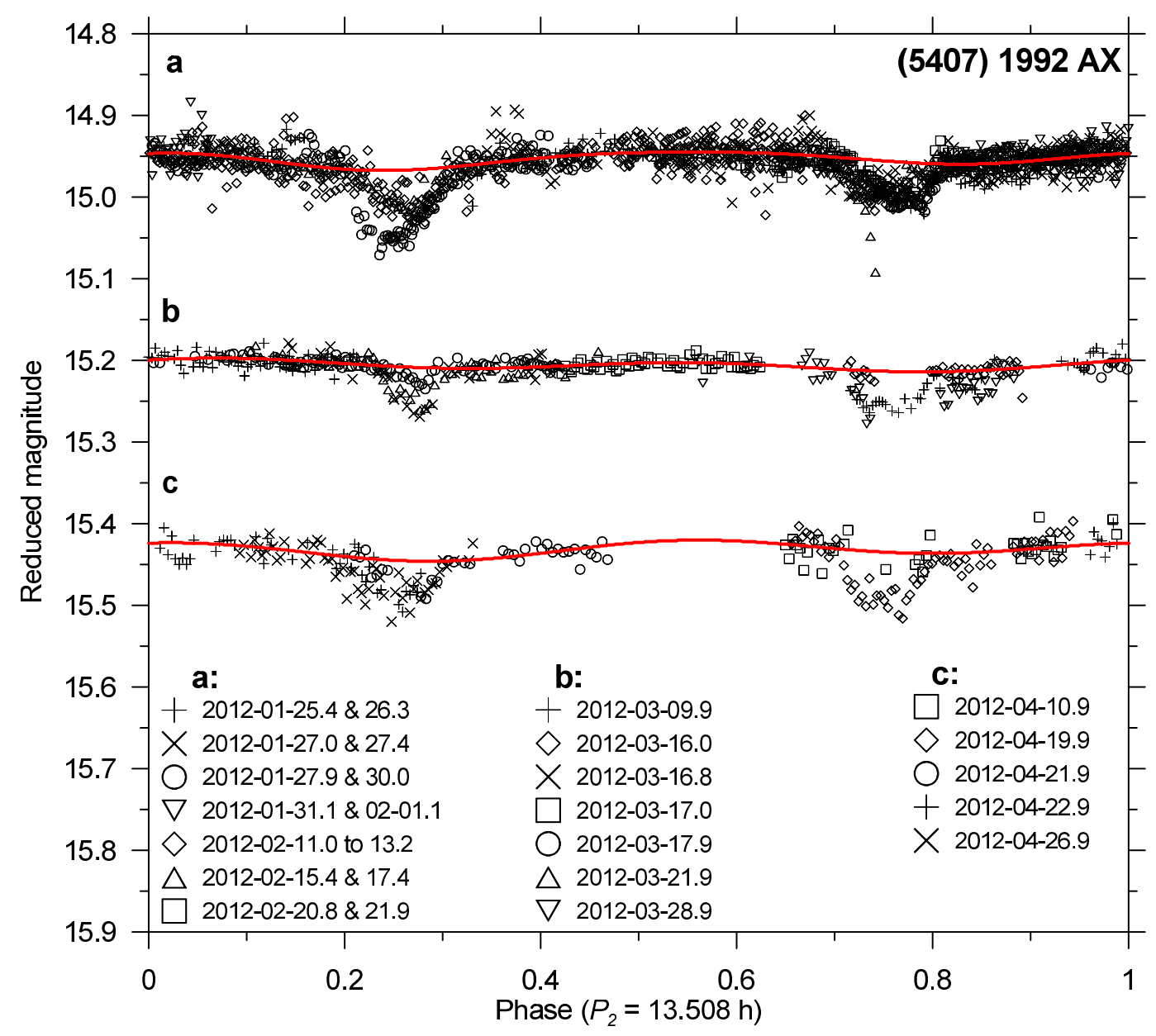

Fig. 41. Secondary lightcurves of (5407) 1992 AX from the 2012 apparition. 


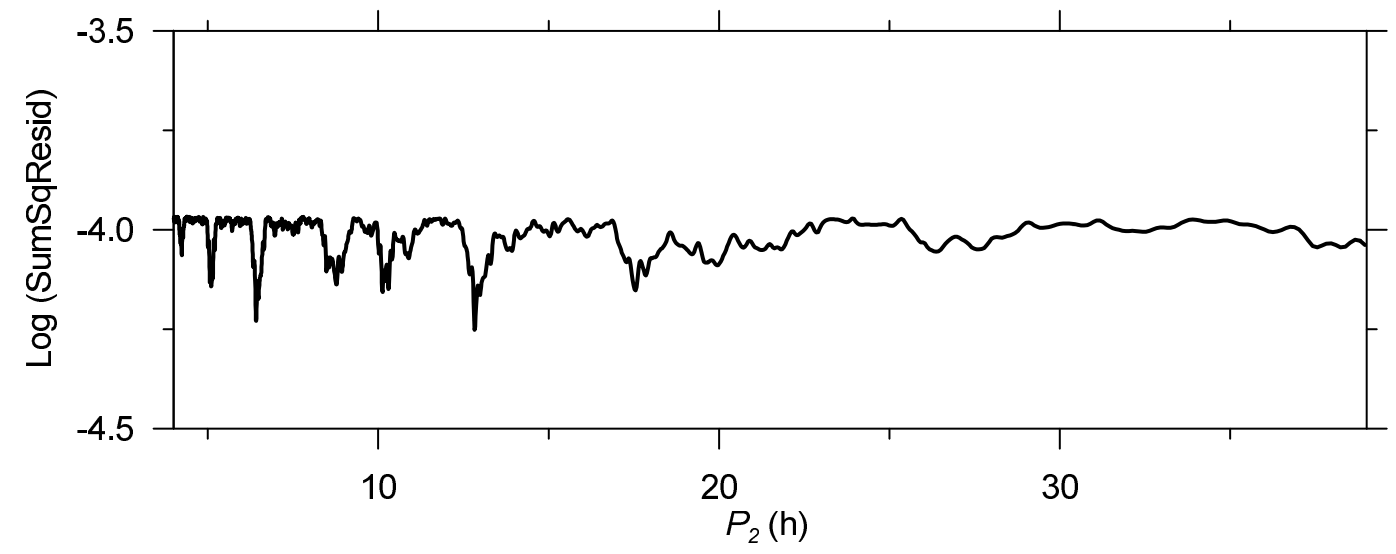

Fig. 42. Noise spectrum for the secondary rotational period of (35107) $1991 \mathrm{VH}$. 


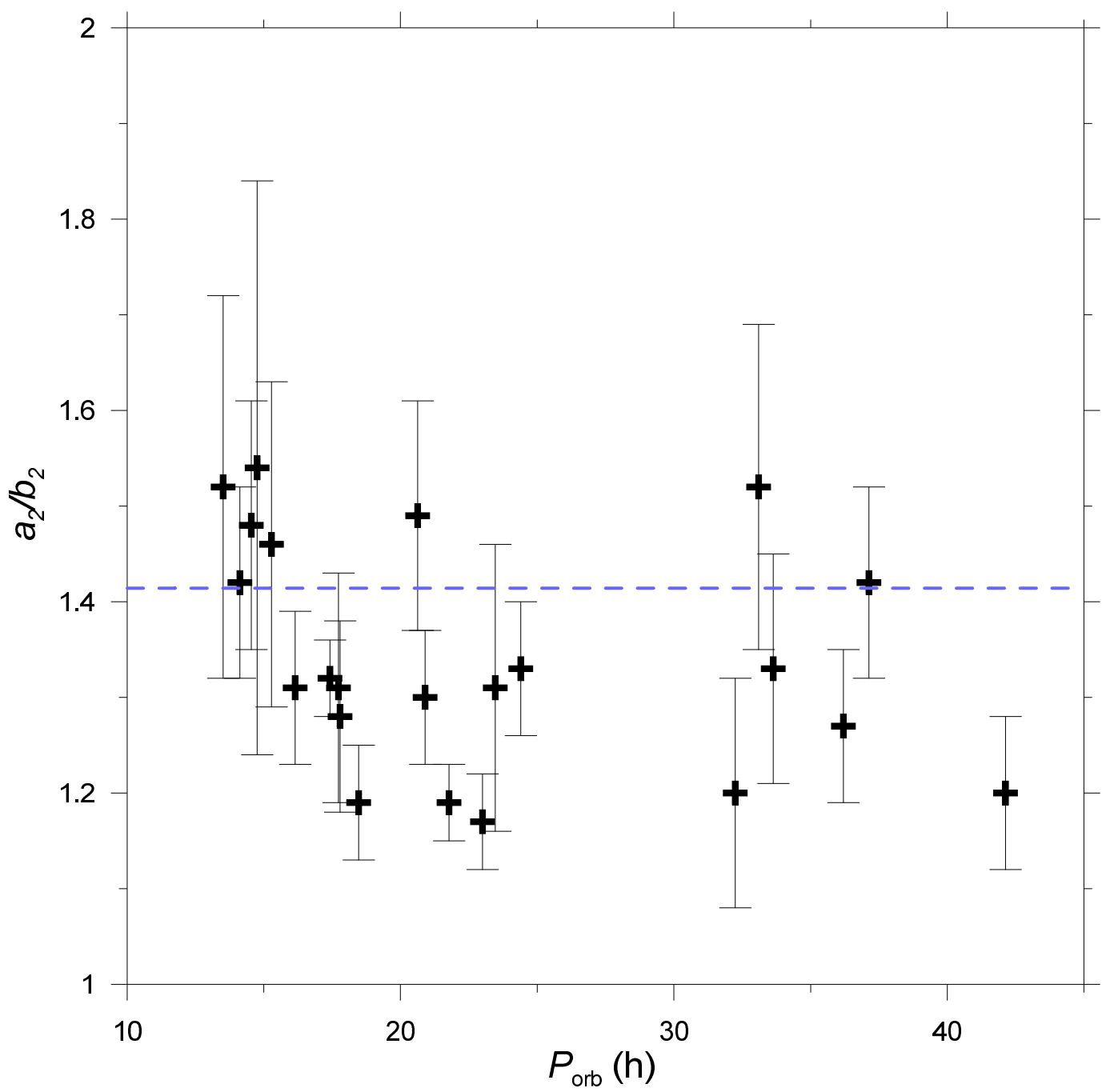

Fig. 43. Equatorial axis ratios vs orbital periods for the observed synchronous secondaries. The dashed line is $a_{2} / b_{2}=\sqrt{2}$, see text. 